\title{
Computing Equilibria in Finance Economies with Incomplete Markets and Transaction Costs*
}

\author{
P. Jean-Jacques Herings ${ }^{\dagger} \quad$ Karl Schmedders ${ }^{\ddagger}$
}

June 12, 2001

\begin{abstract}
Transaction costs in financial markets may have important consequences for volumes of trade, asset pricing and welfare. In the economic literature they are often given as one reason for the incompleteness of asset markets, which is a striking example of their potential impact on volumes of trade. We argue that analytical results on the impact of transaction costs are hard to obtain and a computational approach is needed. This paper introduces the first algorithm for the computation of equilibria in the general equilibrium model with incomplete asset markets and linear transaction costs on the financial markets. The algorithm is based on the homotopy principle and is able to deal with the two major technical difficulties of the model, namely the existence of non-differentiabilities of agents' asset demands as a function of the asset prices and the existence of locally non-unique equilibria. Several numerical examples are used to illustrate the algorithm. We find that the comparative statics of transaction costs for volumes of trade and prices can be rather complicated even for very simple examples of small economic models.
\end{abstract}

\footnotetext{
${ }^{*}$ This research started when Jean-Jacques Herings enjoyed the generous hospitality of the Cowles Foundation for Research in Economics at Yale University. His research has been made possible by a fellowship of the Royal Netherlands Academy of Arts and Sciences. The authors thank audiences at Stanford University, UC San Diego, and Venice for helpful comments.

${ }^{\dagger}$ Department of Economics, Universiteit Maastricht, P.O. Box 616, 6200 MD Maastricht, The Netherlands. Homepage: http://www.fdewb.unimaas.nl/algec/ E-mail: P.Herings@algec.unimaas.nl

${ }^{\ddagger}$ Department of Managerial Economics and Decision Sciences, Kellogg Graduate School of Management, Northwestern University, Evanston, IL 60208, USA. E-mail: k-schmedders@kellogg.nwu.edu
} 


\section{Introduction}

General equilibrium models with incomplete asset markets such as the two-period GEI model and the multi-period model of a stochastic finance economy are generalizations of the classical Arrow-Debreu model of complete markets. While the Arrow-Debreu model considers only spot markets for commodities, the incomplete markets models distinguish between spot markets for commodities and financial markets for securities. As a result these models study many phenomena that the Arrow-Debreu model cannot describe, and they have led to fundamental insights into market behavior. Financial instruments and money significantly influence equilibrium allocations, markets are not even constrained efficient despite being perfectly competitive, and markets may be vulnerable to sunspots. The surveys by Geanakoplos (1990) and Magill and Shafer (1991) discuss other consequences of the incompleteness of markets such as the limitations of representative agent and single commodity models, the conflicting objectives of firms, and the important distinction between nominal and real assets.

However, the GEI model and its multi-period extensions do not attempt to model the reasons for market incompleteness. Instead, these models take the nonexistence of many markets as given; that is, the market structure is exogenous. Geanakoplos (1990) calls the problem of explaining which markets are open and which are closed, "perhaps the most unexplored part of the GEI Model." One of the main arguments for the consideration of incomplete markets is the existence of transaction costs. It is commonly thought that for some markets the set-up or transaction costs are prohibitively large and so these markets are closed. But the exact impact of transaction costs on the level of transactions on the asset markets is not described in these models. This deficiency of the existing models provides us with the motivation to incorporate transaction costs in a GEI model in order to study endogenously determined market incompleteness. We explain below why analytical results on the impact of transaction costs are hard to obtain and a computational approach is needed. This paper introduces the first algorithm for the computation of equilibria in the general equilibrium model with incomplete asset markets and linear transaction costs on the financial markets.

There are several reasons to study transaction costs. Transaction costs are likely to have a big impact on volumes of trade, asset pricing and agents' welfare. The explanation of market incompleteness as a consequence of transaction costs is one striking example of the consequences of transaction costs on the volume of trade of financial assets. Tobin's proposal to impose a tiny tax on asset trades, which plays the role of a grain of sand in the financial system to avoid excessive speculation, is another one. The intuition is in both cases that increasing transaction costs should lead to decreasing volumes of trade. This intuition is confirmed by Constantinides (1986) who finds in an intertemporal consumption and investment model that transaction costs have a first-order effect and reduce the average demand for assets over time substantially.

Transaction costs are likely to have consequences for asset pricing and are sometimes raised as an explanation for the equity premium puzzle. The effect of transaction costs on pricing are at least threefold as has been argued in Heaton and Lucas (1996). There are the two contrary direct effects 
that a seller of an asset desires a higher price and a buyer a lower price when faced with transaction costs. A third, indirect, effect arises as transaction costs make it more costly to change the initial income stream and to insure against bad shocks. This effect makes markets more incomplete and, under certain reasonable assumptions on utility functions, makes agents less willing to trade risky assets, and leads to lower prices for risky assets. A similar reasoning has been used in Elul (1997) to show that increasing market incompleteness, and therefore increasing transaction costs, leads to a lower riskfree rate and a higher price for a riskless asset. A priori it is unclear which of the effects, the indirect one or the direct ones, dominate.

Analytic results are almost impossible to obtain when transaction costs prevail in a GEI economy, unless when one is willing to make very strong assumptions like mean-variance preferences. But even then such results provide little insight as they would be characterized by a huge number of different regimes that depend on the individual bid-ask structures in the economy. Transaction costs lead to non-differentiable comparative statics with a non-differentiability occurring each time an individual trader changes the sign of his position in an asset market; that is, whenever he reduces a short position or a long position down to zero, or does the opposite. These non-differentiabilities greatly obscure the potential insights from analytical results. This difficulty explains why comparative static results for GEI economies with transaction costs are not available in the literature to the best of our knowledge.

We propose a computational approach instead, thereby extending the work of Brown, DeMarzo and Eaves (1996a, 1996b), DeMarzo and Eaves (1996), and Schmedders (1998, 1999) on the computation of GEI equilibria to a setting with transaction costs. The non-differentiabilities mentioned above imply that such an extension is far from being straightforward. Moreover, it will turn out that due to transaction costs robust examples of locally non-unique equilibria exist, which is a fact that complicates matters further.

The purpose of this paper is to lay the theoretical foundation for a computational analysis of the impact of transaction costs in GEI economies. The main contribution of this paper is to present the first algorithm for the computation of equilibria in finance economies with transaction costs. The algorithm is based on the homotopy principle. Finding equilibria in models with transaction costs is frequently thought of as a combinatorial problem (see Duffie and Jackson (1989)) which leads to the conclusion that the search for an equilibrium is quite cumbersome. We show that instead we can phrase the problem as solving a single, small, system of equations. Our algorithm allows a fast and reliable computation of equilibria.

The theoretical development of our algorithm reveals difficulties that are of economic importance. Excess demand functions are typically not differentiable and equilibria do not need to be locally unique. In particular, equilibria with closed markets typically exhibit a continuum of asset prices in the closed market. This fundamental difference between models with and without transaction costs implies that computing equilibria in transaction cost models is not a simple extension of the known methods for models without transaction costs. The way we take care of these problems are of general interest and not restricted to the computation of equilibria in the GEI economies with transaction costs. 
The technical approach of the paper is presented in a novel way that, we believe, exemplifies how a homotopy approach should be motivated, validated, and implemented. We present three practically identical homotopies, each of which has its own advantages depending on what we want to accomplish. The first one, the intuitive homotopy, is most useful for an intuitive description of the conceptual approach and the ensuing difficulties. The second one, the theoretical homotopy, is used for the proofs and the precise description of the algorithm. Finally, the third one, the practical homotopy, is the easiest for a robust implementation of our algorithm.

We develop our algorithm for a finance economy, that is, an economy with a single consumption good. This restriction does not seriously limit the implications of our work. First, with transaction cost the usual drop-in-rank problem does not lead to discontinuities in the demand functions and the resulting existence problems. Secondly, restricting attention to a one-good model allows us to better highlight the serious difficulties caused by transaction costs, namely kinks in the demand functions and robustness of local non-uniqueness of equilibria. And finally, finance economies are very popular in the literature and have seen many applications.

The remainder of the paper is organized as follows. In Section 2 we describe the model of a finance economy with transaction costs and characterize the set of no-arbitrage prices. Section 3 introduces the intuitive homotopy and outlines the main ideas of our homotopy approach. In Section 4 we define the theoretical homotopy which is used for our proofs. Section 5 depicts the homotopy that we use for the implementation of the algorithm, and in Section 6 we report numerical results. The paper concludes with some final remarks in Section 7 and an appendix containing the proofs of all results.

\section{A Finance Economy with Transaction Costs}

We consider the standard model of a finance economy with the additional feature of transaction costs on the financial markets. There are two dates, $t=0,1$, with uncertainty at $t=0$ about the state of nature that realizes at $t=1$. We identify date 0 with state of nature 0 . At date 1 , exactly one out of $S$ possible states of nature realizes.

In the economy there are $H$ agents. An agent $h$ is characterized by his initial income stream $e^{h} \in \mathbb{R}^{1+S}$ and his preferences. The future initial income stream, which is uncertain, is denoted by $e_{11}^{h} \in \mathbb{R}^{S}$. Agent $h$ has a preference over income spent for consumption in the various states, $c^{h} \in \mathbb{R}_{+}^{1+S}$. Preferences of agent $h$ are represented by a utility function $u^{h}: \mathbb{R}_{+}^{1+S} \rightarrow \mathbb{R}$.

Agents have the possibility to use $J$ assets in order to modify their income stream across time and across states. Asset $j$ pays a dividend $d_{s}^{j}$ in state of nature $s \in S$. The stream of dividends is $d^{j}=\left(d_{1}^{j}, \ldots, d_{S}^{j}\right)^{\top}$ and the asset return matrix is $A=\left(d^{1}, \ldots, d^{J}\right)$. Without loss of generality, assets are in zero net supply. Indeed, a model where agent $h$ has an initial income stream $\widetilde{e}^{h}$ and an initial asset portfolio $\widetilde{\theta}^{h}$ is equivalent to a model where agent $h$ has zero initial holdings of all assets and an initial income stream $e^{h}$ given by $e_{0}^{h}=\widetilde{e}_{0}^{h}$ and $e_{\mathbb{1}}^{h}=\widetilde{e}_{\mathbb{1}}^{h}+A \widetilde{\theta}^{h}$. With a slight abuse of notation we also use $H, J$ and $S$ to denote the set of agents, the set of assets and the set of future states of nature, respectively. 
Prices $q=\left(q_{1}, \ldots, q_{J}\right)^{\top}$ of assets are denoted in units of income. Sales of assets by agent $h$ are denoted by $\theta^{h,-} \in \mathbb{R}_{+}^{J}$ and purchases by $\theta^{h,+} \in \mathbb{R}_{+}^{J}$. The net trade in assets then leads to an asset portfolio $\theta^{h}=\theta^{h,+}-\theta^{h,-}$. Both buyers and sellers of assets incur real transaction $\operatorname{costs}^{1}$. Agent $h$ 's trade $\left(\theta^{h,-}, \theta^{h,+}\right)$ leads to transaction costs $\sum_{j \in J} k_{j}^{h,-} \theta_{j}^{h,-}+\sum_{j \in J} k_{j}^{h,+} \theta_{j}^{h,+}$. Here, $k_{j}^{h,-}$ and $k_{j}^{h,+}$ are nonnegative transaction costs, denoted in units of income per unit of trade in asset $j$. For notational simplicity, we assume $k_{j}^{h,-}=k_{j}^{h,+}=k_{j}^{h^{\prime},+}$ for all agents $h$ and $h^{\prime}$, and denote these costs by $k_{j}$. This approach to modeling transaction costs is identical to the one in Arrow and Hahn (1999). A finance economy with incomplete markets and transaction costs is given by $\mathcal{E}=\left\{\left(e^{h}, u^{h}\right)_{h \in H}, A, k\right\}$.

Throughout the paper we make the following assumptions.

A1 For all $h \in H$, the initial income stream $e^{h}$ belongs to $\mathbb{R}_{++}^{1+S}$. We define the open set $E=$ $\mathbb{R}_{++}^{(1+S) H}$.

A2 For all $h \in H$, the utility function $u^{h}$ is three times continuously differentiable on $\mathbb{R}_{++}^{1+S}$, $\partial u^{h} \gg 0, \partial^{2} u^{h}$ is negative definite, and interior income streams are preferred to income streams with zero components, $u^{h}\left(c^{h}\right)>u^{h}\left(\bar{c}^{h}\right)$, when $c^{h} \in \mathbb{R}_{++}^{1+S}$ and $\bar{c}^{h} \in \mathbb{R}_{+}^{1+S} \backslash \mathbb{R}_{++}^{1+S}$.

A3 The rank of the asset return matrix $A$ is $J$.

A4 For all $j \in J$, transaction costs are positive, $k_{j}>0$.

With the exception of the requirement that $\partial^{2} u^{h}$ is negative definite everywhere, Assumptions $\mathrm{A} 1$ and $\mathrm{A} 2$ are standard in the literature, when differentiability of demand functions is needed for the analysis. The stronger assumption on $u$ can be made without loss of generality (Mas-Colell (1985)), however, and it simplifies some of our proofs. The assumption that interior income streams are preferred is standard as well, but can easily be dispensed with when using the

\footnotetext{
${ }^{1}$ In the economic theory literature there have been at least three other approaches to the GEI model with transaction costs. Yamazaki (1991) proves existence of an equilibrium for GEI economies with real transaction costs and outside money. Both transactions on the asset markets and on the spot markets incur transaction costs. These transaction costs are expressed in terms of individual transaction possibility sets. Laitenberger (1996) considers a GEI economy with real transaction costs on the financial markets, where transaction costs take the form of a proportional loss of at least one good in the second period. In addition to proving the existence of an equilibrium, Laitenberger shows how the usual no-arbitrage condition must be modified for her model with real transaction costs. Préchac (1994) introduces intermediation costs, that take the form of commissions proportional to the value of trades. The commissions are paid to a privately owned brokerage house that redistributes the commissions to the consumers. These intermediation costs also result in endogenous bounds on the asset transactions and thereby ensure the existence of an equilibrium. Préchac (1994) also develops a new no-arbitrage condition for his model.

In the macroeconomic literature, transaction costs have usually been assumed to be quadratic in the volume of trade, an example is the paper of Heaton and Lucas (1996). This assumption avoids any differentiability problems and the computation of equilibria can be handled by any standard homotopy algorithm. It seems hard to defend, however, that quadratic transaction costs are a reasonable description of transaction costs made in the real world, even if one incorporates increasing bid-ask spreads for large transactions. Moreover, quadratic transaction costs cannot be used to generate endogenous market incompleteness as the marginal costs of trading small quantities are zero and typically there is trade in all assets.
} 
techniques developed in this paper. The assumption that the asset return matrix has full rank, A3, is not without loss of generality in the case with transaction costs, but it greatly simplifies the proofs. The general case remains to be investigated in future research.

At an asset price system $q$ the decision problem of agent $h$ consists of choosing an asset trade $\left(\theta^{h,-}, \theta^{h,+}\right) \in \mathbb{R}_{+}^{J} \times \mathbb{R}_{+}^{J}$ and a compatible consumption pattern. He chooses an element of his budget set,

$$
\begin{gathered}
B^{h}(q)=\left\{\left(\theta^{h,-}, \theta^{h,+}, c^{h}\right) \in \mathbb{R}_{+}^{2 J+1+S} \mid \begin{array}{l}
c_{0}^{h}+\sum_{j \in J}\left(q_{j}+k_{j}\right) \theta_{j}^{h,+} \leq e_{0}^{h}+\sum_{j \in J}\left(q_{j}-k_{j}\right) \theta_{j}^{h,-} \\
\left.c_{\mathbb{1}}^{h} \leq e_{\mathbb{1}}^{h}+A\left(\theta^{h,+}-\theta^{h,-}\right)\right\}
\end{array}\right.
\end{gathered}
$$

that maximizes utility.

We restrict ourselves in this paper to transaction costs on the units of assets traded. Of course, we could instead consider transaction costs on the value of assets traded. Our algorithm can easily be extended to models with such transaction costs. Note also that we consider transaction costs in a broad sense, so they incorporate effort, fees, taxes, or bid-ask spreads.

Some asset prices $q \in \mathbb{R}^{J}$ may lead to arbitrage in which case an agent's decision problem does not have a solution. This fact creates the need to characterize the set of no-arbitrage prices. Because of the presence of transaction costs, the set of no-arbitrage prices gets larger than in the model of a finance economies without transaction costs.

Definition 2.1: A vector $q \in \mathbb{R}^{J}$ is a no-arbitrage asset price system when there does not exist an asset portfolio $\left(\theta^{-}, \theta^{+}\right) \in \mathbb{R}_{+}^{J} \times \mathbb{R}_{+}^{J}$ such that $(q-k) \cdot \theta^{-}-(q+k) \cdot \theta^{+}>0$ and $A\left(\theta^{+}-\theta^{-}\right) \geq 0$, or $(q-k) \cdot \theta^{-}-(q+k) \cdot \theta^{+} \geq 0$ and $A\left(\theta^{+}-\theta^{-}\right)>0 .^{2}$

A vector $q$ admits arbitrage possibilities whenever it is possible to make strictly positive profits in at least one state and nonnegative profits in all states. We have the following characterization of no-arbitrage prices.

Proposition 2.2: A vector $q \in \mathbb{R}^{J}$ is a no-arbitrage asset price system if and only if there is $\pi \in \mathbb{R}_{++}^{S}$ such that $\pi^{\top} A-k \leq q \leq \pi^{\top} A+k$.

Proposition 2.2 does not require $A$ to have full column rank, and it is also true for degenerate asset return matrices $A$. For $k=0$ the theorem reduces to the fundamental theorem on the pricing of financial securities. The set of no-arbitrage prices is denoted by $Q$. It follows directly by Proposition 2.2 that $Q$ is no longer a cone with vertex zero. It is not even necessarily a cone with a non-zero vertex (for a definition, see Debreu (1959)). See Figure 1 for a graphical illustration of how the set of no-arbitrage prices of an economy with transaction costs compares to the no-arbitrage cone of

\footnotetext{
${ }^{2}$ For a vector $x \in \mathbb{R}^{n}$ we define $x>0$ as $x_{i} \geq 0$ for all $i$ and $x_{j}>0$ for at least some $j$.
} 
an economy without transaction costs. Figure 1 corresponds to the case

$$
A=\left[\begin{array}{ll}
1 & 2 \\
4 & 3
\end{array}\right], \quad k=\left(\begin{array}{l}
2 \\
1
\end{array}\right)
$$

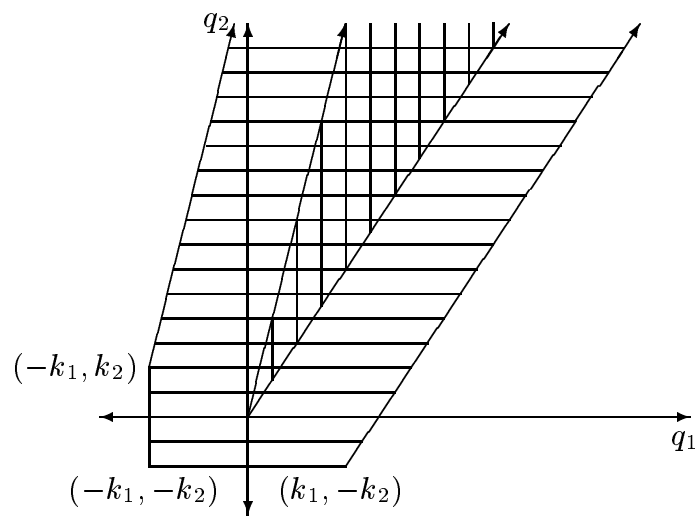

FIGURE 1. The set of no-arbitrage prices.

It is straightforward to show that $Q$ is an open set if $A$ has full column rank.

Proposition 2.3: When $q \in Q$, the budget set $B^{h}(q)$ is compact and convex, and the agent's decision problem has a solution $\left(\theta^{h,-}, \theta^{h,+}, c^{h}\right)$ that is unique and that satisfies $\theta^{h,-} \cdot \theta^{h,+}=0$.

Proposition 2.3 implies that a single agent is never active simultaneously on the demand side and the supply side of an asset market. There is no ambiguity when we do not consider supply and demand of assets separately, but instead use the net asset portfolio purchased, $\theta^{h}=\theta^{h,+}-\theta^{h,-}$. Proposition 2.3 implies that the (net) demand of agent $h$ for assets at prices $q \in Q$ is a function $g^{h}$ : $Q \rightarrow \mathbb{R}^{J}$. The demand of all agents for all assets is given by the $H J$-vector $g(q)=\left(g^{1}(q), \ldots, g^{H}(q)\right)$. Total asset demand is a function $G: Q \rightarrow \mathbb{R}^{J}$, where $G(q)=\sum_{h=1}^{H} g^{h}(q)$. Obviously, the income stream used for consumption is determined uniquely by the asset portfolio purchased. We finish the discussion of the economic model with the definition of a competitive equilibrium.

DeFinition 2.4: A competitive equilibrium for an economy $\mathcal{E}$ is a collection of portfolio holdings $\theta^{*}=\left(\theta^{* 1}, \ldots, \theta^{* H}\right) \in \mathbb{R}^{H J}$ and asset prices $q^{*} \in \mathbb{R}^{J}$ such that

1. $\theta^{* h}$ is a utility maximizing asset portfolio for agent $h$ at prices $q^{*}$,

2. $\sum_{h \in H} \theta^{* h}=0$.

The price vector $q^{*}$ is a competitive equilibrium price system if and only if $G\left(q^{*}\right)=0$. Equilibrium asset portfolios and equilibrium incomes spent on consumption in each state are completely determined by equilibrium prices, since, due to the monotonicity of the utility function, the solution to an agent's optimization problem satisfies all inequalities in the definition of the budget set with 
equality ${ }^{3}$.

At this point we can give a preview of the difficulties in this model to compute equilibria which are due to the transaction costs. Neither the individual asset demand functions $g^{h}$ nor the total asset demand function $G$ are typically differentiable. Non-differentiabilities occur at all prices $q$ for which at least one agent is on the edge between supplying and not supplying an asset, or on the edge between buying and not buying an asset. Because of positive transaction costs there are nondegenerate regions of prices where some agents are not active in at least one of the asset markets. Even with only small transaction costs, this phenomenon may occur at equilibrium prices thereby leading to robust degeneracies because an agent who is not active in a certain asset market is typically not affected by changes in the price of that asset. Robust continua of equilibria may be expected when all agents stop trading in a certain asset. Both the non-differentiabilities and the degeneracies cause difficulties in our proofs and in the numerical implementation of our algorithm.

\section{The Homotopy Approach}

In this section we give a general description of our algorithmic approach. In particular we motivate our approach by pointing out the consequences of the depicted difficulties for the computation of equilibria. We show how to circumvent the detected problems and summarize the main results in a number of propositions which will follow from the results in the next section. We focus on the intuition behind these results and finish this section with a conceptual description of our algorithm.

Our algorithm is based on the homotopy principle which has been successfully applied to the computation of equilibria in general equilibrium models with incomplete markets, see Brown, DeMarzo, and Eaves (1996) and Schmedders (1998, 1999). For an introduction to homotopy methods see Garcia and Zangwill (1981) and Eaves and Schmedders (1999). Here we give just a concise review of the general approach of the homotopy principle as it relates to our problem.

Following the general homotopy approach a natural homotopy for computing equilibria in our model would be the function $F:[0,1] \times Q \rightarrow \mathbb{R}^{J}$ which is defined by

$$
F(t, q)=t G(q)+(1-t)\left(q^{0}-q\right), \quad(t, q) \in[0,1] \times Q,
$$

where $q^{0}$ may be any price system in $Q$. Note that for $t=0$, there is a unique solution, $q=q^{0}$. For $t=1$, the problem $F(1, q)=0$ is equal to the problem $G(q)=0$, and therefore solving $F(1, q)=0$ amounts to finding an equilibrium asset price vector $q$. The idea now would be to start at the unique solution for $F(0, q)=0$ and to follow a path of solutions to $F(t, q)=0$ until eventually a solution to $F(1, q)=0$ is reached.

\footnotetext{
${ }^{3}$ Using standard methods one can now proceed to prove the existence of a competitive equilibrium. Extending the work of Hens (1991) to our model with transaction costs one can show that the asset demand functions $g^{h}$ are continuous on the set $Q$ and that they satisfy a properness condition, see Proposition 3.5. Proposition 3.6 shows that these properties are inherited by the total asset demand function $G$, which is sufficient to show the existence of an equilibrium.
} 
When $F$ is a twice continuously differentiable function the following conditions are sufficient for the convergence of the algorithm (see Garcia and Zangwill (1981)).

(i) $F^{-1}(\{0\}) \cap(\{0\} \times Q)$ has a unique solution.

(ii) $F(0, \cdot) 历\{0\}$ and $F(1, \cdot) 历\{0\}$.

(iii) $F 历\{0\}$.

(iv) $F^{-1}(\{0\})$ is compact.

Under (i)-(iv) it follows that $F^{-1}(\{0\})$ is a compact 1-dimensional differentiable manifold with boundary, so $F^{-1}(\{0\})$ is a finite collection of disjoint paths and loops. ${ }^{4}$ Moreover, the boundary of $F^{-1}(\{0\})$ equals the intersection of $F^{-1}(\{0\})$ and the boundary of $[0,1] \times Q$, so it consists of all $(t, q)$ such that $t=0$ or $t=1$, and $F(t, q)=0$. Since $F^{-1}(\{0\}) \cap(\{0\} \times Q)$ has a unique solution, that solution must be an end-point of a path with another end-point. The only possibility is that the other end-point belongs to $F^{-1}(\{0\}) \cap(\{1\} \times Q)$, so it is a competitive equilibrium price system. A minor problem in the argument is that conditions (ii) and (iii) are usually not satisfied for all economies. The standard approach (Debreu (1970)) is to invoke methods from transversality theory such as Sard's theorem to show that these conditions are satisfied for a generic set of economies.

When we try to apply the standard homotopy approach to finding equilibria in our model we encounter two significant problems. The first difficulty is the existence of non-differentiabilities of the homotopy $F$, which are due to non-differentiabilities in the market excess demand function $G$. We approach this problem by subdividing the domain of $F$ into subsets where the excess demand $G$ and thus the homotopy $F$ are differentiable. The zero sets of $F$ on the different subsets are then nicely tied together to guarantee the convergence of the algorithm. The second difficulty is that even the application of transversality theory cannot rule out the occurrence of robust degeneracies which occur at $t=1$. Notice that (i)-(iv) imply the existence of a finite number of equilibrium price systems. With transaction costs it cannot be ruled out that certain assets are robustly non-traded by every trader, even at equilibrium prices. In that case there is a continuum of equilibrium prices, as small perturbations in prices of assets that nobody trades in, do not affect market clearing. The solutions to the homotopy equations cannot even be expected to constitute a 1-dimensional topological manifold. For example, if two or more assets are robustly non-traded, then the prices of all these assets can vary within some neighborhood without affecting the equilibria.

\subsection{The Int uitive Homotopy}

We solve the robust degeneracy problem by making an equilibrium selection. The idea of equilibrium selection in a competitive framework has been exploited in Dubey, Geanakoplos and Shubik (1997) but it is rarely used in a competitive equilibrium analysis. We analyze two alternatives for

\footnotetext{
${ }^{4} \mathrm{~A}$ path is a set diffeomorphic to the unit interval and a loop is a set diffeomorphic to the unit circle.
} 
equilibrium selection. The first alternative considers only those competitive equilibria where for each asset market there is non-zero trade, or at least one agent is indifferent between selling an asset and not selling an asset. The second alternative considers those competitive equilibria where for each asset market there is non-zero trade, or at least one agent is indifferent between buying and not buying an asset.

We say that an agent $h$ is indifferent at $q$ between selling asset $j$ and being inactive in asset market $j$ if $g_{j}^{h}(q)=0$ and the relaxation of the non-negativity constraint on $\theta_{j}^{h,-}$ would not affect the optimal decision of household $h$. Under the differentiability assumptions made the latter condition is equivalent to the requirement that the Lagrange multiplier corresponding to the inequality $\theta_{j}^{h,-} \geq 0$ equals zero at $q$. This Lagrange multiplier is denoted by $\lambda_{j}^{h,-}(q)$ and equals $-\partial_{c_{0}^{h}} u^{h}\left(c^{h}(q)\right)\left(q_{j}-k_{j}\right)+$ $\sum_{s=1}^{S} \partial_{c_{s}^{h}} u^{h}\left(c^{h}(q)\right) d_{s}^{j}$. A similar definition applies for an agent to be indifferent between buying asset $j$ and being inactive in asset market $j$. The Lagrange multiplier corresponding to the inequality $\theta_{j}^{h,+} \geq 0$ is denoted by $\lambda_{j}^{h,+}(q)$ and equals $\partial_{c_{0}^{h}} u^{h}\left(c^{h}(q)\right)\left(q_{j}+k_{j}\right)-\sum_{s=1}^{S} \partial_{c_{s}^{h}} u^{h}\left(c^{h}(q)\right) d_{s}^{j}$. The set of agents which is indifferent between selling asset $j$ and not selling asset $j$ is denoted by $I_{j}^{-}(q)$ and the set of agents which is indifferent between buying asset $j$ and not buying asset $j$ by $I_{j}^{+}(q)$. The demand function of agents in $I_{j}^{-}(q) \cup I_{j}^{+}(q)$ displays a non-differentiability at the asset price system $q$. It is due to this non-differentiability that robust non-degeneracies may occur.

Definition 3.1: A competitive equilibrium $\left(\theta^{*}, q^{*}\right)$ of $\mathcal{E}$ is demand-perfect, if in each asset market $j$ there is non-zero trade, $\theta_{j}^{* h} \neq 0$ for some $h$, or at least one agent is indifferent between selling asset $j$ and not selling asset $j, I_{j}^{-}\left(q^{*}\right) \neq \emptyset$.

The terminology demand-perfect comes from the fact that generically demand-perfect equilibria are the ones that are robust to the introduction of a trader that demands all assets. Indeed, generically, demand-perfect equilibria are obtained by perturbing the total excess demand function of the economy by an excess demand function of a trader that demands all assets, considering the set of competitive equilibria that result, and taking the limit of the set of competitive equilibria for a perturbation going to zero. For each asset market, it holds in the limit either that there is non-zero trade or that one agent will be on the limit of supplying the asset or not supplying the asset. Indeed, in the perturbed economy this household supplies a tiny amount to clear the market, and in the limit it is indifferent between supplying and not supplying. A similar motivation can be given for supply-perfect equilibria. From an economic point of view, no information is lost by restricting attention to supply-perfect or demand-perfect equilibria, in the sense that no competitive equilibrium allocations are lost.

Proposition 3.2: For each competitive equilibrium $\left(\theta^{*}, q^{*}\right)$ of $\mathcal{E}$ there is exactly one allocationally equivalent supply-perfect equilibrium $\left(\theta^{*}, q^{\mathrm{s}}\right)$ and exactly one allocationally equivalent demand-perfect equilibrium $\left(\theta^{*}, q^{\mathrm{d}}\right)$.

The proof of Proposition 3.2 yields another important aspect of supply-perfect and demand- 
perfect equilibria. These equilibria give a lower bound, respectively an upper bound, on prices that sustain a certain allocation. We exploit this property in our numerical examples, to give the equilibrium interval of asset prices in case there is no trade in a certain asset.

We modify the natural homotopy to compute demand-perfect equilibria. To this end we define, for $q \in Q$,

$$
\Lambda_{j}^{-}(q)=\prod_{h=1}^{H} \lambda_{j}^{h,-}(q), \quad j=1, \ldots, J .
$$

We now add the term $\frac{\varphi\left(\Lambda_{j}^{-}(q)\right)}{1+e^{q_{j}}}$ to the total excess demand function $G_{j}$, where $\varphi: \mathbb{R} \rightarrow \mathbb{R}$ is any bounded, differentiable function with $\varphi(0)=0$ and $\varphi^{\prime}>0$. The function $1+e^{q_{j}}$ in the denominator is used for simplicity of the proofs. Any function of $q_{j}$ with an everywhere nonnegative derivative that diverges to plus infinity as $q_{j}$ tends to plus infinity would suffice. This addition results in the function $\widetilde{G}$, defined by

$$
\widetilde{G}_{j}(q)=G_{j}(q)+\frac{\varphi\left(\Lambda_{j}^{-}(q)\right)}{1+e^{q_{j}}}, \quad j \in J, q \in Q
$$

Proposition 3.3: It holds that $\left(g^{1}\left(q^{\mathrm{d}}\right), \ldots, g^{H}\left(q^{\mathrm{d}}\right), q^{\mathrm{d}}\right)$ is a demand-perfect competitive equilibrium of $\mathcal{E}$ if and only if $\widetilde{G}\left(q^{\mathrm{d}}\right)=0$.

The intuitive homotopy is the function $\widetilde{F}:[0,1] \times Q \rightarrow \mathbb{R}^{J}$ defined by

$$
\widetilde{F}(t, q)=t \widetilde{G}(q)+(1-t)\left(q^{0}-q\right), \quad(t, q) \in[0,1] \times Q,
$$

where $q^{0}$ may be any price system in $Q$. Note that for $t=0$, there is a unique solution, $q=q^{0}$. For $t=1$, the problem $\widetilde{F}(1, q)=0$ is equal to the problem $\widetilde{G}(q)=0$, and therefore, by Proposition 3.3 , solving $\widetilde{F}(1, q)=0$ amounts to finding an asset price vector $q$ that induces a demand-perfect equilibrium. The idea now would be to start at the unique solution for $\widetilde{F}(0, q)=0$ and to follow a path of solutions to $\widetilde{F}(t, q)=0$ until eventually a solution to $\widetilde{F}(1, q)=0$ is reached.

\subsection{Bid-Ask Structures}

The remaining problem is the existence of non-differentiabilities of $\widetilde{F}$. The basic idea is to subdivide the domain of $\widetilde{F}$ into subsets on which $\widetilde{F}$ is differentiable, and to jump from one such subset to the next when tracking the solution curve of $\widetilde{F}$ from $\left(0, q^{0}\right)$ to a demand-perfect equilibrium. This approach is supported by the fact that generically these subsets are a covering of $Q$, that is, the relative interiors of any two such subsets have an empty intersection, and the union of all those subsets equals the set $Q$. The standard homotopy approach allows us to follow a path of solutions in any of these subsets. Once we hit a boundary of our domain subset we switch over to the next subset and continue the path. In order to formalize this idea we need some further notation. 
We define a set $R$ of sign vectors,

$$
R=\left\{r \in \mathbb{R}^{H J} \mid r_{j}^{h} \in\{-1,0,+1\}\right\} .
$$

A sign vector $r \in R$ determines a subset of $Q$ where the sign of the trades being made, the bid-ask structure, is determined by $r$. If $r_{j}^{h}=-1$, then agent $h$ supplies asset $j$, if $r_{j}^{h}=0$ then agent $h$ does not trade in asset market $j$, and if $r_{j}^{h}=+1$, then agent $h$ is buying asset $j$.

Formally, for $r \in R$,

$$
\begin{aligned}
Q(r)=\{q \in Q \mid & g_{j}^{h}(q)<0, \text { or } g_{j}^{h}(q)=0 \text { and } h \in I_{j}^{-}(q), \text { when } r_{j}^{h}=-1, \\
& g_{j}^{h}(q)=0, \text { when } r_{j}^{h}=0, \\
& \left.g_{j}^{h}(q)>0, \text { or } g_{j}^{h}(q)=0 \text { and } h \in I_{j}^{+}(q), \text { when } r_{j}^{h}=+1\right\},
\end{aligned}
$$

and $g^{h, r}: Q(r) \rightarrow \mathbb{R}^{J}$ and $G^{r}: Q(r) \rightarrow \mathbb{R}^{J}$ denote the restrictions of the individual demand functions for assets and the total demand function for assets to $Q(r)$. For all $q \in Q(r)$ an agent $h$ is always taking a long position in the same set of assets, a short position in the same set of assets, and not trading at all in the same set of assets. The fact that the long or short position could go to zero complicates the definition somewhat. Furthermore, $\Lambda^{-r}: Q(r) \rightarrow \mathbb{R}^{J}$ denotes the restriction of $\Lambda^{-}$to $Q(r)$. Notice that $\Lambda_{j}^{-r}$ is identically equal to zero if $r_{j}^{h}=-1$ for at least one household $h$.

Proposition 3.4: For $r \in R$, the asset demand functions $g^{h, r}$ and $G^{r}$, and the function $\Lambda^{-r}$ are twice continuously differentiable. ${ }^{5}$

The next observation concerns the asset demand functions. If an asset price ever approaches an arbitrage price, then the asset demand will diverge. This property will prove crucial in the convergence proof of our algorithm. We denote the excess demand for the consumption good by agent $h$ at date 0 by $g_{0}^{h}$, so $g_{0}^{h}(q)=c_{0}^{h}-e_{0}^{h}$ with $c^{h}$ the utility maximizing consumption bundle at prices $q$. We define $Q^{0}$ as the set of no-arbitrage asset prices in the absence of transaction costs, so $Q^{0} \subset Q$.

Proposition 3.5: Let $q^{n} \in Q$ be any sequence converging to $\bar{q} \in \partial Q$ or diverging, that is, $\left\|q^{n}\right\| \rightarrow \infty$. Then $\left\|\left(g_{0}^{h}\left(q^{n}\right), g^{h}\left(q^{n}\right)\right)\right\| \rightarrow \infty$. Moreover, for any $q^{0} \in Q^{0},\left(1, q^{0}\right) \cdot\left(g_{0}^{h}\left(q^{n}\right), g^{h}\left(q^{n}\right)\right) \rightarrow$ $+\infty$.

The latter part of Proposition 3.5 will prove most important, and carries over to aggregate asset demand functions.

Proposition 3.6: Let $q^{n} \in Q$ be any sequence converging to $\bar{q} \in \partial Q$ or diverging, that is, $\left\|q^{n}\right\| \rightarrow \infty$. Then, for any $q^{0} \in Q^{0},\left(1, q^{0}\right) \cdot\left(G_{0}\left(q^{n}\right), G\left(q^{n}\right)\right) \rightarrow+\infty$.

\footnotetext{
${ }^{5}$ A function with domain a subset of Euclidean space which is not necessarily open is differentiable if it has a differentiable extension to an open neighborhood of its domain of definition.
} 
Next we define the set $P(r)$ consisting of the pairs of the homotopy parameter and the asset price vectors that satisfy the homotopy equation, together with the requirement that $r$ be compatible with the bid-ask structure in all markets, that is,

$$
P(r)=\{(t, q) \in[0,1] \times Q(r) \mid \widetilde{F}(t, q)=0\} .
$$

Recall our definition of the open set $E=\mathbb{R}_{++}^{(1+S) H}$. We now have the following result.

THEOREM 3.7: There is a subset $E^{*}$ of $E$ such that $E \backslash E^{*}$ has a closure with Lebesgue measure zero and for all $e \in E^{*}$, for all $r \in R, P(r)$ is a compact, 1-dimensional $C^{2}$ manifold with boundary. A point $(t, q)$ in the boundary of $P(r)$ is either not a boundary point of $P(\bar{r})$ for all $\bar{r} \neq r$ and belongs to $\{0,1\} \times Q$, or is a boundary point of exactly one $P(\bar{r})$ with $\bar{r} \neq r$ and belongs to $(0,1) \times Q$. Moreover, $r$ and $\bar{r}$ differ in exactly one element which changes from -1 to 0 or from +1 to 0 , or the reverse.

For almost all economies, for all $r \in R$, the set $P(r)$ is a compact, 1-dimensional differentiable manifold with boundary, so it is a finite collection of disjoint paths and loops. It follows that each component of $P(r)$, i.e. a maximally connected subset of $P(r)$, is either a path or a loop. We write $P(r)=P(r, 1) \cup \cdots \cup P(r, c(r))$, where $P(r, c), c=1, \ldots, c(r)$, is a component of $P(r)$ and $c(r)$ is the number of components in $P(r)$. The set $P=\cup_{r \in R} P(r)$ is the set of all potential solutions to the homotopy equations, so $P=\widetilde{F}^{-1}(\{0\})$.

As a corollary to the results in the Appendix we get the following result, which confirms that the non-differentiabilities of $\widetilde{F}$ are well-behaved and do lead to well-behaved non-differentiabilities of $\widetilde{F}^{-1}(\{0\})$ that allow us to prove convergence of our algorithm.

THEOREM 3.8: There is a subset $E^{*}$ of $E$ such that $E \backslash E^{*}$ has a closure with Lebesgue measure zero and such that for all $e \in E^{*}$ the following statements hold. The set $\widetilde{F}^{-1}(\{0\})$ is a compact 1-dimensional piecewise $C^{2}$ manifold with boundary. ${ }^{6}$ The boundary of $\widetilde{F}^{-1}(\{0\})$ equals the intersection of $F^{-1}(\{0\})$ and $\{0,1\} \times Q$ and is a compact 0 -dimensional manifold. There is a unique boundary point in $\{0\} \times Q$.

The next subsection explains on an intuitive level how Theorem 3.8 is obtained.

\subsection{The Essence of the Method}

The unique starting point is given by $q=q^{0}$, and corresponds to a bid-ask structure $r^{0}$ that is given by $r^{0}=\operatorname{sign}\left(g\left(q^{0}\right)\right)$. It holds that $\left(0, q^{0}\right) \in P\left(r^{0}, c^{0}\right)$ for some $c^{0} \in\left\{1, \ldots, c\left(r^{0}\right)\right\}$. The set $P\left(r^{0}, c^{0}\right)$ is either a path or a loop. Since $\left(0, q^{0}\right)$ is a boundary point of $P\left(r^{0}, c^{0}\right)$, it is a path and must possess another end point, say $\left(t^{1}, q^{1}\right)$. If $t^{1}=1$, then a demand-perfect competitive equilibrium has been found. If $t^{1}<1$, then the reason that we cannot continue following the path $P\left(r^{0}, c^{0}\right)$ is that we are at a boundary point of $Q\left(r^{0}\right)$.

\footnotetext{
${ }^{6} \mathrm{~A}$ manifold is a 1-dimensional piecewise $C^{2}$ manifold if it is a 1-dimensional topological manifold that is a finite union of $C^{2}$ manifolds.
} 
Either exactly one agent $h^{\prime}$ for which $r_{j^{\prime}}^{0 h^{\prime}}=0$ belongs to $I_{j^{\prime}}^{-}\left(q^{1}\right)$ or $I_{j^{\prime}}^{+}\left(q^{1}\right)$, or there is exactly one agent $h^{\prime}$ for which $r_{j^{\prime}}^{0 h^{\prime}}=-1$ or $r_{j^{\prime}}^{0 h^{\prime}}=+1$, such that $g_{j^{\prime}}^{h^{\prime}}\left(q^{1}\right)=0$. In the first case an inactive agent is on the edge of becoming an active trader in the market for asset $j^{\prime}$. In the second case an active agent becomes inactive in the market for asset $j^{\prime}$. In both cases it holds that $q^{1} \in Q\left(r^{1}\right)$, where, in the first case, $r^{1}$ is such that $r_{j^{\prime}}^{1 h^{\prime}}=0$ and $r_{j}^{1 h}=r_{j}^{0 h},(h, j) \neq\left(h^{\prime}, j^{\prime}\right)$, and, in the second case, $r^{1}$ is such that $r_{j^{\prime}}^{1 h^{\prime}}=-1$ or $r_{j^{\prime}}^{1 h^{\prime}}=+1$, and $r_{j}^{1 h}=r_{j}^{0 h},(h, j) \neq\left(h^{\prime}, j^{\prime}\right)$.

The key insight is that $\left(t^{1}, q^{1}\right) \in P\left(r^{1}, c^{1}\right)$ for exactly one $c^{1} \in\left\{1, \ldots, c\left(r^{1}\right)\right\}$, and is a boundary point of $P\left(r^{1}, c^{1}\right)$. Therefore, $P\left(r^{1}, c^{1}\right)$ is a path and should have another end point, say $\left(t^{2}, q^{2}\right)$. Now, again, either $t^{2}=1$ and a demand-perfect equilibrium has been found, or $t^{2}<1$ and it holds that $q^{2}$ is a boundary point of a uniquely determined $P\left(r^{2}, c^{2}\right)$.

The general argument is as follows. The homotopy will generate a sequence of components $\left\{P\left(r^{n}, c^{n}\right)\right\}_{n=1, \ldots, N}$, where $N$ might (still) be infinite. The sequence $\left(r^{n}\right)_{n=1, \ldots, N}$ is alternating; it cannot happen that two components $P(r, c)$ and $P(r, \bar{c})$ of the same manifold $P(r)$ are generated consecutively. Moreover, from a certain bid-ask structure $r \in R$, the next bid-ask structure $\bar{r} \in R$ found by the homotopy differs from $r$ in exactly one component. Either some $r_{j}^{h}=0$ and $\bar{r}_{j}^{h}$ is -1 or +1 , or some $r_{j}^{h}$ is -1 or +1 and $\bar{r}_{j}^{h}=0$. In economic terms, either an agent $h$ that was inactive in asset market $j$, starts supplying or demanding asset $j$, or an agent $h$ that was short or long in asset market $j$, gets inactive.

The total number of different components $P(r, c)$ is finite, as the number of bid-ask structures is finite and, by Theorem 3.7, each bid-ask structure leads to a finite number of components. Hence, the sequence $\left\{P\left(r^{n}, c^{n}\right)\right\}_{n=1, \ldots, N}$ is a finite sequence, or one component $P(\bar{r}, \bar{c})$ is the first to be generated for the second time. In the first case, the last end point found, say $\left(t^{N}, q^{N}\right)$ satisfies that $t^{N}=1$ and $q^{N}$ is a demand-perfect equilibrium. (If $t^{N}<1$, it should have been the starting point of another component.) The second case cannot occur, as it would be inconsistent with the door-in door-out principle of Lemke and Howson (1964). In order for a component $P(\bar{r}, \bar{c})$ to be regenerated by the algorithm, it has to be connected to at least two other components, which is shown to be impossible in the Appendix. This argument shows both generic convergence of the algorithm and that $P$ has a component which is a path, connecting $q^{0}$ to a demand-perfect equilibrium.

The set $P$ is a compact, 1-dimensional piecewise $C^{2}$ manifold with boundary, so it consists of a finite number of paths and loops. Loops contain no competitive equilibria. There may be no loops at all. Paths come in two sorts. There is a unique path starting at $\left(0, q^{0}\right)$ and terminating at $\left(1, q^{* 1}\right)$, where $q^{* 1}$ is a demand-perfect competitive equilibrium. This path is piecewise $C^{2}$, our algorithm is convergent. All other paths have two end points at $t=1$, so connect two different demand-perfect competitive equilibria. We get the following corollary.

Corollary 3.9: There is a subset $E^{*}$ of $E$ such that $E \backslash E^{*}$ has a closure with Lebesgue measure zero and for all $e \in E^{*}, P$ consists of one path connecting $\left(0, q^{0}\right)$ and $\left(1, q^{* 1}\right)$ where $q^{* 1}$ is a demand-perfect equilibrium, so the homotopy algorithm is convergent, consists of a finite number of paths connecting two different demand-perfect equilibria, and consists of a finite number of loops without equilibria. The number of demand-perfect equilibria is odd. 
The homotopy we have used so far is well suited for the intuitive discussion of the technical difficulties in this model. Many general equilibrium theorists like to use excess demand functions for their arguments. Therefore, a homotopy using excess demand functions appears to be a natural choice for the discussion of the approach. However, both for proving convergence of our algorithm as well as for an implementation, a homotopy with excess demand functions proves to be impractical. When asset markets are incomplete, excess demand functions are notoriously hard to compute. In our case this problem is compounded by the presence of transaction costs. These problems lead us to two different homotopies which we discuss in the next two sections. In particular, we treat two complications. The first is the difficulty of getting closed-form solutions for demand functions. The second is the potentially huge number of switches between several pieces $P(r, c)$.

\section{The Theoretical Homotopy}

The very serious difficulty of finding a closed form solution for the total demand function typically receives very little attention. Nevertheless, we are not aware of any utility function satisfying the standard monotonicity and concavity assumptions for which closed-form solutions are available in the presence of both transaction costs and incomplete markets. In fact, even in the case without transaction costs, Brown, DeMarzo and Eaves (1996a) had to resort to a not everywhere monotonic utility function, in order to get a closed-form expression. However, the homotopy approach makes it possible to tackle this problem in an elegant way. Instead of defining the homotopy by means of the total demand function for assets $G$, the homotopy is defined in terms of the first-order equations that characterize demand, see Garcia and Zangwill (1981) and Schmedders (1998). We need some additional notation to give such a formulation.

Given a sign vector $r \in R$ we are interested in the collection of assets for which agent $h$ is a supplier, the assets in which he does not trade, and the assets for which agent $h$ acts as a buyer. These sets are denoted by $J_{h}^{-}(r), J_{h}^{0}(r)$, and $J_{h}^{+}(r)$, so

$$
\begin{aligned}
J_{h}^{-}(r) & =\left\{j \in J \mid r_{j}^{h}=-1\right\}, \\
J_{h}^{0}(r) & =\left\{j \in J \mid r_{j}^{h}=0\right\}, \\
J_{h}^{+}(r) & =\left\{j \in J \mid r_{j}^{h}=+1\right\} .
\end{aligned}
$$

The following notation indicates for each sign vector $r \in R$ all combinations of agents and assets where supply, inactivity or demand occurs,

$$
\begin{aligned}
R^{-}(r) & =\left\{(h, j) \in H \times J \mid r_{j}^{h}=-1\right\}, \\
R^{0}(r) & =\left\{(h, j) \in H \times J \mid r_{j}^{h}=0\right\}, \\
R^{+}(r) & =\left\{(h, j) \in H \times J \mid r_{j}^{h}=+1\right\} .
\end{aligned}
$$

Consider any sign vector $r \in R$ and any $(t, q) \in \mathbb{R} \times Q$, then $(t, q) \in P(r)$ if and only if there is 


$$
\begin{aligned}
\left(\lambda^{-}, \lambda^{+}, \theta, c\right) \in \mathbb{R}^{H J} \times \mathbb{R}^{H J} \times \mathbb{R}^{H J} \times \mathbb{R}^{H(1+S)} \text { such that } & \\
\lambda_{j}^{h,-}=0, & (h, j) \in R^{-}(r), \\
\lambda_{j}^{h,+}=0, & (h, j) \in R^{+}(r), \\
\theta_{j}^{h}=0, & (h, j) \in R^{0}(r), \\
c_{0}^{h}-e_{0}^{h}+\sum_{j \in J_{h}^{-}(r)} \theta_{j}^{h}\left(q_{j}-k_{j}\right)+\sum_{j \in J_{h}^{+}(r)} \theta_{j}^{h}\left(q_{j}+k_{j}\right)=0, \quad h \in H, & \\
c_{s}^{h}-e_{s}^{h}-\sum_{j \in J_{h}^{-}(r) \cup J_{h}^{+}(r)} \theta_{j}^{h} d_{s}^{j}=0, & h \in H, s \in S, \\
\lambda_{j}^{h,-}+\partial_{c_{0}^{h}} u^{h}\left(c^{h}\right)\left(q_{j}-k_{j}\right)-\sum_{s=1}^{S} \partial_{c_{s}^{h}} u^{h}\left(c^{h}\right) d_{s}^{j}=0, & h \in H, j \in J, \\
\lambda_{j}^{h,+}-\partial_{c_{0}^{h}} u^{h}\left(c^{h}\right)\left(q_{j}+k_{j}\right)+\sum_{s=1}^{S} \partial_{c_{s}^{h}} u^{h}\left(c^{h}\right) d_{s}^{j}=0, & h \in H, j \in J, \\
\varphi\left(\prod_{h=1}^{H} \lambda_{j}^{h,-}\right) & \\
1+e^{q_{j}}=0, & j \in J, \\
\lambda_{j}^{h,-} \geq 0, & (h, j) \in R^{0}(r), \\
\lambda_{j}^{h,+} \geq 0, & (h, j) \in R^{0}(r), \\
-\theta_{j}^{h} \geq 0, & (h, j) \in R^{-}(r), \\
\theta_{j}^{h} \geq 0, & (h, j) \in R^{+}(r), \\
\theta_{j}^{h}+(1-t)\left(q_{j}^{0}-q_{j}\right)+t \frac{1}{t} \geq 0, & \\
1-t \geq 0 . &
\end{aligned}
$$

Equations (8) are the perturbed market-clearing conditions that correspond to the intuitive homotopy equations $\widetilde{F}(t, q)=0$, only that the asset-demand functions are replaced by the portfolio choices $\theta_{j}^{h}$. Note that equations (8) are the only equations containing the homotopy parameter $t$ that is constrained to lie between 0 and 1 by inequalities (13)-(14). Equations (1)-(7) and inequalities (9)-(12) are the first-order conditions of the agents' utility maximization problems. These conditions are necessary and sufficient since the agents' utility maximization problems are convex programming problems with linear constraints, so a constraint qualification is satisfied. In fact, the first-order conditions of the agents' utility maximization problems also lead to the inequalities $\lambda_{j}^{h,^{-}} \geq 0$ for $(h, j) \in R^{+}(r)$ and $\lambda_{j}^{h,+} \geq 0$ for $(h, j) \in R^{-}(r)$. These inequalities are redundant, as they follow with strict inequality from equations (1), (6) and (7), and (2), (6) and (7), respectively, making use of the assumption that $\partial_{c_{0}^{h}} u^{h}\left(c^{h}\right)$ and $k_{j}$ are strictly positive. These inequalities are therefore omitted. Equations (4) and (5) are the budget constraints, equations (6) and (7) are the derivatives with respect to the decision variables $\theta_{j}^{h}$, and equations (1)-(3) are the complementary slackness conditions for the multipliers corresponding to the sign constraints on the decision variables. Note that for $(h, j) \in R^{0}(r)$ the complementarity condition reduces simply to $\theta_{j}^{h}=0$, that is, to equation (3). If $\theta_{j}^{h}<0$, then $\lambda_{j}^{h,-}$ must be 0 , and the complementarity condition is just equation (1). Inequalities (9)-(12) are the sign restriction on the decision variables and multipliers. 
The number of variables $\left(t, q, \lambda^{-}, \lambda^{+}, \theta, c\right)$ in the system of equations (1)-(8) equals $1+J+2 H J+$ $H J+H(1+S)$, one more than the number of equations which is given by $H J+H(1+S)+2 H J+J$. Typically, we would expect a one-dimensional set of solutions. If, in addition to (1)-(8), we require exactly one of the inequalities in (9)-(14) to hold with equality, we expect to obtain generically a finite set of locally unique solutions. This intuition is confirmed by formal proofs in the Appendix. For $r \in R$, the solutions to the system of equations (1)-(8) and the inequalities (9)-(14) is denoted by $\widetilde{P}(r)$. In the Appendix it is shown that $\widetilde{P}(r)$ is a compact, differentiable 1-dimensional manifold with boundary. It is in this way that we show that $P(r)$ is a 1-dimensional manifold with boundary as the following proposition states that the two sets are diffeomorphic.

Proposition 4.1: For $r \in R, P(r)$ and $\widetilde{P}(r)$ are $C^{2}$ diffeomorphic.

THEOREM 4.2: There is a subset $E^{*}$ of $E$ such that $E \backslash E^{*}$ has a closure with Lebesgue measure zero and for all $e \in E^{*}$, for all $r \in R, \widetilde{P}(r)$ is a compact, 1-dimensional $C^{2}$ manifold with boundary. $A$ point $\left(t, q, \lambda^{-}, \lambda^{+}, \theta, c\right)$ in the boundary of $\widetilde{P}(r)$ is either not a boundary point of $\widetilde{P}(\bar{r})$ for all $\bar{r} \neq r$ and belongs to $\{0,1\} \times Q \times \mathbb{R}^{H J} \times \mathbb{R}^{H J} \times \mathbb{R}^{H J} \times \mathbb{R}^{H(1+S)}$, or is a boundary point of exactly one $\widetilde{P}(\bar{r})$ with $\bar{r} \neq r$ and belongs to $(0,1) \times Q \times \mathbb{R}^{H J} \times \mathbb{R}^{H J} \times \mathbb{R}^{H J} \times \mathbb{R}^{H(1+S)}$. Moreover, $r$ and $\bar{r}$ differ in exactly one element which changes from -1 to 0 or from +1 to 0 , or the reverse.

Theorem 3.7 is a corollary to Proposition 4.1 and Theorem 4.2. Once we have shown that $\widetilde{P}(r)$ is a $C^{2}$ manifold with boundary, and that there is a $C^{2}$ diffeomorphism between $\widetilde{P}(r)$ and $P(r)$, it follows that $P(r)$ is a manifold with boundary, where the diffeomorphism maps the boundary of $\widetilde{P}(r)$ to the boundary of $P(r)$, see Jongen, Jonker and Twilt (1983), Corollary 3.1.3, page 103. The way to generate the homotopy path is similar to before. The major difference is that the, usually not available, closed form expression for $\widetilde{G}$ needed for the homotopy $\widetilde{F}$, is not needed anymore. The homotopy $\widetilde{F}$ is replaced by the equations $(1)-(8)$.

\subsection{Observations}

Before we continue the discussion of the development of our three homotopies we make a few observations about the homotopy-path following using the theoretical homotopy.

Multiplying equations (5) with some vector of state prices $\pi \in \mathbb{R}_{++}^{S}$ satisfying the condition of Proposition 2.2 and adding equation (4) results in the inequality

$$
\left(c_{0}^{h}-e_{0}^{h}\right)+\sum_{s=1}^{S} \pi_{s}\left(c_{s}^{h}-e_{s}^{h}\right) \leq 0 \quad \text { for all } h \in H,
$$

with equality if only if the agent doesn't trade on the financial markets or in the special case $k=0$. In both cases the agent doesn't "burn" any resources on the financial markets because he doesn't incur transaction costs. The latter case just reflects the Cass trick for GEI economies without transaction costs. Note, that the above inequality holds along the entire homotopy path and not only in equilibrium. 
Adding equations (6) and (7) yields the following equation

$$
\lambda_{j}^{h,-}+\lambda_{j}^{h,+}=2 \partial_{c_{0}^{h}} u^{h}\left(c^{h}\right) k_{j} .
$$

This equation shows that $\lambda_{j}^{h,-}+\lambda_{j}^{h,+}>0$ implying the last statement of Proposition 2.3, namely that $\theta_{j}^{h,-} \cdot \theta_{j}^{h,+}=0$, it can never be optimal for an agent to be both long and short in a financial security. Moreover, this equation sheds light on what happens along the homotopy path when an agent changes sides on a security market, for example, from being long, to being inactive, to being short. When the agent's long position in asset $j$ is reduced down to zero, and the homotopy path is at the boundary of two sets $P(r)$ and $P\left(r^{\prime}\right)$ the shadow prices are $\lambda_{j}^{h,-}=2 \partial_{c_{0}^{h}} u^{h}\left(c^{h}\right) k_{j}$ and $\lambda_{j}^{h,+}=0$. As the homotopy path moves through the interior of $P\left(r^{\prime}\right)$ both shadow prices are positive indicating by complementary slackness that the asset variable is zero, that is $\theta_{j}^{h}=0$, or equivalently $\theta_{j}^{h,-}=\theta_{j}^{h,+}=0$. As the path hits a set $P\left(r^{\prime \prime}\right)$ where the agent is short in asset $j$ (in its interior) the shadow prices reach the point where $\lambda_{j}^{h,-}=0$ and $\lambda_{j}^{h,+}=2 \partial_{c_{0}^{h}} u^{h}\left(c^{h}\right) k_{j}$.

In addition, we can infer some properties of demand- and supply-perfect equilibria from equations (6) and (7). The shadow prices always satisfy $\lambda_{j}^{h,-}, \lambda_{j}^{h,+} \geq 0$ resulting in the following inequalities for all $h \in H$,

$$
q_{j} \leq k_{j}+\sum_{s=1}^{S}\left(\frac{\partial_{c_{s}^{h}} u^{h}\left(c^{h}\right)}{\partial_{c_{0}^{h}} u^{h}\left(c^{h}\right)} d_{s}^{j}\right), \quad \text { and } \quad q_{j} \geq-k_{j}+\sum_{s=1}^{S}\left(\frac{\partial_{c_{s}^{h}} u^{h}\left(c^{h}\right)}{\partial_{c_{0}^{h}} u^{h}\left(c^{h}\right)} d_{s}^{j}\right) .
$$

Hence, the price range for asset $j$ in equilibrium equals

$$
-k_{j}+\max _{h \in H}\left\{\sum_{s=1}^{S} \frac{\partial_{c_{s}^{h}} u^{h}\left(c^{h}\right)}{\partial_{c_{0}^{h}} u^{h}\left(c^{h}\right)} d_{s}^{j}\right\} \leq q_{j} \leq k_{j}+\min _{h \in H}\left\{\sum_{s=1}^{S} \frac{\partial_{c_{s}^{h}} u^{h}\left(c^{h}\right)}{\partial_{c_{0}^{h}} u^{h}\left(c^{h}\right)} d_{s}^{j}\right\} .
$$

An immediate consequence of the last inequalities is that the price difference between the asset prices in a demand-perfect equilibrium and a supply-perfect equilibrium never exceeds $2 k_{j}$. In a demand-perfect equilibrium the upper bound $k_{j}+\min _{h \in H}\left\{\sum_{s=1}^{S} \frac{\partial_{c_{s}^{h}} u^{h}\left(c^{h}\right)}{\partial_{c_{0}^{h}} u^{h}\left(c^{h}\right)} d_{s}^{j}\right\}$ and in a supplyperfect equilibrium the lower bound $-k_{j}+\max _{h \in H}\left\{\sum_{s=1}^{S} \frac{\partial_{c_{s}^{h}} u^{h}\left(c^{h}\right)}{\partial_{c_{0}^{h}} u^{h}\left(c^{h}\right)} d_{s}^{j}\right\}$ is computed.

\section{The Practical Homotopy}

A drawback of our algorithm based on the theoretical homotopy is that a large number of bid-ask structures might be generated before reaching a demand-perfect equilibrium. To avoid this problem we exploit the complementarity between the portfolio variables $\theta_{j}^{h,-}\left(\theta_{j}^{h,+}\right)$ and the shadow prices $\lambda_{j}^{h,-}\left(\lambda_{j}^{h,+}\right)$ of the nonnegativity constraints for the portfolio variables. See also Garcia and Zangwill (1981) and Herings and Peeters (2000) for a discussion of this approach. Equations (1)-(3) and inequalities (9)-(12) imply the standard complementarity conditions

$$
\theta_{j}^{h,-} \cdot \lambda_{j}^{h,-}=0 \text { and } \theta_{j}^{h,+} \cdot \lambda_{j}^{h,+}=0 .
$$


Therefore, we actually can represent, for example, $\theta_{j}^{h,+}$ and $\lambda_{j}^{h,+}$ by a single variable. We introduce two vectors $\alpha^{-}, \alpha^{+} \in \mathbb{R}^{H J}$ and substitute the following functions for the portfolio variables and shadow prices

$$
\begin{aligned}
\lambda_{j}^{h,-} & =\left(\max \left\{0, \alpha_{j}^{h,-}\right\}\right)^{l}, \\
\lambda_{j}^{h,+} & =\left(\max \left\{0, \alpha_{j}^{h,+}\right\}\right)^{l}, \\
\theta_{j}^{h,-} & =\left(\max \left\{0,-\alpha_{j}^{h,-}\right\}\right)^{l}, \\
\theta_{j}^{h,+} & =\left(\max \left\{0,-\alpha_{j}^{h,+}\right\}\right)^{l},
\end{aligned}
$$

where $l$ can be any integer greater than or equal to two. Note that the functions are $l-1$ times continuously differentiable in the variables $\alpha_{j}^{h,-}$ and $\alpha_{j}^{h,+}$, respectively. By definition of these functions inequalities (9)-(12) are always automatically satisfied and we can drop them from consideration.

A bid-ask structure $r$ corresponds to $\left(\alpha^{-}, \alpha^{+}\right) \in A^{-}(r) \times A^{+}(r)$, where

$$
\begin{aligned}
& A^{-}(r)=\left\{\alpha^{-} \in \mathbb{R}^{H J} \mid \begin{array}{l}
\alpha_{j}^{h,-} \leq 0 \text { if }(h, j) \in R^{-}(r) \\
\left.\alpha_{j}^{h,-} \geq 0 \text { if }(h, j) \in R^{0}(r) \cup R^{+}(r)\right\}
\end{array}\right. \\
& A^{+}(r)=\left\{\alpha^{+} \in \mathbb{R}^{H J} \mid \begin{array}{l}
\alpha_{j}^{h,+} \leq 0 \text { if }(h, j) \in R^{+}(r) \\
\left.\alpha_{j}^{h,+} \geq 0 \text { if }(h, j) \in R^{-}(r) \cup R^{0}(r)\right\} .
\end{array}\right.
\end{aligned}
$$

Given a particular bid-ask structure, equations (1)-(3) are also automatically satisfied. There is a solution $\left(t, q, \lambda^{-}, \lambda^{+}, \theta, c\right) \in \widetilde{P}(r)$ if and only if there is $\left(t, q, \alpha^{-}, \alpha^{+}, c\right)$, where $\left(\alpha^{-}, \alpha^{+}\right) \in$ $A^{-}(r) \times A^{+}(r)$, such that

$$
\begin{gathered}
\partial_{c_{0}^{h}} u^{h}(c)\left(q_{j}-k_{j}\right)-\sum_{s=1}^{S} \partial_{c_{s}^{h}} u^{h}(c) d_{s}^{j}+\left(\max \left\{0, \alpha_{j}^{h,-}\right\}\right)^{l}=0, \quad(h, j) \in H \times J, \\
\partial_{c_{0}^{h}} u^{h}(c)\left(q_{j}+k_{j}\right)-\sum_{s=1}^{S} \partial_{c_{s}^{h}} u^{h}(c) d_{s}^{j}-\left(\max \left\{0, \alpha_{j}^{h,+}\right\}\right)^{l}=0, \quad(h, j) \in H \times J, \\
c_{0}^{h}-e_{0}^{h}+\sum_{j \in J} q_{j}\left(\left(\max \left\{0,-\alpha_{j}^{h,+}\right\}\right)^{l}-\left(\max \left\{0,-\alpha_{j}^{h,-}\right\}\right)^{l}\right) \\
+\sum_{j \in J} k_{j}\left(\left(\max \left\{0,-\alpha_{j}^{h,-}\right\}\right)^{l}+\left(\max \left\{0,-\alpha_{j}^{h,+}\right\}\right)^{l}\right)=0, \quad h \in H, \\
c_{s}^{h}-e_{s}^{h}-\sum_{j \in J}\left(\left(\max \left\{0,-\alpha_{j}^{h,+}\right\}\right)^{l}-\left(\max \left\{0,-\alpha_{j}^{h,-}\right)^{l}\right\}\right) d_{s}^{j}=0, \quad h \in H, s \in S, \\
t \sum_{h \in H}\left(\left(\max \left\{0,-\alpha_{j}^{h,+}\right\}\right)^{l}-\left(\max \left\{0,-\alpha_{j}^{h,-}\right\}\right)^{l}\right) \\
+(1-t)\left(q_{j}^{0}-q_{j}\right)+t \frac{\varphi\left(\prod_{h \in H}\left(\max \left\{0, \alpha_{j}^{h,-}\right\}\right)^{l}\right)}{1+e^{q_{j}}}=0, \quad j \in J .
\end{gathered}
$$

The advantage of this reduced system is the absence of inequality constraints and the independence of the system of the sign vector $r$. We can use standard path-following methods to follow the path generated by this particular homotopy. Obviously the values of all variables along the path generated by this homotopy are identical to the values along the path generated by the theoretical homotopy. 


\section{Numerical Analysis: Examples}

In this section we report numerical results from an application of our algorithm to a few economies. First, we illustrate the algorithmic procedure via an example. Next we perform some comparative statics exercises and vary the level of transaction costs in some small economies.

We implemented our homotopy algorithm on a $450 \mathrm{MHz}$ PCPentiumII using the software package HOMPACK. This software package is a collection of FORTRAN 77 subroutines for solving systems of nonlinear equations using homotopy methods (Watson, Billups, and Morgan (1987)). From the three methods available in HOMPACK we selected the most robust path-following algorithm, which tracks the homotopy path by solving an ordinary differential equation. The starting point of the homotopy can be found using a standard nonlinear equation solver. We use a variation of the penalty approach of Schmedders (1998). We approximate the Jacobian of the homotopy function with a one-sided difference formula. When the path-following routine finds a solution we use a Newton routine to refine the solution to further reduce the error. In all our examples the maximum relative errors are of the order of magnitude of $10^{-10}$. The running time of the computer implementation of our algorithm is less than two seconds for the examples in the next sections.

\subsection{A First Example}

Consider an economy with $H=2$ agents, $S=4$ possible states in period $t=1$, and $J=2$ assets, called a bond and a stock. Both agents have identical von-Neumann-Morgenstern CRRA utility functions with identical uniform beliefs. That is, agent $i$ 's utility function equals:

$$
u^{i}(c)=\frac{c_{0}^{1-\gamma_{i}}}{1-\gamma_{i}}+\sum_{s=1}^{4} \frac{1}{4} \frac{c_{s}^{1-\gamma_{i}}}{1-\gamma_{i}}
$$

The two agents have coefficients of risk-aversion of $\gamma_{1}=5$ and $\gamma_{2}=1$, respectively. Both agents have an endowment of $e_{0}^{1}=e_{0}^{2}=1$ in period 0 . Agent 1 has an endowment (labor income) $e_{1}^{1}=(0.9,1.1,0.9,1.1)$ at date $t=1$; agent 2 has zero endowment at $t=1$, but he owns the entire stock paying dividends $d^{\text {st }}=(0.5,1.0,1.5,2.0)$. The stock is in unit net supply. (Agent 2's endowment at $t=0$ can be thought of as the stock's dividend at $t=0$.) The bond pays one unit in the second period regardless of the state of nature and is in zero net supply. Agents trading the bond and the stock have to pay identical transaction cost $k=k_{\mathrm{b}}=k_{\mathrm{st}}$ for both securities. In this first example we set $k=0.05$.

Using the practical homotopy of Section 5 we need to solve a system of $2 H J+H+H S+J=20$ equations with 21 unknowns. By solving equations (17) and (18) for the consumption variables and substituting the obtained values into the first-order conditions (15) and (16) we can reduce the system to the quite modest system with $2 H J+J=10$ equations and 11 unknowns. We use the expected payoffs of the two assets as values for the "starting" prices $q^{0}$, that is, $q_{\mathrm{b}}^{0}=1$ and $q_{\mathrm{st}}^{0}=1.25$. 


\section{[FIGURES 2 AND 3 ABOUT HERE]}

We depict the nature of the homotopy path in a few figures. Figures 2 and 3 show the change of agent 1's and agent 2's portfolio, respectively, as a function of the homotopy parameter. The behavior of the two portfolios is extremely different along the path. For small values of $t$ the first agent is long in the bond and short in the stock. This is not surprising, as the starting price system is based on the expected payoffs, and does not include a risk premium. The short position in the stock decreases quickly to zero as $t$ increases and the path of $\theta_{\mathrm{b}}^{1}$ exhibits a kink which in turn leads also to a kink in the path of $\theta_{\mathrm{st}}^{1}$. This type of behavior of the portfolio functions is typical; whenever one function exhibits a kink with a function value of zero then the other portfolio function also has a non-differentiability. However, these kinks in the first agent's portfolio functions do not affect the second agent's portfolio functions. Along the homotopy path no holding of the second agent hits zero for $t<1$ resulting in smooth portfolio functions. Note that equation (19) does not enforce

market clearing for $t<1$. Only as $t$ hits 1 the variable $\theta_{\mathrm{b}}^{2}$ decreases to zero. In equilibrium the bond market is closed. The equilibrium trade on the stock market equals a sale of 0.012 shares by agent 2 to agent 1 . Figure 4 displays the behavior of the asset prices along the homotopy path. Both price functions are smooth and are unaffected by the kinks in the portfolio functions of agent 1.

\section{[FIGURE 4 ABOUT HERE]}

\subsection{The Need for an Equilibrium Selection: An Example}

With this simple economy we can also show the importance of an equilibrium selection. Figure 5 shows the prices along the homotopy path for the homotopy without the term used for the equilibrium selection, that is, for the homotopy where equation (19) is replaced by the following equation:

$$
t \sum_{h \in H}\left(\left(\max \left\{0,-\alpha_{j}^{h,+}\right\}\right)^{l}-\left(\max \left\{0,-\alpha_{j}^{h,-}\right\}\right)^{l}\right)+(1-t)\left(q_{j}^{0}-q_{j}\right)=0, \quad j \in J .
$$

Until $t$ hits 1 the price paths are identical. But in equilibrium the bond is not traded due to the large transaction costs resulting in a continuum of equilibrium bond prices. If we don't force the homotopy to make an equilibrium selection then the path runs into this continuum and the homotopy exhibits a drop in rank at $t=1$ which causes numerical problems. (In this example the homotopy solver cannot find a unique stable solution at $t=1$ and instead finds many solutions with varying bond prices. Eventually the solver reports one of the found solutions and indicates a numerical problem.)

[FIGURE 5 ABOUT HERE] 


\subsection{Comparative Statics: A Two-Asset Model}

We perform a comparative statics analysis in our economy. We compute an equilibrium for various values of the transaction $\operatorname{costs} k$ in the interval [0,0.16]. Figure 6 shows the optimal portfolio of agent 1 as a function of the unit transaction cost $k$. Not surprisingly, the holdings decrease fast as $k$ increases. At around $k=0.04$ the bond market closes at which point the rate of decline of the stock holding changes considerably. Finally, as the unit transaction cost exceeds $k=0.157$ also the stock market closes. The economy has reached a no-trade equilibrium.

\section{[FIGURE 6 ABOUT HERE]}

Figure 7 shows the bond price at both the supply-perfect and the demand-perfect equilibrium as a function of the transaction cost $k$. Of course, as long as the bond is traded the bond prices at the two equilibria coincide. However, once the market closes the prices start to differ and their difference eventually becomes very substantial. Figure 8 shows the corresponding picture for the stock. The equilibrium stock price as a function of $k$ displays a kink at the $k$-value where the bond market closes.

\section{[FIGURES 7 AND 8 ABOUT HERE]}

\subsection{The Impact of Transaction Costs: A Three-Asset Model}

We extend the model to include a third security, a call option on the stock. The option is traded at time $t=0$ and has payoffs in period $t=1$ depending on the state of nature. The stock is not traded anymore at time $t=1$, so there is no endogenous uncertainty about the value of the stock and the option. In this simple two-period model the option's payoff is exogenously determined once the strike price has been set. Denoting the strike price by $K$ the return of the option in state $s \geq 1$ equals $\max \left(d_{s}^{\text {st }}-K, 0\right)$. We consider a strike price of $K=d_{2}^{\text {st }}=1.0$ leading to option payoffs of $d^{0}=(0,0,0.5,1.0)$ at date $t=1$. We examine the impact of identical real per unit transaction costs of $k=k_{\mathrm{b}}=k_{\mathrm{st}}=k_{\mathrm{o}}$ on the three securities.

\section{[FIGURE 9 ABOUT HERE]}

Figure 9 displays the portfolio of agent 1 as a function of the unit transaction cost $k$. Depending on the value of $k$ the portfolio positions of an agent can be vastly different. For very small transaction costs below 0.002 agent 1 is long in the option and short in both the bond and the stock. For approximately $0.002 \leq k \leq 0.0375$ the stock market is closed, and when $k \geq 0.0375$ agent 1 is long in the stock. The bond market closes at around $k=0.35$ and never opens again as $k$ increases further. Similarly, the option market closes for good around $k=0.047$. Once the option market closes the agents' portfolios are identical to those in the two-asset model. 
The figure shows that for some values of $k$ only the option market is open, for some other values agent 1 is short in the stock, and again for some other values he is long in the stock. Moreover, as $k$ increases the trading volume of an asset does not change monotonically. For example, as $k$ increases from 0 to about 0.002 the trading volume of the bond increases.

\section{[FIGURES 10,11,12 ABOUT HERE]}

Figures 10, 11, and 12 show the equilibrium prices of the bond, stock, and option, respectively, as a function of the unit transaction cost $k$. As discussed previously, whenever a market is closed there is a continuum of equilibrium prices bounded above by the price of the demand-perfect equilibrium and bounded below by the supply-perfect equilibrium price. Figure 11 shows how these two prices for the stock coincide for small values of $k$ when the agents trade the stock, then become different when the stock market is closed, and finally coincide again when the stock market reopens as $k$ exceeds 0.0375 .

\section{Conclusion}

We have presented the first algorithm for the computation of equilibria in finance economies with transaction costs on financial markets. Our homotopy algorithm is able to deal with the two major technical difficulties that are caused by transaction costs, namely the non-differentiabilities of agents' asset demand functions and the existence of robust examples of locally non-unique equilibria. The algorithm enables us to prove generic existence of an equilibrium, and we can also easily implement it using standard path-following software. We have computed the equilibria for

some small finance economies in order to illustrate the algorithm and the kind of comparative statics exercises it may serve to do. 


\section{Appendix: Proofs}

In this appendix we collect the proofs of all the results in our paper. We proceed in four steps. First, we present the proofs of the results that do not rely on transversality arguments, such as the properties of the no-arbitrage prices, equilibria, and demand functions. Secondly, we give a short overview of the theories of regular constraint sets and of manifolds with generalized boundaries, which we use heavily in the proofs of our main theorems. We motivate how we use this theory for our purpose. In the third section of this appendix we prove seven lemmas that are very important for our main results, since they allow us to use the mentioned theories. Finally, we complete the appendix with the proofs of the main theorems. In this final part of the Appendix, we first prove the theorems for the theoretical homotopy and subsequently we prove the results for the intuitive homotopy. This order is reverse to the development in the main part of the paper to simplify the arguments.

\section{A1: Proofs with no Transversality Arguments}

Proof of Proposition 2.2:

We define the matrix $M$ by

$$
M=\left[\begin{array}{cc}
(q-k)^{\top} & -(q+k)^{\top} \\
-A & A \\
I & 0 \\
0 & I
\end{array}\right],
$$

where $I$ is a $(J \times J)$-identity matrix and 0 a $(J \times J)$-zero matrix. By definition, a vector $q \in \mathbb{R}^{J}$ is a no-arbitrage price system if and only if for each $s=0, \ldots, S$, there is no solution $\theta^{s}=\left(\theta^{s,-}, \theta^{s,+}\right) \in \mathbb{R}^{J} \times \mathbb{R}^{J}$ to $M \theta^{s} \geq 0$ and $\left(M \theta^{s}\right)_{s}>0$. By the variant of Farkas' lemma given in Rockafellar (1970), Theorem 22.2, page 198, the latter condition is equivalent to: for every $s=0, \ldots, S$, there exists $\lambda^{s} \in \mathbb{R}_{+}^{1+S+2 J}$ with $\lambda_{s}^{s}>0$ and $\lambda^{s^{\top}} M=0$, which is the case if and only if there exists $\lambda \in \mathbb{R}_{++}^{1+S} \times \mathbb{R}_{+}^{2 J}$ such that $\lambda^{\top} M=0$. Now the theorem follows immediately after some elementary algebra.

Q.E.D.

\section{Proof of Proposition 2.3:}

The budget set $B^{h}(q)$ is a polyhedron, and so it is closed and convex. Extending the argument of Hens $\left(1991\right.$, p. 29) we next show that the budget set is also bounded. Let $l\left(q, \theta^{h,-}, \theta^{h,+}\right)=$ $\min \left\{(q-k) \cdot \theta^{h,-}-(q+k) \cdot \theta^{h,+}, d_{1}\left(\theta^{h,+}-\theta^{h,-}\right), \ldots, d_{S}\left(\theta^{h,+}-\theta^{h,-}\right)\right\}$ be the greatest loss incurred across all states from the portfolio $\left(\theta^{h,-}, \theta^{h,+}\right)$ at asset prices $q$. For $q \in Q$ and $\left(\theta^{h,-}, \theta^{h,+}\right) \neq 0$, $l\left(q, \theta^{h,-}, \theta^{h,+}\right)<0$ since $k>0$ and $A$ has full rank. The function $l\left(q, \theta^{h,-}, \theta^{h,+}\right)$ is homogeneous implying that $l\left(q, \theta^{h,-}, \theta^{h,+}\right) \leq l_{q}^{*}\left\|\left(\theta^{h,-}, \theta^{h,+}\right)\right\|$ where $l_{q}^{*}=\max _{\left\|\left(\theta^{h,-}, \theta^{h,+}\right)\right\|=1} l\left(q, \theta^{h,-}, \theta^{h,+}\right)<0$ exists due to the continuity of the function $l$. Now define $e^{* h}=\max \left\{e_{0}^{h}, e_{1}^{h}, \ldots, e_{S}^{h}\right\}$. Since $c^{h} \geq 0$ it follows that $e_{0}^{h} \geq(q+k) \cdot \theta^{h,+}-(q-k) \cdot \theta^{h,-}$ and $e_{s}^{h} \geq d_{s}\left(\theta^{h,-}-\theta^{h,+}\right), s=1, \ldots, S$ resulting for all $q \in Q$ in the inequalities $-e^{* h} \leq l\left(q, \theta^{h,-}, \theta^{h,+}\right) \leq l_{q}^{*}\left\|\left(\theta^{h,-}, \theta^{h,+}\right)\right\|$. Hence, $\left\|\left(\theta^{h,-}, \theta^{h,+}\right)\right\| \leq \frac{e^{* h}}{-l_{q}^{*}}$ 
for all $\left(\theta^{h,-}, \theta^{h,+}\right)$ such that there is a $c^{h}$ with $\left(\theta^{h,-}, \theta^{h,+}, c^{h}\right) \in B^{h}(q)$. Bounded portfolios only allow bounded consumption, so $\left\|c^{h}\right\|$ is bounded on $B^{h}(q)$.

Suppose $\left(\theta^{h,-}, \theta^{h,+}, c^{h}\right)$ is a solution to the agent's decision problem with $\theta^{h,-} \cdot \theta^{h,+}>0$. Then there is some asset $j^{\prime}$ such that $\theta_{j^{\prime}}^{h,-} \theta_{j^{\prime}}^{h,+}>0$. Without loss of generality it holds that $\theta_{j^{\prime}}^{h,-} \leq \theta_{j^{\prime}}^{h,+}$. Define the asset portfolio $\left(\widehat{\theta}^{h,-}, \widehat{\theta}^{h,+}\right)$ by $\widehat{\theta}_{j^{\prime}}^{h,-}=0, \widehat{\theta}_{j^{\prime}}^{h,+}=\theta_{j^{\prime}}^{h,+}-\theta_{j^{\prime}}^{h,-}$, and $\widehat{\theta}_{j}^{h,-}=\theta_{j}^{h,-}$, $\widehat{\theta}_{j}^{h,+}=\theta_{j}^{h,+}$, for $j \neq j^{\prime}$. Define the income stream $\widehat{c}^{h}$ by $\widehat{c}_{0}^{h}=c_{0}^{h}+2 k_{j^{\prime}} \theta_{j^{\prime}}^{h,-}$ and $\widehat{c}_{11}^{h}=c_{11}^{h}$. Clearly, $\left(\widehat{\theta}^{h,-}, \widehat{\theta}^{h,+}, \widehat{c}^{h}\right) \in B^{h}(q)$, and $c^{h}<\widehat{c}^{h}$, so $u^{h}\left(c^{h}\right)<u^{h}\left(\widehat{c}^{h}\right)$, a contradiction to the supposition that $\left(\theta^{h,-}, \theta^{h,+}, c^{h}\right)$ is a solution to the agent's decision problem. Consequently, $\theta^{h,-} \cdot \theta^{h,+}=0$.

Assumption A2 implies that agent $h$ has a unique utility maximizing consumption bundle $c^{h}$. From the full column rank of $A$ together with the property that at an optimal solution $\theta^{h,-} \cdot \theta^{h,+}=0$, it follows that the asset portfolio $\left(\theta^{h,-}, \theta^{h,+}\right)$ is uniquely determined as well.

Q.E.D.

\section{Proof of Proposition 3.2:}

Let $\left(\theta^{*}, q^{*}\right)$ be a competitive equilibrium of $\mathcal{E}$, inducing income streams used for consumption $c^{*}$. If for all $j \in J$ there exists $h \in H$ such that $\theta_{j}^{* h} \neq 0$ then $\left(\theta^{*}, q^{*}\right)$ is both a supply-perfect and a demand-perfect equilibrium and the proposition holds.

Suppose asset market $j \in J$ is such that $\theta_{j}^{* h}=0$ for all $h \in H$. We give the argument for the existence of a demand-perfect equilibrium that is allocationally equivalent to the competitive equilibrium; the argument for the existence of an allocationally equivalent supply-perfect equilibrium is similar. If $\min _{h \in H} \lambda_{j}^{h,-}\left(q^{*}\right)=0$, then we define $q_{j}^{\mathrm{d}}=q_{j}^{*}$. Otherwise, $\min _{h \in H} \lambda_{j}^{h,-}\left(q^{*}\right)=$ $\min _{h \in H}-\partial_{c_{0}^{* h}} u^{h}\left(c^{* h}\right)\left(q_{j}^{*}-k_{j}\right)+\sum_{s=1}^{S} \partial_{c_{s}^{h}} u^{h}\left(c^{* h}\right) d_{s}^{j}>0$. Since $\lambda_{j}^{h,-}(q)$ is a function that is linearly decreasing in $q_{j}$, we may define $q_{j}^{\mathrm{d}}$ unambiguously by

$$
\min _{h \in H}-\partial_{c_{0}^{* h}} u^{h}\left(c^{* h}\right)\left(q_{j}^{\mathrm{d}}-k_{j}\right)+\sum_{s=1}^{S} \partial_{c_{s}^{h}} u^{h}\left(c^{* h}\right) d_{s}^{j}=0 .
$$

If asset market $j \in J$ is such that $\theta_{j}^{* h} \neq 0$ for some $h \in H$, then we define $q_{j}^{\mathrm{d}}=q_{j}^{*}$.

Using the first-order conditions for the decision problem of household $h$, it is easily verified that $\theta^{* h}$ is an optimal asset portfolio at prices $q^{\mathrm{d}}$. For all asset markets $j$ for which $\theta_{j}^{* h}=0$ for all $h \in H$, it holds that $I_{j}^{-}\left(q^{\mathrm{d}}\right) \neq \emptyset$. It follows that $\left(\theta^{*}, q^{\mathrm{d}}\right)$ is a demand-perfect equilibrium. Q.E.D.

\section{Proof of Proposition 3.3:}

Consider a demand-perfect equilibrium induced by prices $q^{\mathrm{d}}$. It holds that $G\left(q^{\mathrm{d}}\right)=0$ and, for every asset $j$, either there is a household $h^{\prime}$ such that $g_{j}^{h^{\prime}}\left(q^{\mathrm{d}}\right) \neq 0$, or for all $h, g_{j}^{h}\left(q^{\mathrm{d}}\right)=0$ and there is a household $h^{\prime}$ such that $\lambda_{j}^{h^{\prime},-}\left(q^{\mathrm{d}}\right)=0$. In the first case it follows by the definition of a competitive equilibrium that without loss of generality $g_{j}^{h^{\prime}}\left(q^{\mathrm{d}}\right)<0$ and therefore $\lambda_{j}^{h^{\prime},-}\left(q^{\mathrm{d}}\right)=0$. In both cases it is then immediate that $\widetilde{G}\left(q^{\mathrm{d}}\right)=0$.

Consider a price system $q^{\mathrm{d}}$ such that $\widetilde{G}\left(q^{\mathrm{d}}\right)=0$. For every asset $j$, either $g_{j}^{h^{\prime}}\left(q^{\mathrm{d}}\right) \neq 0$ for some agent $h^{\prime}$, or $g_{j}^{h}\left(q^{\mathrm{d}}\right)=0$ for all agents $h=1, \ldots, H$. Since $\Lambda_{j}^{-}\left(q^{\mathrm{d}}\right) \geq 0, \varphi(0)=0$ and $\varphi^{\prime}>0$, it holds that $\varphi\left(\Lambda_{j}^{-}\left(q^{\mathrm{d}}\right)\right) \geq 0$. It follows that $g_{j}^{h^{\prime}}\left(q^{\mathrm{d}}\right)<0$ for some agent $h^{\prime}$ in the former case, so $\lambda_{j}^{h^{\prime},-}\left(q^{\mathrm{d}}\right)=0$ and $\Lambda_{j}^{-}\left(q^{\mathrm{d}}\right)=0$. Then it is immediate that $G_{j}\left(q^{\mathrm{d}}\right)=0$. In the latter case it holds 
that $G_{j}\left(q^{\mathrm{d}}\right)=0$, so $\Lambda_{j}^{-}\left(q^{\mathrm{d}}\right)=0$, which implies that $\lambda_{j}^{h^{\prime},-}\left(q^{\mathrm{d}}\right)=0$ for some agent $h^{\prime}$. Combining the two cases implies that $q^{\mathrm{d}}$ induces a demand-perfect equilibrium.

Q.E.D.

\section{Proof of Proposition 3.4:}

The necessary and sufficient first-order conditions that characterize $g^{h, r}$ on $Q(r)$ are given by

$$
\begin{aligned}
c_{0}^{h}-e_{0}^{h}+\sum_{\left\{j \mid r_{j}^{h}=-1\right\}} \theta_{j}^{h}\left(q_{j}-k_{j}\right)+\sum_{\left\{j \mid r_{j}^{h}=+1\right\}} \theta_{j}^{h}\left(q_{j}+k_{j}\right)=0, & \\
c_{s}^{h}-e_{s}^{h}-\sum_{\left\{j \mid r_{j}^{h} \in\{-1,+1\}\right\}} \theta_{j}^{h} d_{s}^{j}=0, & s \in S, \\
\partial_{c_{0}^{h}} u^{h}\left(c^{h}\right)\left(q_{j}-k_{j}\right)-\sum_{s=1}^{S} \partial_{c_{s}^{h}} u^{h}\left(c^{h}\right) d_{s}^{j}=0, & \text { if } r_{j}^{h}=-1, \\
\theta_{j}^{h}=0, & \text { if } r_{j}^{h}=0, \\
-\partial_{c_{0}^{h}} u^{h}\left(c^{h}\right)\left(q_{j}+k_{j}\right)+\sum_{s=1}^{S} \partial_{c_{s}^{h}} u^{h}\left(c^{h}\right) d_{s}^{j}=0, & \text { if } r_{j}^{h}=+1 .
\end{aligned}
$$

In fact, this set of conditions characterizes a demand function for assets on the set $Q$ of no-arbitrage prices, which satisfies that trade in assets $j$ for which $r_{j}^{h}=0$ is not permitted, the transaction costs $k_{j}$ are subtracted from the price $q_{j}$ when $r_{j}^{h}=-1$, and added to the price $q_{j}$ when $r_{j}^{h}=+1$. This

\begin{tabular}{|c|c|c|c|c|}
\hline$c^{h}$ & $\left(\theta_{j}^{h}\right)_{r_{j}^{h}=-1}$ & $\left(\theta_{j}^{h}\right)_{r_{j}^{h}=0}$ & $\left(\theta_{j}^{h}\right)_{r_{j}^{h}=+1}$ & \\
\hline \multirow{2}{*}{$I$} & $q_{j}-k_{j}$ & 0 & $q_{j}+k_{j}$ & \multirow{5}{*}{$\begin{array}{c}1 \\
S \\
r_{j}^{h}=-1 \\
r_{j}^{h}=0 \\
r_{j}^{h}=+1\end{array}$} \\
\hline & $-d^{j}$ & 0 & $-d^{j}$ & \\
\hline$\left(q_{j}-k_{j},-d^{j}\right) \partial^{2} u^{h}\left(c^{h}\right)$ & 0 & 0 & 0 & \\
\hline 0 & 0 & $I$ & 0 & \\
\hline$\left(q_{j}+k_{j},-d^{j^{\top}}\right) \partial^{2} u^{h}\left(c^{h}\right)$ & 0 & 0 & 0 & \\
\hline
\end{tabular}
function is an extension of $g^{h, r}$ to the open set $Q \supset Q(r)$. The implicit function theorem implies that $\left(\theta^{h}, c^{h}\right)$ is a twice differentiable function of $q$ if and only if the derivative with respect to $\left(\theta^{h}, c^{h}\right)$ of the left-hand side of the first-order conditions is nonsingular. This derivative is represented by the following matrix.

The variables above the matrix indicate those with respect to which the derivatives in the respective column have been taken. The numbers below the matrix show the number of columns. The notation $I$ denotes an identity matrix of appropriate dimension. Notice that the derivative doesn't have full rank if and only if there exists $\left(x_{1}, x_{2}\right) \in\left(\mathbb{R}^{1+S} \times \mathbb{R}^{\#\left\{j \mid r_{j}^{h}=-1\right.}\right.$ or $\left.\left.r_{j}^{h}=+1\right\}\right) \backslash\{0\}$ such that

$$
\begin{aligned}
& x_{1}^{\top}+x_{2}^{\top} B^{\top} \partial^{2} u^{h}=0, \\
& x_{1}^{\top} B=0,
\end{aligned}
$$

where $B$ is the matrix with columns $\left(q_{j}-k_{j},-d^{j^{\top}}\right)^{\top}$ for $j$ such that $r_{j}^{h}=-1$ or $r_{j}^{h}=+1$. Notice that $B$ has full column rank. Substituting for $x_{1}$ yields $-x_{2}^{\top} B^{\top} \partial^{2} u^{h} B=0$, which implies 
$-x_{2}^{\top} B^{\top} \partial^{2} u^{h} B x_{2}=0$. The full rank of $\partial^{2} u^{h}$ gives that $B x_{2}=0$, and so $x_{2}=0$ by the full column rank of $B$. Since $x_{1}^{\top}+x_{2}^{\top} B^{\top} \partial^{2} u^{h}=0$, it follows that $x_{1}=0$, contradicting that either $x_{1}$ or $x_{2}$ is not equal to zero. As a consequence, the derivative with respect to $\left(\theta^{h}, c^{h}\right)$ of the left-hand side of the first-order conditions is nonsingular. It follows that, for $r \in R, g^{h, r}$ is twice continuously differentiable for $h \in H$, which in turn implies the twice continuous differentiability of $G^{r}$.

For $r \in R$, if $r_{j}^{h}=-1$ for at least one household $h$, then $\Lambda_{j}^{-r}$ is identically equal to zero, so certainly twice continuous differentiable. Otherwise,

$$
\Lambda_{j}^{-r}(q)=\prod_{h=1}^{H}\left(-\partial_{c_{0}^{h}} u^{h}\left(c^{h}\right)\left(q_{j}-k_{j}\right)+\sum_{s=1}^{S} \partial_{c_{s}^{h}} u^{h}\left(c^{h}\right) d_{s}^{j}\right),
$$

where

$$
\begin{aligned}
c_{0}^{h} & =e_{0}^{h}-\sum_{\left\{j \mid r_{j}^{h}=-1\right\}} g_{j}^{h, r}(q)\left(q_{j}-k_{j}\right)-\sum_{\left\{j \mid r_{j}^{h}=+1\right\}} g_{j}^{h, r}(q)\left(q_{j}+k_{j}\right), \\
c_{s}^{h} & =e_{s}^{h}+\sum_{\left\{j \mid r_{j}^{h} \in\{-1,+1\}\right\}} g_{j}^{h, r}(q) d_{s}^{j}, \quad s \in S .
\end{aligned}
$$

The twice continuous differentiability of $g_{j}^{h, r}(q)$ implies that $\Lambda^{-r}$ is twice continuous differentiable.

Q.E.D.

\section{Proof of Proposition 3.5:}

Suppose the statement of the theorem is false. Then there is a subsequence, also denoted by $q^{n} \in Q$, such that $\left(g_{0}^{h}\left(q^{n}\right), g^{h}\left(q^{n}\right)\right) \rightarrow\left(\bar{\theta}_{0}^{h}, \bar{\theta}^{h}\right)$, where $\bar{\theta}_{0}^{h}=\bar{c}_{0}^{h}-e_{0}^{h}$ for some positive $\bar{c}_{0}^{h}$. Consider first the case where $q^{n} \rightarrow \bar{q} \in \partial Q$. For $\theta \in \mathbb{R}^{J}$, define $v^{h}(\theta)$ as the utility induced by portfolio $\theta$. We show that $\bar{\theta}^{h}$ maximizes $v^{h}$ when prices are $\bar{q}$. Since $v^{h}\left(g^{h}\left(q^{n}\right)\right) \geq u^{h}\left(e^{h}\right)$, it follows that the consumption bundle $\bar{c}^{h}$ generated by $\bar{\theta}^{h}$ is strictly positive. If not, then there exists $\widetilde{\theta} \in \mathbb{R}^{J}$ such that $v^{h}\left(\widetilde{\theta}^{h}\right)>v^{h}\left(\bar{\theta}^{h}\right)$, implying that the consumption bundle $\widetilde{c}^{h}$ induced by $\widetilde{\theta}^{h}$ is strictly positive, and in particular $\widetilde{c}_{0}^{h}>0$. It follows that $\widetilde{\theta}^{h}$ is affordable at prices $q^{n}$, for $n$ sufficiently large. By continuity, $v^{h}\left(\widetilde{\theta}^{h}\right)>v^{h}\left(g^{h}\left(q^{n}\right)\right)$, for $n$ sufficiently large, a contradiction to the optimality of $g^{h}\left(q^{n}\right)$. Consequently, $\bar{\theta}^{h}$ maximizes $v^{h}$ when prices are $\bar{q}$. Since $\bar{q}$ allows for arbitrage opportunities, this leads to a contradiction, and as a consequence the statement of the theorem can only be false if $\left\|q^{n}\right\| \rightarrow \infty$, the case we consider next.

Up to now we have normalized the price of date 0 consumption to be one. By homogeneity of degree 0 , demand for date 0 consumption and assets at prices $q_{0}^{n}:=1 /\left\|q^{n}\right\|$ for date 0 consumption, and prices $q^{n} /\left\|q^{n}\right\|$ for assets, denoted $\left(\widetilde{g}_{0}^{h}\left(1 /\left\|q^{n}\right\|, q^{n} /\left\|q^{n}\right\|\right), \widetilde{g}^{h}\left(1 /\left\|q^{n}\right\|, q^{n} /\left\|q^{n}\right\|\right)\right)$, equals $\left(g_{0}^{h}\left(q^{n}\right), g^{h}\left(q^{n}\right)\right)$. Suppose it is not the case that $\left\|\left(g_{0}^{h}\left(q^{n}\right), g^{h}\left(q^{n}\right)\right)\right\| \rightarrow \infty$. Then there is a subsequence, also denoted $q^{n}$, such that $\frac{q^{n}}{\left\|q^{n}\right\|} \rightarrow \bar{q}$ and $\left\|\left(g_{0}^{h}\left(q^{n}\right), g^{h}\left(q^{n}\right)\right)\right\|$ remains bounded, so $q^{n}$ can be chosen such that $\left(g_{0}^{h}\left(q^{n}\right), g^{h}\left(q^{n}\right)\right)$ converges to some $\left(\bar{\theta}_{0}^{h}, \bar{\theta}^{h}\right)$. Moreover, $\bar{\theta}^{h}$ is a utility maximizing portfolio at prices $\left(\bar{q}_{0}, \bar{q}\right)$. Suppose not, then there is $\widetilde{\theta}^{h}$ such that $v^{h}\left(\widetilde{\theta}^{h}\right)>v^{h}\left(\bar{\theta}^{h}\right)$, with $\widetilde{\theta}^{h}$ inducing a strictly positive consumption bundle $\widetilde{c}^{h}$, which implies that $\widetilde{\theta}^{h}$ is affordable at prices $\left(1 /\left\|q^{n}\right\|, q^{n} /\left\|q^{n}\right\|\right)$ for $n$ sufficiently large. Moreover, by continuity, $v^{h}\left(\widetilde{\theta}^{h}\right)>v^{h}\left(g^{h}\left(q^{n}\right)\right)$ for $n$ 
sufficiently large, which contradicts that $g^{h}\left(q^{n}\right)$ is the demand at $q^{n}$. Consequently, $\bar{\theta}^{h}$ maximizes utility at prices $\left(\bar{q}_{0}, \bar{q}\right)$. But this leads to a contradiction, since $\left\|q^{n}\right\| \rightarrow \infty$, so $1 /\left\|q^{n}\right\| \rightarrow 0$, so $\bar{q}_{0}=0$, and agents can choose unbounded date 0 consumption. Consequently, the first part of the proposition holds.

Since $q^{0} \in Q^{0}$, there is $\pi^{0} \in \mathbb{R}_{++}^{S}$ such that $q^{0}=\pi^{0^{\top}} A$. So, $\left(1, q^{0}\right) \cdot\left(g_{0}^{h}\left(q^{n}\right), g^{h}\left(q^{n}\right)\right)=$ $\left(1, \pi^{0^{\top}}\right) \cdot\left(g_{0}^{h}\left(q^{n}\right), A g^{h}\left(q^{n}\right)\right)$. Since $\left\|\left(g_{0}^{h}\left(q^{n}\right), g^{h}\left(q^{n}\right)\right)\right\| \rightarrow \infty$ and $A$ has full column rank, it follows that $\left\|\left(g_{0}^{h}\left(q^{n}\right), A g^{h}\left(q^{n}\right)\right)\right\| \rightarrow \infty$. Moreover, since $\left(g_{0}^{h}\left(q^{n}\right), A g^{h}\left(q^{n}\right)\right)$ is bounded below by $-e^{h}$, it follows that at least one component of $\left(g_{0}^{h}\left(q^{n}\right), A g^{h}\left(q^{n}\right)\right)$ converges to plus infinity, and therefore $\left(1, \pi^{0^{\top}}\right) \cdot\left(g_{0}^{h}\left(q^{n}\right), A g^{h}\left(q^{n}\right)\right) \rightarrow+\infty$.

Q.E.D.

Proof of Proposition 3.6:

By Proposition 3.5, $\left(1, q^{0}\right) \cdot\left(G_{0}\left(q^{n}\right), G\left(q^{n}\right)\right)=\sum_{h=1}^{H}\left(1, q^{0}\right) \cdot\left(g_{0}^{h}\left(q^{n}\right), g^{h}\left(q^{n}\right)\right) \rightarrow+\infty$. Q.E.D.

Proof of Proposition 4.1:

We define the function $f: \mathbb{R} \times Q(r) \rightarrow \mathbb{R} \times Q(r) \times \mathbb{R}^{H J} \times \mathbb{R}^{H J} \times \mathbb{R}^{H J} \times \mathbb{R}^{H(1+S)}$ by

$$
f(t, q)=\left(t, q, \lambda^{-}(q), \lambda^{+}(q), g(q), c(q)\right)
$$

where $c_{0}^{h}(q)=e_{0}^{h}-\sum_{j \in J} q_{j} g_{j}^{h}(q)+\sum_{j \in J_{h}^{-}(r)} k_{j} g_{j}^{h}(q)-\sum_{j \in J_{h}^{+}(r)} k_{j} g_{j}^{h}(q)$, and $c_{\mathbb{1}}^{h}=e_{\mathbb{1}}^{h}+A g^{h}(q)$. Then $(t, q) \in P(r)$ if and only if $f(t, q) \in \widetilde{P}(r)$. That the function $f$ is $C^{2}$ follows easily from Proposition 3.4. Obviously, $f^{-1}$ is $C^{\infty}$.

Q.E.D.

\section{A2: Regular Constraint Sets}

Since our convergence proof uses transversality theory, where the parameters to be perturbed are the initial endowments, we have the initial endowments entering the notation in the appendix. The proofs run very smoothly when we use the theory of regular constraint sets, see Herings (1997) for a first application of this theory in economics and an overview of the most relevant concepts.

For some $r \geq 1$ a subset $M$ of $\mathbb{R}^{k}$ is called a $C^{r} l$-dimensional manifold with generalized boundary (MGB), if for every $\bar{x} \in M$ there exists a local $C^{r}$ coordinate system of $\mathbb{R}^{k}$ around $\bar{x}$, i.e. a $C^{r}$ diffeomorphism $\zeta: U \rightarrow V$, where $U$ is an open subset of $\mathbb{R}^{k}$ containing $\bar{x}$ and $V$ is open in $\mathbb{R}^{k}$, and some $b(\bar{x}) \geq 0$ such that $\zeta(\bar{x})=0$ and $\zeta(U \cap M)$ equals

$$
\left\{y \in V \mid y_{1}=\cdots=y_{k-l}=0, y_{k-l+1} \geq 0, \ldots, y_{k-l+b(\bar{x})} \geq 0\right\} \text {. }
$$

If, for every element $\bar{x}$ of an MGB $M, b(\bar{x}) \leq 1$, then $M$ is called a manifold with boundary and it is easily shown that the set of elements $\bar{x}$ for which $b(\bar{x})=1$ is an $(l-1)$-dimensional manifold, called the boundary of $M$.

Let $I^{1}$ and $I^{2}$ be two finite index sets and let $\widetilde{g}_{i}, i \in I^{1}$, and $\widetilde{h}_{i}, i \in I^{2}$, be $C^{r}$ functions defined on some open subset $X$ of $\mathbb{R}^{k}$. We define

$$
M[\widetilde{g}, \widetilde{h}]=\left\{x \in X \mid \widetilde{g}_{i}(x)=0, i \in I^{1}, \widetilde{h}_{i}(x) \geq 0, i \in I^{2}\right\} .
$$


For $x \in X$, we define $I^{0}(x)=\left\{i \in I^{2} \mid h_{i}(x)=0\right\}$. If for every $\bar{x} \in M[\widetilde{g}, \widetilde{h}]$ it holds that

$$
\left\{\partial \widetilde{g}_{i}(\bar{x}), i \in I^{1}, \partial \widetilde{h}_{i}(\bar{x}), i \in I^{0}(\bar{x})\right\}
$$

is a set of independent vectors, then the equations $\widetilde{g}_{i}, \widetilde{h}_{i}$ are called a regular $C^{r}$ constraint system, and $M[\widetilde{g}, \widetilde{h}]$ is called a $C^{r}$ regular constraint set (RCS). In Jongen, et al. (1983, Lemma 3.1.2, Example 3.1.3) it is shown that every $C^{r} \operatorname{RCS}$ is a $\left(k-\left|I^{1}\right|\right)$-dimensional $C^{r}$ MGB with, for every $\bar{x} \in M[\widetilde{g}, \widetilde{h}], b(\bar{x})=\left|I^{0}(\bar{x})\right|$.

To show Theorem 3.7, we phrase the set $\widetilde{P}(r)$ as a regular constraint system. Typically, a given set $\widetilde{P}(r)$ can be represented by several such systems. Asset markets without trade will need a careful treatment. The set of asset markets without trade is denoted by

$$
J^{0}(r)=\cap_{h \in H} J_{h}^{0}(r) .
$$

Another special case occurs when only one agent is short in a certain asset market, whereas all other agents are inactive in that asset market. In equilibrium this is obviously impossible, but along the homotopy path such a situation may occur. The set of asset markets with one trader short and no other traders is denoted by $J_{-}^{1}(r)$,

$$
J_{-}^{1}(r)=\left\{j \in J \mid \exists h^{\prime} \in H, r_{j}^{h^{\prime}}=-1, r_{j}^{h}=0, h \neq h^{\prime}\right\} .
$$

When $J^{0}(r) \cup J_{-}^{1}(r) \neq \emptyset$, we need two different regular constraint systems, each representing a part of $\widetilde{P}(r)$ and together representing the entire set. The first regular constraint system is given by the system of equations (1)-(14). This system of equations is a regular constraint system for sign vectors $r$ such that $J^{0}(r) \cup J_{-}^{1}(r)=\emptyset$, and for values of $t<1$ when $J^{0}(r) \cup J_{-}^{1}(r) \neq \emptyset$.

It is not true that (1)-(14), when defined for all values of $t$, is a regular constraint system if $J^{0}(r) \cup J_{-}^{1}(r) \neq \emptyset$. Two problems occur at $t=1$. Suppose that there is an equilibrium without trade in asset market $j$. Then at $t=1$ the market clearing condition in (8) reduces to $\prod_{h=1}^{H} \lambda_{j}^{h,-}=0$, so for at least one household $\lambda_{j}^{h,-}=0$. But then necessarily two inequalities get binding at the same time, $t=1$ and $\lambda_{j}^{h,-}=0$. By counting the number of equations and unknowns, the matrix of derivatives of all equations and binding inequalities with respect to all endogenous variables cannot have full rank, so the augmented system is not a regular constraint system.

A similar problem occurs when along the homotopy path, for $t$ unequal but close to 1 , there is exactly one supplier in asset market $j$, say household $h^{\prime}$, whereas nobody demands asset $j$, and as a consequence $j \in J_{-}^{1}(r)$. Obviously, $\lambda_{j}^{h^{\prime},-}=0$, so, at $t=1$, the market clearing equation (8) implies $0=\sum_{h \in H} \theta_{j}^{h}=\theta_{j}^{h^{\prime}}$. Again, two inequalities get binding at the same time, $t=1$ and $\theta_{j}^{h^{\prime}}=0$, again implying that the augmented system is not a regular constraint system.

Although we have indicated that the system above is not a regular constraint system when $t$ is unrestricted and $J^{0}(r) \cup J_{-}^{1}(r) \neq \emptyset$, it remains to be shown that it is a regular constraint system when $t<1$ if $J^{0}(r) \cup J_{-}^{1}(r) \neq \emptyset$. We start out with a few useful lemmas first.

For $r \in R$, we define the open set $O^{r}$ as the set of all elements $\left(t, q, \lambda^{-}, \lambda^{+}, \theta, c, e\right) \in \mathbb{R} \times Q \times$ $\mathbb{R}^{H J} \times \mathbb{R}^{H J} \times \mathbb{R}^{H J} \times \mathbb{R}_{++}^{H(S+1)} \times \mathbb{R}_{++}^{H(S+1)}$ that satisfy the inequalities (9)-(14) with strict inequality. 
We define a function $\psi^{r}: O^{r} \rightarrow \mathbb{R}^{H(S+1)+3 H J+J}$ by associating the left-hand side of equations (1)(8) to $\psi^{r}\left(t, q, \lambda^{-}, \lambda^{+}, \theta, c, e\right)$. For $e \in E$, the function $\psi^{r, e}$ is defined by $\psi^{r, e}(\cdot)=\psi^{r}(\cdot, e)$.

\section{A3: Transversality Arguments}

We state and prove seven lemmas that are crucial for the latter proofs of our main results. These lemmas state all the relevant properties of our homotopy paths and loops so that we can apply the theories of regular constraint sets and of manifolds with generalized boundaries. Intuitively, the first three lemmas provide the tools for the typical genericity arguments in homotopy applications. The first lemma implies that (generically) the set of zeros of our homotopy is one-dimensional, the second one implies that boundaries are hit transversely, and the third one shows that never two boundaries are hit simultaneously. Unfortunately, due to the described problems at $t=1$ these results do not suffice for our problem. Therefore, we need four additional lemmas in order to deal with the special case at $t=1$. As we will see, the intuition for the situation at $t=1$ will be just as in the standard situation.

We begin with Lemma A.1 showing that the set of solutions to (1)-(8), with the inequality constraints satisfied with strict inequality, is a 1-dimensional manifold.

Lemma A.1: There is a subset $E^{1}$ of $E$ of full Lebesgue measure such that for all $e \in E^{1}$, for all $r \in R, \psi^{r, e}$ 历 $\{0\}$.

Proof: Fix some $r \in R$. First, it is shown that $\psi^{r} 历\{0\}$. Let $\bar{\xi}=\left(\bar{t}, \bar{q}, \bar{\lambda}^{-}, \bar{\lambda}^{+}, \bar{\theta}, \bar{c}, \bar{e}\right)$ be such that $\psi^{r}(\bar{\xi})=0$. The matrix of partial derivatives of $\psi^{r}$ evaluated at $\bar{\xi}$ is denoted by $\bar{M}$ and is given in Table 3. It has to be shown that the rows of $\bar{M}$ are independent. This is done by proving that $y^{\top} \bar{M}=0$ implies $y=0$.

The matrix $\bar{M}$ is subdivided into eight parts in Table 3 , and the components of $y$ will be denoted accordingly, so $y=\left(y_{1}, y_{2}, \ldots, y_{8}\right)$, where $y_{1}=\left(y_{1, h, j}\right)_{(h, j) \in R^{-}(r)}, y_{2}=\left(y_{2, h, j}\right)_{(h, j) \in R^{+}(r)}, y_{3}=$ $\left(y_{3, h, j}\right)_{(h, j) \in R^{0}(r)}, y_{4}=\left(y_{4, h}\right)_{h \in H}, y_{5}=\left(y_{5, h, s}\right)_{(h, s) \in H \times S}, y_{6}=\left(y_{6, h, j}\right)_{(h, j) \in H \times J}, y_{7}=\left(y_{7, h, j}\right)_{(h, j) \in H \times J}$, and $y_{8}=\left(y_{8, j}\right)_{j \in J}$. The last column in the table gives the number of rows in each one of the eight parts. The last row in the table indicates the number of columns in the corresponding part. We order $\partial_{\theta_{j}^{h}}$ first by $h$ and then by $j$. We order $\partial_{c_{s}^{h}}$ first by $h$ and then by $s$. For $\partial_{e}$, we start with $\partial_{e_{0}}$ and continue with $\partial_{e_{\mathbb{1}}}$. Parts in the table indicated by $\star$ are irrelevant for our proof. An identity matrix of appropriate dimension is denoted by $I$, and a matrix of zeroes by 0 . Row $(h, j),(h, j) \in R^{-}(r)$, of the matrix $E^{-}$contains exactly one 1 , corresponding to the derivative with respect to $\lambda_{j}^{h,-}$. Row $(h, j),(h, j) \in R^{+}(r)$, of the matrix $E^{+}$contains exactly one 1 , corresponding to the derivative with respect to $\lambda_{j}^{h,+}$. Row $(h, j),(h, j) \in R^{0}(r)$, of the matrix $E^{0}$ contains exactly one 1 , corresponding to the derivative with respect to $\theta_{j}^{h}$. The vector $\bar{\alpha} \in \mathbb{R}^{J}$ is defined by $\bar{\alpha}_{j}=\sum_{h=1}^{H} \bar{\theta}_{j}^{h}-q_{j}^{0}+\bar{q}_{j}+\varphi\left(\prod_{h=1}^{H} \bar{\lambda}_{j}^{h,-}\right) /\left(1+e^{q_{j}}\right)$. The matrix $\Lambda^{h^{\prime}}$ is a diagonal matrix with $\Lambda_{j j}^{h^{\prime}}=\bar{t} \varphi^{\prime}\left(\prod_{h=1}^{H} \bar{\lambda}_{j}^{h,-}\right) \prod_{h \neq h^{\prime}} \bar{\lambda}_{j}^{h,-} /\left(1+e^{q_{j}}\right)$. The matrix $T$ is a diagonal matrix with 


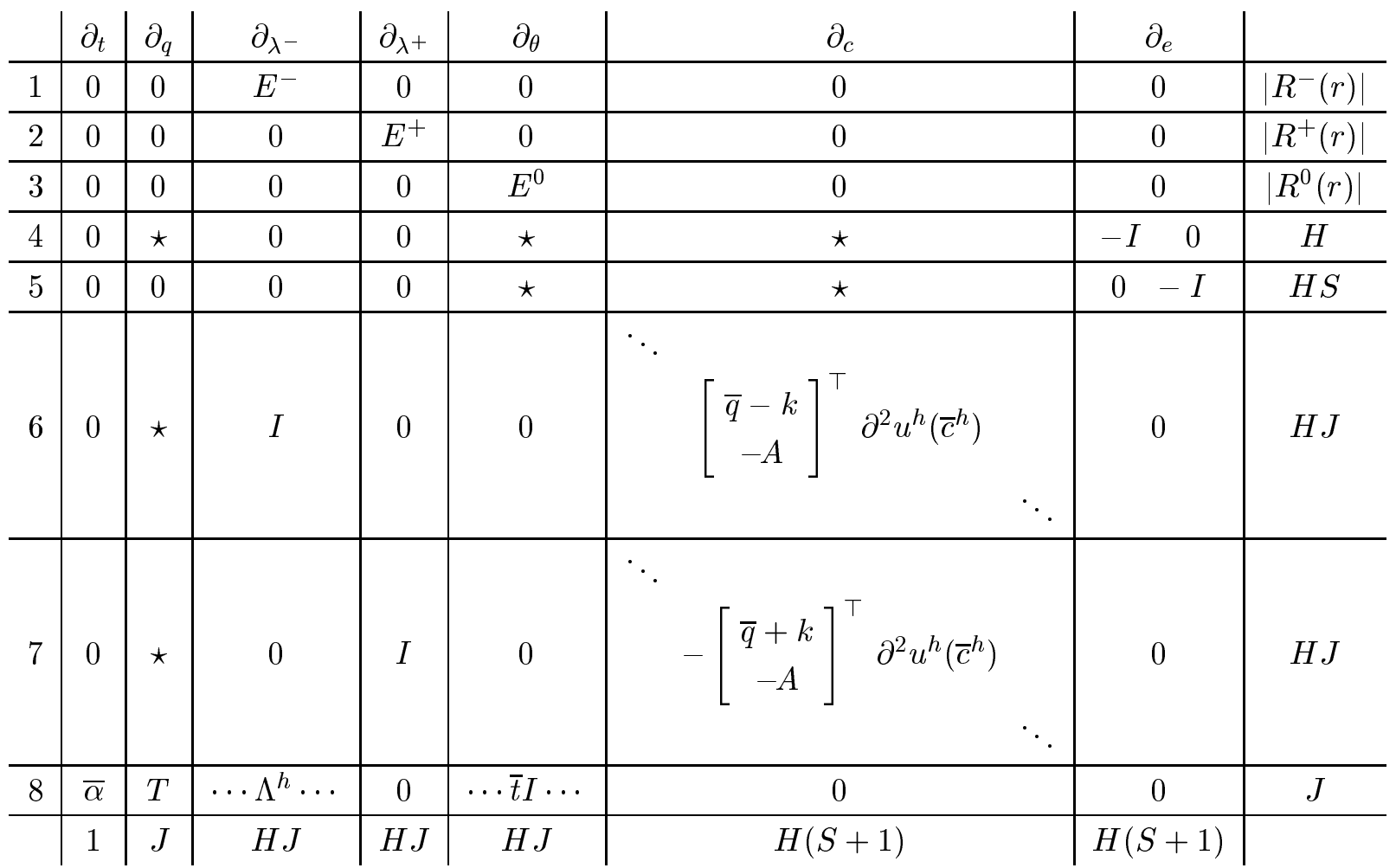

TABle 3 . The matrix $\bar{M}$.

$T_{j j}=(\bar{t}-1)-\bar{t} \varphi\left(\prod_{h=1}^{H} \bar{\lambda}_{j}^{h,-}\right) e^{\bar{q}_{j}} /\left(1+e^{\bar{q}_{j}}\right)^{2}$.

Let $y \in \mathbb{R}^{H(S+1)+3 H J+J}$ satisfy $y^{\top} \bar{M}=0$. From Table 3 it is immediate that $y_{4}=0$ and $y_{5}=0$; simply consider $\partial_{e} \psi^{r}(\bar{\xi})$. It also holds that

$$
y^{\top} \partial_{c^{h}} \psi^{r}(\bar{\xi})=\sum_{j=1}^{J} y_{6, h, j}\left(\bar{q}_{j}-k_{j},-d^{j^{\top}}\right) \partial^{2} u^{h}\left(\bar{c}^{h}\right)-\sum_{j=1}^{J} y_{7, h, j}\left(\bar{q}_{j}+k_{j},-d^{j^{\top}}\right) \partial^{2} u^{h}\left(\bar{c}^{h}\right) .
$$

For $j \in J_{h}^{-}(r) \cup J_{h}^{0}(r)$ it holds that $0=y^{\top} \partial_{\lambda_{j}^{h,+}} \psi^{r}(\bar{\xi})=y_{7, h, j}$. Consider some $j \in J_{h}^{+}(r)$. Since $\bar{t}>0$ and $0=y^{\top} \partial_{\theta_{j}^{h}} \psi^{r}(\bar{\xi})=\bar{t} y_{8, j}$, it holds that $y_{8, j}=0$. Then it follows that $0=y^{\top} \partial_{\lambda_{j}^{h,-}} \psi^{r}(\bar{\xi})=y_{6, h, j}$ for $j \in J_{h}^{+}(r)$. Equation (25) reduces to

$$
y^{\top} \partial_{c^{h}} \psi^{r}(\bar{\xi})=\sum_{j \in J_{h}^{-}(r) \cup J_{h}^{0}(r)} y_{6, h, j}\left(\bar{q}_{j}-k_{j},-d^{j^{\top}}\right) \partial^{2} u^{h}\left(\bar{c}^{h}\right)-\sum_{j \in J_{h}^{+}(r)} y_{7, h, j}\left(\bar{q}_{j}+k_{j},-d^{j^{\top}}\right) \partial^{2} u^{h}\left(\bar{c}^{h}\right) .
$$

Since the rank of $A$ equals $J$, the matrix

$$
\left[\left(\begin{array}{c}
\bar{q}_{j}-k_{j} \\
-d^{j}
\end{array}\right)_{j \in J_{h}^{-}(r) \cup J_{h}^{0}(r)},-\left(\begin{array}{c}
\bar{q}_{j}+k_{j} \\
-d^{j}
\end{array}\right)_{j \in J_{h}^{+}(r)}\right]
$$

has rank $J$. The rank of $\partial^{2} u^{h}\left(\bar{c}^{h}\right)$ equals $S+1$ implying that $y_{6, h, j}=0$ for $j \in J_{h}^{-}(r) \cup J_{h}^{0}(r)$ and $y_{7, h, j}=0$ for $j \in J_{h}^{+}(r)$. Consequently, $y_{6}=0$ and $y_{7}=0$. 
Since $\bar{t}<1$ by definition of $\psi^{r}$ and $0=y^{\top} \partial_{q_{j}} \psi^{r}(\bar{\xi})=\left[(\bar{t}-1)-\bar{t} \varphi\left(\prod_{h=1}^{H} \bar{\lambda}_{j}^{h,-}\right) e^{\bar{q}_{j}} /\left(1+e^{\bar{q}_{j}}\right)^{2}\right] y_{8, j}$, it follows that $y_{8, j}=0$, for $j \in J$.

Now it follows from $\partial_{\lambda^{-}} \psi^{r}(\bar{\xi}), \partial_{\lambda^{+}} \psi^{r}(\bar{\xi})$, and $\partial_{\theta} \psi^{r}(\bar{\xi})$, respectively, that $y_{1}=0, y_{2}=0$, and $y_{3}=0$.

We conclude that $\psi^{r} 历\{0\}$. The lemma then follows directly from the parametric transversality theorem as stated for instance in Mas-Colell (1985), I.2.2, page 45.

Q.E.D.

We denote the set of indices corresponding to the inequalities in (9)-(14) by $I$, with generic element $i$. For $r \in R$ with $J^{0}(r) \cup J_{-}^{1}(r)=\emptyset$ and $i \in I$, we define the open set $O_{i}^{r}$ as the set of all elements $\left(t, q, \lambda^{-}, \lambda^{+}, \theta, c, e\right) \in \mathbb{R} \times Q \times \mathbb{R}^{3 H J} \times \mathbb{R}_{++}^{2 H(S+1)}$ that satisfy the inequalities (9)-(14) with strict inequality, with the exception of the inequality related to $i$, which is not taken into account. For $r \in R$ with $J^{0}(r) \cup J_{-}^{1}(r) \neq \emptyset$ and $i \in I$ the definition is similar, but now $t$ is restricted to $(-\infty, 1)$, and $i$ never corresponds to inequality (14).

For $r \in R$ and $i \in I$, we define a function $\psi_{i}^{r}: O_{i}^{r} \rightarrow \mathbb{R}^{H(S+1)+3 H J+J+1}$, by associating the left-hand side of equations (1)-(8) to $\psi_{i}^{r}\left(t, q, \lambda^{-}, \lambda^{+}, \theta, c, e\right)$, and the left-hand side of exactly one inequality, indexed by $i$, in $(9)-(14)^{7}$. For $e \in E$, the function $\psi_{i}^{r, e}$ is defined by $\psi_{i}^{r, e}(\cdot)=\psi_{i}^{r}(\cdot, e)$.

The next lemma, Lemma A.2, serves to show that the set of solutions to (1)-(8) and any binding inequality $i$, is a 0 -dimensional manifold. Moreover, it shows that the set of solutions to (1)-(8) intersects the manifold generated as the solutions to inequality $i$ in a transversal way.

Lemma A.2: There is a subset $E^{2}$ of $E$ of full Lebesgue measure such that for all $e \in E^{2}$, $r \in R$, and $i \in I, \psi_{i}^{r, e}$ 历 $\{0\}$.

Proof: Fix some $r \in R$ and some $i \in I$. We start out by showing that $\psi_{i}^{r} 历\{0\}$. Let $\bar{\xi}=$ $\left(\bar{t}, \bar{q}, \bar{\lambda}^{-}, \bar{\lambda}^{+}, \bar{\theta}, \bar{c}, \bar{e}\right)$ be such that $\psi_{i}^{r}(\bar{\xi})=0$. It has to be shown that the rows of the matrix of partial derivatives of $\psi^{r}$ evaluated at $\bar{\xi}$, denoted by $\bar{M}_{i}$, are independent. Let $y \in \mathbb{R}^{H(S+1)+3 H J+J+1}$ satisfy $y^{\top} \bar{M}_{i}=0$. We index $y$ as before, and let $y_{9}$ be the component of $y$ corresponding to inequality $i$. Three cases have to be distinguished, depending on which inequality $i$ has been chosen.

CASE 1. The index $i$ corresponds to one of the inequalities in (9)-(10).

If $\lambda_{j^{\prime}}^{h^{\prime},-}=0$ is added for some $\left(h^{\prime}, j^{\prime}\right) \in R^{0}(r)$, then by the proof of Lemma A.1 it follows that $y_{1}=\cdots=y_{8}=0$. It is shown that $y_{9}=0$ by considering $\partial_{\lambda_{j^{\prime}}^{h^{\prime},-}} \psi^{r}(\bar{\xi})$.

If $\lambda_{j^{\prime}}^{h^{\prime},+}=0$ is added for some $\left(h^{\prime}, j^{\prime}\right) \in R^{0}(r)$, then it follows from the proof of Lemma A.1 that $y_{4}=0, y_{5}=0, y_{6, h, j}=0,(h, j) \in R^{+}(r)$, and $y_{7, h, j}=0,(h, j) \in R^{-}(r) \cup R^{0}(r) \backslash\left\{\left(h^{\prime}, j^{\prime}\right)\right\}$. For $h \neq h^{\prime}$, it follows that $y_{6, h, \cdot}=0$ and $y_{7, h, \cdot}=0$ as before. The proof for $h^{\prime}$ is more complicated. We claim first that $\operatorname{rank}(A)=J$ implies that the matrix

$$
B=\left[\left(\begin{array}{c}
\bar{q}_{j}-k_{j} \\
-d^{j}
\end{array}\right)_{j \in J_{h^{\prime}}^{-}(r) \cup J_{h^{\prime}}^{0}(r)},-\left(\begin{array}{c}
\bar{q}_{j}+k_{j} \\
-d^{j}
\end{array}\right)_{j \in J_{h^{\prime}}^{+}(r) \cup\left\{j^{\prime}\right\}}\right]
$$

\footnotetext{
${ }^{7}$ Again, when $J^{0}(r) \cup J_{-}^{1}(r) \neq \emptyset$, then $i$ is never chosen to correspond to inequality (14).
} 
has rank $J+1$. Indeed, suppose we partition $B$ in three parts corresponding to indices in $J \backslash\left\{j^{\prime}\right\}$, the column corresponding to $j^{\prime}$ where transaction costs are subtracted from the price, and the column corresponding to $j^{\prime}$ where transaction costs are added. Then $\left[B_{1}, B_{2}, B_{3}\right]\left(x_{1}^{\top}, x_{2}, x_{3}\right)^{\top}=0$ implies $x_{1}=0$ and $x_{2}-x_{3}=0$, because $A$ has rank $J$. Next,

$$
\left(\bar{q}_{j^{\prime}}-k_{j^{\prime}}\right) x_{2}-\left(\bar{q}_{j^{\prime}}+k_{j^{\prime}}\right) x_{3}=\bar{q}_{j^{\prime}}\left(x_{2}-x_{3}\right)-k_{j^{\prime}}\left(x_{2}+x_{3}\right)=-k_{j^{\prime}}\left(x_{2}+x_{3}\right)=0,
$$

so $x_{2}=x_{3}=0$, completing the proof of the claim. Since the matrix $B$ has rank $J+1$, it follows from $y^{\top} \partial_{c^{h^{\prime}}} \psi^{r}(\bar{\xi})=0$ that $y_{6, h^{\prime},}=0$ and $y_{7, h^{\prime},}=0$. The remainder of the proof is as before.

CASE 2. The index $i$ corresponds to one of the inequalities in (11)-(12).

If $\theta_{j^{\prime}}^{h^{\prime}}=0$ is added for some $\left(h^{\prime}, j^{\prime}\right) \in R^{-}(r)$, then the proof of Lemma A.1 can be used to show that $y_{1}=\cdots=y_{8}=0$. Furthermore, $0=y^{\top} \partial_{\theta_{j^{\prime}}^{h^{\prime}}} \psi_{i}^{r}(\bar{\xi})=y_{9}$.

If $\theta_{j^{\prime}}^{h^{\prime}}=0$ is added for some $\left(h^{\prime}, j^{\prime}\right) \in R^{+}(r)$, then it follows as in the proof of Lemma A.1 that $y_{4}=0, y_{5}=0, y_{6, h, j}=0,(h, j) \in R^{+}(r)$, and $y_{7, h, j}=0,(h, j) \in R^{-}(r) \cup R^{0}(r) \backslash\left\{\left(h^{\prime}, j^{\prime}\right)\right\}$. For $h \neq h^{\prime}$, it follows that $y_{6, h,}=0$ and $y_{7, h, \cdot}=0$ by considering $\partial_{c^{h}} \psi_{i}^{r}(\bar{\xi})$. For $h^{\prime}$ this follows as well, since the matrix $B$ has rank $J+1$. The remainder of the proof is as before.

CASE 3. The index $i$ corresponds to one of the inequalities in (13)-(14).

If $t=0$ is added, then it follows as in the proof of Lemma A. 1 that $y_{4}=0, y_{5}=0$, and $y_{7, h, j}=0$, $(h, j) \in R^{-}(r) \cup R^{0}(r)$. Since $\bar{t}=0, \Lambda^{h}=0$ for all $h$ and $0=y^{\top} \partial_{\lambda_{j}^{h,-}} \psi_{i}^{r}(\bar{\xi})=y_{6, h, j},(h, j) \in R^{+}(r)$. The remainder of the proof is as before.

If $t=1$ is added, then it follows as in the proof of Lemma A.1 that $y_{4}=\cdots=y_{7}=0$. Since this equality is only added when $J^{0}(r) \cup J_{-}^{1}(r)=\emptyset$, for each $j$ there is $h(j)$ such that $r_{j}^{h(j)} \neq 0$. Then $0=y^{\top} \partial_{\theta_{j}^{h(j)}} \psi_{i}^{r}(\bar{\xi})=y_{8, j}$. The remainder of the proof is standard.

We conclude that $\psi_{i}^{r} \pitchfork\{0\}$. The lemma now follows from the parametric transversality theorem.

Q.E.D.

For $r \in R$ with $J^{0}(r) \cup J_{-}^{1}(r)=\emptyset$, we define the open set $\widetilde{O}^{r}$ by $\widetilde{O}^{r}=\mathbb{R} \times Q \times \mathbb{R}^{3 H J} \times \mathbb{R}_{++}^{2 H(S+1)}$, and for $r \in R$ with $J^{0}(r) \cup J_{-}^{1}(r) \neq \emptyset$, we define $\widetilde{O}^{r}=(-\infty, 1) \times Q \times \mathbb{R}^{3 H J} \times \mathbb{R}_{++}^{2 H(S+1)}$. We define a function $\psi_{i^{1}, i^{2}}^{r}: \widetilde{O}^{r} \rightarrow \mathbb{R}^{H(S+1)+3 H J+J+2}$, by associating the left-hand side of equations (1)-(8) to $\psi_{i^{1}, i^{2}}^{r}\left(t, q, \lambda^{-}, \lambda^{+}, \theta, c, e\right)$, and the left-hand side of exactly two different inequalities, indexed by $i^{1}, i^{2}$, in $(9)-(14)^{8}$. For $e \in E$, the function $\psi_{i^{1}, i^{2}}^{r, e}$ is defined by $\psi_{i^{1}, i^{2}}^{r, e}(\cdot)=\psi_{i^{1}, i^{2}}^{r}(\cdot, e)$.

The purpose of Lemma A.3 is to show it cannot occur that two inequalities become binding at the same time.

LEMma A.3: There is a subset $E^{3}$ of $E$ of full Lebesgue measure such that for all $e \in E^{3}$, $r \in R, i^{1}, i^{2} \in I, i^{1} \neq i^{2}, \psi_{i^{1}, i^{2}}^{r, e}$ 历 $\{0\}$.

Proof: Fix some $r \in R$ and some $i^{1}, i^{2} \in I$ with $i^{1} \neq i^{2}$. We start out by showing that $\psi_{i^{1}, i^{2}}^{r} 历\{0\}$. Six cases have to be distinguished, depending on which inequalities $i^{1}, i^{2}$ have been chosen.

\footnotetext{
${ }^{8}$ Again, when $J^{0}(r) \cup J_{-}^{1}(r) \neq \emptyset$, none of the indices is taken to correspond to $(14)$.
} 
CASE 1. The indices $i^{1}, i^{2}$ both correspond to inequalities in (9)-(10). The proof can be done by using the techniques of Lemma A.2, unless the equations added are $\lambda_{j^{1}}^{h^{1},+}=\lambda_{j^{2}}^{h^{2},+}=0$ and $h^{1}=h^{2}$. We consider the latter case in more detail.

That $y_{4}=0$ and $y_{5}=0$ follows as before.

If $\bar{t} \prod_{h \neq h^{1}} \bar{\lambda}_{j^{1}}^{h,-}=0$, then $0=y^{\top} \partial_{\lambda_{j^{1}}^{h^{1},-}} \psi_{i^{1}, i^{2}}^{r}(\bar{\xi})=y_{6, h^{1}, j^{1}}$, and the remainder of the proof follows as in Lemma A.2. Suppose $\bar{t} \prod_{h \neq h^{1}} \bar{\lambda}_{j^{1}}^{h,-} \neq 0$. This implies in particular that $\bar{t} \neq 0$.

If $\exists h \in H, r_{j^{1}}^{h} \neq 0$, then $0=y^{\top} \partial_{\theta_{j^{1}}} \psi_{i^{1}, i^{2}}^{r}(\bar{\xi})=\bar{t} y_{8, j^{1}}$, so $y_{8, j^{1}}=0$, and $y_{6, h^{1}, j^{1}}=0$ as well, and the remainder of the proof follows easily. Suppose $r_{j^{1}}^{h}=0, \forall h \in H$. Then $j^{1} \in J^{0}(r)$ and it follows that $\bar{t}<1$ by definition of $\psi_{i^{1}, i^{2}}^{r}$.

If $h^{1}$ is not the only household in the economy, say there exists a household $h^{3}$ other than $h^{1}$, then it is straightforward to show that $y_{6, h^{3}, .}=0$ and $y_{7, h^{3}, .}=0$. Since $\bar{\lambda}_{j^{1}}^{h^{1},+}=0$, it follows from equations (6) and (7) that $\bar{\lambda}_{j^{1}}^{h^{1},-} \neq 0$, and since $\bar{t} \prod_{h \neq h^{1}} \bar{\lambda}_{j^{1}}^{h,-} \neq 0$, it follows that $\bar{t} \prod_{h \neq h^{3}} \bar{\lambda}_{j^{1}}^{h,-} \neq 0$. So $0=y^{\top} \partial_{\lambda_{j^{1}}^{h^{3},-}} \psi_{i^{1}, i^{2}}^{r}(\bar{\xi})=\left[\bar{t} \varphi^{\prime}\left(\prod_{h=1}^{H} \bar{\lambda}_{j^{1}}^{h,-}\right) \prod_{h \neq h^{3}} \bar{\lambda}_{j^{1}}^{h,-} /\left(1+e^{q_{j}}\right)\right] y_{8, j^{1}}$, which implies that $y_{8, j^{1}}=0$, and it follows that $y_{6, h^{1}, j^{1}}=0$ as well. The remainder of the proof is straightforward.

Suppose next that $h^{1}$ is the only household in the economy. From $0=y^{\top} \partial_{c^{h^{1}}} \psi_{i^{1}, i^{2}}^{r}(\bar{\xi})$ it follows that

$$
\sum_{j=1}^{J} y_{6, h^{1}, j}\left(\bar{q}_{j}-k_{j},-d^{j^{\top}}\right) \partial^{2} u^{h^{1}}\left(\bar{c}^{h^{1}}\right)-\sum_{j=1}^{J} y_{7, h^{1}, j}\left(\bar{q}_{j}+k_{j},-d^{j^{\top}}\right) \partial^{2} u^{h^{1}}\left(\bar{c}^{h^{1}}\right)=0 .
$$

Since $\partial^{2} u^{h^{1}}\left(\bar{c}^{h^{1}}\right)$ has full rank, this implies that

$$
\sum_{j=1}^{J} y_{6, h^{1}, j}\left(\bar{q}_{j}-k_{j},-d^{j^{\top}}\right)-\sum_{j=1}^{J} y_{7, h^{1}, j}\left(\bar{q}_{j}+k_{j},-d^{j^{\top}}\right)=0
$$

and in particular

$$
\sum_{j=1}^{J}\left(y_{6, h^{1}, j}-y_{7, h^{1}, j}\right) d^{j^{\top}}=0 \text {. }
$$

Since $A$ has full column rank, this implies that $y_{6, h^{1}, \cdot}-y_{7, h^{1}, \cdot}=0$. Moreover,

$$
0=y^{\top} \partial_{q} \psi_{i^{1}, i^{2}}^{r}(\bar{\xi})=\partial_{c_{0}^{h^{1}}} u^{h^{1}}\left(\bar{c}^{h^{1}}\right) y_{6, h^{1}, \cdot}-\partial_{c_{0}^{h^{1}}} u^{h^{1}}\left(\bar{c}^{h^{1}}\right) y_{7, h^{1}, \cdot}+T y_{8, \cdot}=T y_{8, \cdot}
$$

Since $T$ has full rank, it follows that $y_{8, .}=0$. For $j \in J_{h^{1}}^{0}(r) \cup J_{h^{1}}^{+}(r)$, we find that $y_{6, h^{1}, j}=y_{7, h^{1}, j}=0$ by considering $\partial_{\lambda^{h^{1},-}} \psi_{i^{1}, i^{2}}^{r}(\bar{\xi})$. Using that $j \neq j^{1}$ and $j \neq j^{2}$, we find for $j \in J_{h^{1}}^{-}(r)$ that

$$
0=y^{\top} \partial_{\lambda_{j}^{h^{1},+}} \psi_{i^{1}, i^{2}}^{r}(\bar{\xi})=y_{7, h^{1}, j}=y_{6, h^{1}, j} .
$$

The remainder of the proof is straightforward.

CASE 2. The index $i^{1}$ corresponds to an inequality in (9)-(10) and the index $i^{2}$ to an inequality in (11)-(12). The proof is similar to the one of Lemma A.2, unless the equations added are 
$\lambda_{j^{1}}^{h^{1},+}=0$, for some $\left(h^{1}, j^{1}\right) \in R^{0}(r), \theta_{j^{2}}^{h^{2}}=0$, for some $\left(h^{2}, j^{2}\right) \in R^{+}(r)$, and $h^{1}=h^{2}$. But then the proof is similar to the one of Case 1.

CASE 3 . The index $i^{1}$ corresponds to an inequality in (9)-(10) and the index $i^{2}$ to an inequality in (13)-(14). The proof is straightforward when using the techniques of Lemma A.2.

CASE 4 . The indices $i^{1}, i^{2}$ both correspond to inequalities in (11)-(12). The proof is similar to the one of Lemma A.2, Case 2, unless the equations added are $\theta_{j^{1}}^{h^{1}}=\theta_{j^{2}}^{h^{2}}=0$ and $h^{1}=h^{2}$. The proof is then similar to the one used in Case 2 in this proof.

CASE 5. The index $i^{1}$ corresponds to an inequality in (11)-(12) and the index $i^{2}$ to an inequality in (13)-(14). The proof is straightforward when using the techniques of Lemma A.2.

CASE 6 . The indices $i^{1}, i^{2}$ both correspond to inequalities in (13)-(14). In this case $0=\bar{t}=1$, a contradiction, so no solution to $\psi_{i^{1}, i^{2}}^{r}(\bar{\xi})$ exists, and transversality of $\psi_{i^{1}, i^{2}}^{r}$ holds trivially.

The lemma now follows from the parametric transversality theorem.

Q.E.D.

Before we continue with the second regular constraint system that describes $\widetilde{P}(r)$ in a neighborhood of $t=1$ when $J^{0}(r) \cup J_{-}^{1}(r) \neq \emptyset$, we derive some further properties of $\widetilde{P}(r)$ for $r$ with $J^{0}(r) \cup J_{-}^{1}(r) \neq \emptyset$. These properties are needed to show that the second system of equations is a regular constraint system. First, when $J^{0}(r) \neq \emptyset$, we show that generically it cannot be the case that for a solution in $\widetilde{P}(r)$ with $\bar{t}=1$, there is an asset $j \in J^{0}(r)$ with two shadow prices $\lambda_{j}^{h^{1},-}=\lambda_{j}^{h^{2},-}=0$. By definition of the homotopy one such shadow price must be zero. But, generically, two will never be simultaneously zero at $t=1$ when the asset market $j$ is closed. Second, when $J_{-}^{1}(r) \neq \emptyset$, we show that generically it cannot be the case that for a solution in $\widetilde{P}(r)$ with $\bar{t}=1$, there is an asset $j \in J_{-}^{1}(r)$ with $\theta_{j}^{h^{1}}=0$ and $\lambda_{j}^{h^{2},-}=0$, where $h^{1}$ is the only household such that $r_{j}^{h^{1}}=-1$ and $h^{2} \neq h^{1}$. Intuitively, as with $h^{1}$ the last agent leaves asset market $j$ at $t=1$, there is generically no other agent $h^{2}$ for whom the shadow price $\lambda_{j}^{h^{2},-}$ hits zero exactly at that point.

Fix some $r \in R$ with $J^{0}(r) \cup J_{-}^{1}(r) \neq \emptyset$. Define subsets $H_{j}^{-}(r), H_{j}^{0}(r)$ and $H_{j}^{+}(r)$ of $H$ as follows,

$$
\begin{aligned}
H_{j}^{-}(r) & =\left\{h \in H \mid r_{j}^{h}=-1\right\}, \\
H_{j}^{0}(r) & =\left\{h \in H \mid r_{j}^{h}=0\right\}, \\
H_{j}^{+}(r) & =\left\{h \in H \mid r_{j}^{h}=+1\right\} .
\end{aligned}
$$

For $j \in J^{0}(r) \cup J_{-}^{1}(r)$, fix subsets $\widetilde{H}_{j}^{0}(r)$ of $H_{j}^{0}(r)$, where $\widetilde{H}_{j}^{0}(r) \neq \emptyset$ if $j \in J^{0}(r)$. Moreover, to show the desired properties of $\widetilde{P}(r)$ we only need to consider cases where at least one of the subsets $\widetilde{H}_{j}^{0}(r), j \in J^{0}(r)$, contains at least two elements, or at least one of the subsets $\widetilde{H}_{j}^{0}(r), j \in J_{-}^{1}(r)$, is non-empty. The non-empty set of assets satisfying one of these two requirements is denoted $\widetilde{J}$. Fix an asset $\widetilde{j} \in \widetilde{J}$. A solution $\left(\bar{t}, \bar{q}, \bar{\theta}^{-}, \bar{\theta}^{+}, \bar{\theta}, \bar{c}\right) \in \widetilde{P}(r)$ that satisfies $\bar{t}=1$ and, for $j \in J^{0}(r) \cup J_{-}^{1}(r)$, $\bar{\lambda}_{j}^{h,-}=0$ if and only if $h \in \widetilde{H}_{j}^{0}(r)$, has to be a solution to the following system of equations and 
inequalities, even though the reverse is not necessarily true,

$$
\begin{aligned}
& \lambda_{j}^{h,-}=0, \quad(h, j) \in R^{-}(r), \\
& \lambda_{j}^{h,+}=0, \quad(h, j) \in R^{+}(r), \\
& \theta_{j}^{h}=0, \quad(h, j) \in R^{0}(r), \\
& c_{0}^{h}-e_{0}^{h}+\sum_{j \in J_{h}^{-}(r)} \theta_{j}^{h}\left(q_{j}-k_{j}\right)+\sum_{j \in J_{h}^{+}(r)} \theta_{j}^{h}\left(q_{j}+k_{j}\right)=0, \quad h \in H, \\
& c_{s}^{h}-e_{s}^{h}-\sum_{j \in J_{h}^{-}(r) \cup J_{h}^{+}(r)} \theta_{j}^{h} d_{s}^{j}=0, \quad h \in H, s \in S, \\
& \lambda_{j}^{h,-}+\partial_{c_{0}^{h}} u^{h}\left(c^{h}\right)\left(q_{j}-k_{j}\right)-\sum_{s=1}^{S} \partial_{c_{s}^{h}} u^{h}\left(c^{h}\right) d_{s}^{j}=0, \quad h \in H, j \in J, \\
& \lambda_{j}^{h,+}-\partial_{c_{0}^{h}} u^{h}\left(c^{h}\right)\left(q_{j}+k_{j}\right)+\sum_{s=1}^{S} \partial_{c_{s}^{h}} u^{h}\left(c^{h}\right) d_{s}^{j}=0, \quad h \in H, j \in J, \\
& t \sum_{h \in H} \theta_{j}^{h}+(1-t)\left(q_{j}^{0}-q_{j}\right)+t \frac{\varphi\left(\prod_{h=1}^{H} \lambda_{j}^{h,-}\right)}{1+e^{q_{j}}}=0, \quad j \in J \backslash \widetilde{J}, \\
& \lambda_{j}^{h(j),-}=0, \quad j \in \widetilde{J} \\
& \lambda_{\widetilde{j}}^{\widetilde{h},-}=0, \quad \text { if } \widetilde{j} \in J^{0}(r), \\
& \theta_{\widetilde{j}}^{\widetilde{h},-}=0, \quad \text { if } \widetilde{j} \in J_{-}^{1}(r), \\
& 1-t=0 \text {, } \\
& \lambda_{j}^{h,-}>0, \quad j \in J^{0}(r) \backslash \widetilde{J}, h \in H \backslash \widetilde{H}_{j}^{0}(r),
\end{aligned}
$$

where, for $j \in \widetilde{J}, h(j)$ is a uniquely determined choice in $\widetilde{H}_{j}^{0}(r), \widetilde{h} \in \widetilde{H}_{\tilde{j}}^{0}(r) \backslash\{h(\widetilde{j})\}$ if $\widetilde{j} \in J^{0}(r)$, and $\widetilde{h}$ is the uniquely determined household for which $r_{\tilde{j}}^{h}=-1$ if $\widetilde{j} \in J_{-}^{1}(r)$. In the system (27)-(39), the market clearing equations for assets in $\widetilde{J}$ have been omitted. Instead, for all such assets the shadow price $\lambda_{j}^{h(j),-}$ is required to be zero. Moreover, for one such asset, asset $\widetilde{j}$, one more constraint is specified. If the system can be shown to be regular, then there are evidently no solutions to it, as it is overdetermined.

For $r \in R$ with $J^{0}(r) \cup J_{-}^{1}(r) \neq \emptyset$, and a specification of sets $\widetilde{H}=\left(\widetilde{H}_{j}^{0}(r)\right)_{j \in J^{0}(r) \cup J_{-}^{1}(r)}$ such that $\widetilde{J} \neq \emptyset$, we define the open set $O^{r, \widetilde{H}}$ as the set of all elements $\left(t, q, \lambda^{-}, \lambda^{+}, \theta, c, e\right) \in(0, \infty) \times$ $Q \times \mathbb{R}^{3 H J} \times \mathbb{R}_{++}^{2 H(S+1)}$ satisfying (39). We define the function $\psi^{r, \widetilde{H}}: O^{r, \widetilde{H}} \rightarrow \mathbb{R}^{H(S+1)+3 H J+J+2}$ by associating the left-hand side of equations $(27)-(38)$ to $\psi^{r, \widetilde{H}}\left(t, q, \lambda^{-}, \lambda^{+}, \theta, c, e\right)$. For $e \in E$, the function $\psi^{r, \widetilde{H}, e}$ is defined by $\psi^{r, \widetilde{H}, e}(\cdot)=\psi^{r, \widetilde{H}}(\cdot, e)$.

Lemma A.4: There is a subset $E^{4}$ of $E$ of full Lebesgue measure such that for all $e \in E^{4}, r \in R$ with $J^{0}(r) \cup J_{-}^{1}(r) \neq \emptyset$, and $\widetilde{H}=\left(\widetilde{H}_{j}^{0}(r)\right)_{j \in J^{0}(r) \cup J_{-}^{1}(r)}$ with $\widetilde{J} \neq \emptyset, \psi^{r, \widetilde{H}, e}$ 历 $\{0\}$.

Proof: Fix some $r \in R$ and $\widetilde{H}$ satisfying the requirements of the lemma. We start out by showing that $\psi^{r, \widetilde{H}} \pitchfork\{0\}$. Let $\bar{\xi}$ be such that $\psi^{r, \widetilde{H}}(\bar{\xi})=0$. It has to be shown that the rows of the 
matrix $\bar{M}$ of partial derivatives of $\psi^{r, \widetilde{H}}$, evaluated at $\bar{\xi}$, are independent. Let $y \in \mathbb{R}^{H(S+1)+3 H J+J+1}$ satisfy $y^{\top} \bar{M}=0$. We index $y$ as before, let $y_{9, j}$ for $j \in \widetilde{J}$ be the component of $y$ corresponding to equalities (35), $y_{10}$ the component of $y$ corresponding to equalities (36) or (37), and $y_{11}$ the component of $y$ corresponding to equality (38).

It follows as in the proof of Lemma A.1 that $y_{4}=\cdots=y_{7}=0$. Consider some $j^{\prime} \in J \backslash \widetilde{J}$. If there is $h$ such that $r_{j^{\prime}}^{h} \neq 0$, then $0=y^{\top} \partial_{\theta_{j^{\prime}}} \psi^{r, \widetilde{H}}(\bar{\xi})=y_{8, j^{\prime}}$. Otherwise, for all $h \in H, r_{j^{\prime}}^{h}=0$. Since $j^{\prime} \notin \widetilde{J}$, there is a uniquely determined $h^{\prime}$ such that $\bar{\lambda}_{j^{\prime}}^{h^{\prime},-}=0$, and by $(39), \bar{\lambda}_{j^{\prime}}^{h,-}>0$ for $h \neq h^{\prime}$. Now, $0=y^{\top} \partial_{\lambda_{j^{\prime}}^{h^{\prime},-}} \psi^{r, \widetilde{H}}(\bar{\xi})=\left[\varphi^{\prime}\left(\prod_{h=1}^{H} \bar{\lambda}_{j^{\prime}}^{h,-}\right) \prod_{h \neq h^{\prime}} \bar{\lambda}_{j^{\prime}}^{h,-} /\left(1+e^{\left.\bar{q}_{j^{\prime}}\right)}\right)\right] y_{8, j^{\prime}}$ implies $y_{8, j^{\prime}}=0$. We have shown that $y_{8} \stackrel{j^{\prime}}{=} 0$. The remainder of the proof is straightforward.

We conclude that $\psi^{r, \widetilde{H}} \pitchfork\{0\}$. The lemma then follows directly from the parametric transversality theorem.

Q.E.D.

We are now in the position to formulate a second system of equalities and inequalities that applies for $r$ with $J^{0}(r) \cup J_{-}^{1}(r) \neq \emptyset$ in a neighborhood of $t=1$. Let $\pi$ be a function from $J^{0}(r)$ into $\{1, \ldots, H\}$. When $\pi(j)=h^{\prime}$ it is required that $\lambda_{j}^{h^{\prime},-}$ is minimal over all households, so household $h^{\prime}$. By Lemma A.4 it follows that for a generic economy, for solutions in a neighborhood of $t=1$, $\lambda_{j}^{h,-}>0$ if $h \neq h^{\prime}$. Similarly, for $j \in J_{-}^{1}(r)$, by Lemma A.4, it holds for a generic economy that $\lambda_{j}^{h,-}>0$ in a neighborhood of $t=1$ if $r_{j}^{h}=0$.

Consider any sign vector $r \in R$ and any function $\pi: J^{0}(r) \rightarrow\{1, \ldots, H\}$. If $\left(\bar{t}, \bar{q}, \bar{\lambda}^{-}, \bar{\lambda}^{+}, \bar{\theta}, \bar{c}\right) \in$ $\widetilde{P}(r), \bar{t}=1$, and $\pi(j)$, for $j \in J^{0}(r)$, is the (generically unique) household for which $\bar{\lambda}_{j}^{h,-}$ equals zero, then the following system of equalities and inequalities coincides with $\widetilde{P}(r)$ in a neighborhood 
of $\left(\bar{t}, \bar{q}, \bar{\lambda}^{-}, \bar{\lambda}^{+}, \bar{\theta}, \bar{c}\right)$.

$$
\begin{aligned}
\lambda_{j}^{h,-}=0, & \quad(h, j) \in R^{-}(r), \\
\lambda_{j}^{h,+}=0, & (h, j) \in R^{+}(r), \\
\theta_{j}^{h}=0, & (h, j) \in R^{0}(r), \\
c_{0}^{h}-e_{0}^{h}+\sum_{j \in J_{h}^{-}(r)} \theta_{j}^{h}\left(q_{j}-k_{j}\right)+\sum_{j \in J_{h}^{+}(r)} \theta_{j}^{h}\left(q_{j}+k_{j}\right)=0, \quad h \in H, & \\
c_{s}^{h}-e_{s}^{h}-\sum_{j \in J_{h}^{-}(r) \cup J_{h}^{+}(r)} \theta_{j}^{h} d_{s}^{j}=0, & h \in H, s \in S, \\
\lambda_{j}^{h,-}+\partial_{c_{0}^{h}} u^{h}(c)\left(q_{j}-k_{j}\right)-\sum_{s=1}^{S} \partial_{c_{s}^{h}} u^{h}(c) d_{s}^{j}=0, & h \in H, j \in J, \\
\lambda_{j}^{h,+}-\partial_{c_{0}^{h}} u^{h}(c)\left(q_{j}+k_{j}\right)+\sum_{s=1}^{S} \partial_{c_{s}^{h}} u^{h}(c) d_{s}^{j}=0, & h \in H, j \in J, \\
t \sum_{h \in H} \theta_{j}^{h}+(1-t)\left(q_{j}^{0}-q_{j}\right)+t \frac{\varphi\left(\prod_{h=1}^{H} \lambda_{j}^{h,-}\right)}{1+e^{q_{j}}=0,} \quad & j \in J, \\
\lambda_{j}^{h,-} \geq 0, & (h, j) \in R^{0}(r), j \notin J^{0}(r) \cup J_{-}^{1}(r), \\
\lambda_{j}^{h,+} \geq 0, & (h, j) \in R^{0}(r), \\
-\theta_{j}^{h} \geq 0, & (h, j) \in R^{-}(r), j \in J_{-}^{1}(r), \\
\theta_{j}^{h} \geq 0, & (h, j) \in R^{+}(r), \\
q_{j}-q_{j}^{0} \geq 0, & j \in J^{0}(r), \\
q_{j}^{0}-q_{j} \geq 0, & j \in J_{-}^{1}(r), \\
1-t \geq 0, & \\
\lambda_{j}^{h,-}>0, & j \in J^{0}(r), h \in H \backslash\{\pi(j)\}, \\
\lambda_{j}^{h,-}>0, & j \in J_{-}^{1}(r), h \in H_{j}^{0}(r) .
\end{aligned}
$$

Notice that the system of equalities remains the same for both regular constraint systems. This fact explains why we can use a single homotopy, even though we need two regular constraint systems for the proofs. It remains to be shown that the system above is a regular constraint system. The proof proceeds in three steps, as was the case for the first regular constraint system. The intuition behind the three steps remains the same. First, we prove that the set of zeros of the homotopy is 1-dimensional, next, we prove that boundaries are hit transversely, and finally we prove that never two boundaries are hit simultaneously.

For $r \in R$ with $J^{0}(r) \cup J_{-}^{1}(r) \neq \emptyset$ and $\pi: J^{0}(r) \rightarrow\{1, \ldots, H\}$, we define the open set $O^{r, \pi}$ as the set of all elements $\left(t, q, \lambda^{-}, \lambda^{+}, \theta, c, e\right) \in(0, \infty) \times Q \times \mathbb{R}^{3 H J} \times \mathbb{R}_{++}^{2 H(S+1)}$ that satisfy the inequalities (48)-(56) with strict inequality. We define a function $\psi^{r, \pi}: O^{r, \pi} \rightarrow \mathbb{R}^{H(S+1)+3 H J+J}$, by associating the left-hand side of equations (40)-(47) to $\psi^{r, \pi}\left(t, q, \lambda^{-}, \lambda^{+}, \theta, c, e\right)$. For $e \in E$, the function $\psi^{r, \pi, e}$ is defined by $\psi^{r, \pi, e}(\cdot)=\psi^{r, \pi}(\cdot, e)$. 
Lemma A.5: There is a subset $E^{5}$ of $E$ of full Lebesgue measure such that for all $e \in E^{5}, r \in R$ with $J^{0}(r) \cup J_{-}^{1}(r) \neq \emptyset$, and $\pi: J^{0}(r) \rightarrow\{1, \ldots, H\}, \psi^{r, \pi, e}$ 历 $\{0\}$.

Proof: The proof is parallel to the one of Lemma A.1. Q.E.D.

For the final proof that $\widetilde{P}(r)$ is a compact 1-dimensional manifold, we only need to consider the case where inequality (54) gets binding. The reason is that the system of equalities and inequalities (40)-(56) is only needed in a neighborhood of $t=1$, and Lemma A.7 will show that it cannot happen that two inequalities get binding simultaneously. We denote the index corresponding to inequality (54) by $\tau$.

For $r \in R$ with $J^{0}(r) \cup J_{-}^{1}(r) \neq \emptyset$ and $\pi: J^{0}(r) \rightarrow\{1, \ldots, H\}$, we define the open set $O_{\tau}^{r, \pi}$ as the set of all elements $\left(t, q, \lambda^{-}, \lambda^{+}, \theta, c, e\right) \in(0, \infty) \times Q \times \mathbb{R}^{3 H J} \times \mathbb{R}_{++}^{2 H(S+1)}$ that satisfy the inequalities (48)-(53), (55)-(56) with strict inequality. We define a function $\psi_{\tau}^{r, \pi}: O_{\tau}^{r, \pi} \rightarrow \mathbb{R}^{H(S+1)+3 H J+J+1}$, by associating the left-hand side of equations (40)-(47) to $\psi_{\tau}^{r, \pi}\left(t, q, \lambda^{-}, \lambda^{+}, \theta, c, e\right)$, and the left-hand side of inequality (54). For $e \in E$, the function $\psi_{\tau}^{r, \pi, e}$ is defined by $\psi_{\tau}^{r, \pi, e}(\cdot)=\psi_{\tau}^{r, \pi}(\cdot, e)$.

Lemma A.6: There is a subset $E^{6}$ of $E$ of full Lebesgue measure such that for all $e \in E^{6}, r \in R$ with $J^{0}(r) \cup J_{-}^{1}(r) \neq \emptyset$, and $\pi: J^{0}(r) \rightarrow\{1, \ldots, H\}, \psi_{\tau}^{r, \pi, e}$ 历 $\{0\}$.

Proof: Fix some $r \in R$ and some $\pi: J^{0}(r) \rightarrow\{1, \ldots, H\}$. Then the proof is parallel to the one of Lemma A.2.

Q.E.D.

For $r \in R$ with $J^{0}(r) \cup J_{-}^{1}(r) \neq \emptyset$ and $\pi: J^{0}(r) \rightarrow\{1, \ldots, H\}$, we define the open set $\widetilde{O}^{r, \pi}$ as the set of all elements $\left(t, q, \lambda^{-}, \lambda^{+}, \theta, c, e\right) \in(0, \infty) \times Q \times \mathbb{R}^{3 H J} \times \mathbb{R}_{++}^{2 H(S+1)}$ that satisfy the inequalities (55)-(56) with strict inequality. The set of indices corresponding to (48)-(53) is denoted by $I$. For $i \in I$, we define a function $\widetilde{\psi}_{i}^{r, \pi}: \widetilde{O}^{r, \pi} \rightarrow \mathbb{R}^{H(S+1)+3 H J+J+2}$ by associating the left-hand side of equations (40)-(47) to $\widetilde{\psi}_{i}^{r, \pi}\left(t, q, \lambda^{-}, \lambda^{+}, \theta, c, e\right)$, the left-hand side of one inequality, indexed by $i$, in (48)-(53), and inequality (54). For $e \in E$, the function $\psi_{i}^{r, \pi, e}$ is defined by $\psi_{i}^{r, \pi, e}(\cdot)=\psi_{i}^{r, \pi}(\cdot, e)$.

LEMma A.7: There is a subset $E^{7}$ of $E$ of full Lebesgue measure such that for all $e \in E^{7}, r \in R$ with $J^{0}(r) \cup J_{-}^{1}(r) \neq \emptyset, \pi: J^{0}(r) \rightarrow\{1, \ldots, H\}$ and $i \in I, \psi_{i}^{r, \pi, e} \pitchfork\{0\}$.

Proof: Fix some $r \in R$, some $\pi: J^{0}(r) \rightarrow\{1, \ldots, H\}$, and some $i \in I$. We start out by showing that $\psi_{i}^{r, \pi} 历\{0\}$. Let $\bar{\xi}$ be such that $\psi_{i}^{r, \pi}(\bar{\xi})=0$. It has to be shown that the rows of the matrix $\bar{M}$ of partial derivatives of $\psi_{i}^{r, \pi}$, evaluated at $\bar{\xi}$, are independent. Let $y \in \mathbb{R}^{(H+1)(S+1)+H S+2}$ satisfy $y^{\top} \bar{M}=0$. We index $y$ as before, let $y_{9}$ be the component of $y$ corresponding to inequality $\tau$, and $y_{10}$ be the component of $y$ corresponding to inequality $i$. Three cases have to be distinguished, depending on which inequality $i$ has been chosen.

CASE 1. The index $i$ corresponds to an inequality in (48)-(49). As in the proof of Lemma A.2 it can be shown that $y_{4}=\cdots=y_{7}=0$. Fix an asset $j$. If $j \notin J^{0}(r)$, then there is $h^{\prime}$ such that $r_{j}^{h^{\prime}} \neq 0$, 
and $0=y^{\top} \partial_{\theta_{j}^{h^{\prime}}}(\bar{\xi})=y_{8, j}$. If $j \in J^{0}(r)$, then using that the equality $\lambda_{j}^{h,-}=0$ is not added for $j \in J^{0}(r)$, it follows that $0=y^{\top} \partial_{\lambda_{j}^{\pi(j),-}}(\bar{\xi})=\left[\varphi^{\prime}(0) \prod_{h \neq \pi(j)} \bar{\lambda}_{j}^{h,-} /\left(1+e^{\bar{q}_{j}}\right)\right] y_{8, j}$, and since $\bar{\lambda}_{j}^{h,-} \neq 0$ for $h \neq \pi(j)$, it follows that $y_{8, j}=0$. The remainder of the proof is standard.

CAse 2. The index $i$ corresponds to an inequality in (50)-(51). As in the proof of Lemma A.2 it can be shown that $y_{4}=\cdots=y_{7}=0$. Because of Case 1 there is no loss of generality in assuming that $\bar{\lambda}_{j}^{h,-}>0$ for $(h, j) \in R^{0}(r), j \notin J^{0}(r) \cup J_{-}^{1}(r)$, and $\lambda_{j}^{h,+}>0$ for $(h, j) \in R^{0}(r)$. Fix an asset $j$ and suppose the index $i$ corresponds to $\theta_{j^{\prime}}^{h^{\prime}}=0$. The derivative with respect to $\theta_{j}^{h}$ such that $r_{j}^{h} \neq 0$ can be used to show that $y_{8, j}=0$, unless $j \in J^{0}(r)$ or $j=j^{\prime}$ and there is no household $h$ but $h^{\prime}$ such that $r_{j^{\prime}}^{h} \neq 0$. In the former case it holds that $0=y^{\top} \partial_{\lambda_{j}^{\pi(j),-}}(\bar{\xi})=\left[\varphi^{\prime}(0) \prod_{h \neq \pi(j)} \bar{\lambda}_{j}^{h,-} /\left(1+e^{\bar{q}_{j}}\right)\right] y_{8, j}$, so $y_{8, j}=0$. In the latter case, $0=y^{\top} \partial_{\lambda_{j}^{h^{\prime},-}}(\bar{\xi})=\left[\varphi^{\prime}(0) \prod_{h \neq h^{\prime}} \bar{\lambda}_{j}^{h,-} /\left(1+e^{\bar{q}_{j}}\right)\right] y_{8, j}$, so $y_{8, j}=0$. The remainder of the proof is standard.

Case 3. The index $i$ corresponds to an inequality in (52)-(53). This case follows immediately by using the arguments of Case 1 .

We conclude that $\widetilde{\psi}_{i}^{r, \pi} \pitchfork\{0\}$. The lemma then follows directly from the parametric transversality theorem.

Q.E.D.

\section{A4: Proofs of the Main Theorems}

In this section the full power of the previous seven lemmas becomes apparent. Using these lemmas, the theory of regular constraint sets, and the theory of manifolds with generalized boundary we can now prove our main results.

\section{Proof of TheOREM 4.2:}

Consider the set $E^{*}=E^{1} \cap E^{2} \cap E^{3} \cap E^{4} \cap E^{5} \cap E^{6} \cap E^{7}$. This set has full Lebesgue measure by Lemmas A.1 - A.7. Fix any $e \in E^{*}$.

We show first that $\widetilde{P}(r)$ is a 1-dimensional $C^{2}$ manifold with boundary. Consider any point $\left(\bar{t}, \bar{q}, \bar{\lambda}^{-}, \bar{\lambda}^{+}, \bar{\theta}, \bar{c}\right)$ in $\widetilde{P}(r)$. It is sufficient to show that $\widetilde{P}(r) \cap N$ is a 1-dimensional $C^{2}$ manifold with boundary, where $N$ is an open neighborhood of $\left(\bar{t}, \bar{q}, \bar{\lambda}^{-}, \bar{\lambda}^{+}, \bar{\theta}, \bar{c}\right)$ such that $\bar{t}<1$ implies $t<1$ for all $\left(t, q, \lambda^{-}, \lambda^{+}, \theta, c\right) \in N$. Two cases have to be considered, Case 1 where either $J^{0}(r) \cup J_{-}^{1}(r)=\emptyset$ or $J^{0}(r) \cup J_{-}^{1}(r) \neq \emptyset$ and $\bar{t}<1$, and Case 2 where $J^{0}(r) \cup J_{-}^{1}(r) \neq \emptyset$ and $\bar{t}=1$.

CASE 1. We define the function $\widetilde{g}: N \rightarrow \mathbb{R}^{H(S+1)+3 H J+J}$ by associating the left-hand side of equations (1)-(8) to $\widetilde{g}\left(t, q, \lambda^{-}, \lambda^{+}, \theta, c\right)$, and the function $\widetilde{h}: N \rightarrow \mathbb{R}^{\left|R^{0}(r)\right|+H J+2}$ by associating the left-hand side of inequalities (9)-(14) to $\widetilde{h}\left(t, q, \lambda^{-}, \lambda^{+}, \theta, c\right)$. We show that $M[\widetilde{g}, \widetilde{h}]$ is a regular constraint set. Consider any $\left(t, q, \lambda^{-}, \lambda^{+}, \theta, c\right) \in M[\widetilde{g}, \widetilde{h}]$. It has to be shown that

$$
\left\{\partial \widetilde{g}_{i}\left(t, q, \lambda^{-}, \lambda^{+}, \theta, c\right), i \in I^{1}, \partial \widetilde{h}_{i}\left(t, q, \lambda^{-}, \lambda^{+}, \theta, c\right), i \in I^{0}\left(t, q, \lambda^{-}, \lambda^{+}, \theta, c\right)\right\}
$$

is a set of independent vectors. When $I^{0}\left(t, q, \lambda^{-}, \lambda^{+}, \theta, c\right)=\emptyset$, this is a consequence of Lemma A.1, when $I^{0}\left(t, q, \lambda^{-}, \lambda^{+}, \theta, c\right) \neq \emptyset$, a consequence of Lemma A.2. Suppose $\left|I^{0}\left(t, q, \lambda^{-}, \lambda^{+}, \theta, c\right)\right| \geq 2$ and choose two elements, say $i^{\prime}$ and $i^{\prime \prime}$ from $I^{0}\left(t, q, \lambda^{-}, \lambda^{+}, \theta, c\right)$. It follows from Lemma A.3 that

$$
\left\{\partial \widetilde{g}_{i}\left(t, q, \lambda^{-}, \lambda^{+}, \theta, c\right), i \in I^{1}, \partial \widetilde{h}_{i^{\prime}}\left(t, q, \lambda^{-}, \lambda^{+}, \theta, c\right), \partial \widetilde{h}_{i^{\prime \prime}}\left(t, q, \lambda^{-}, \lambda^{+}, \theta, c\right)\right\}
$$


is a set of independent vectors, which leads to a contradiction as the set contains $H(S+1)+$ $3 H J+J+2$ vectors of dimension $H(S+1)+3 H J+J+1$. Consequently, $\left|I^{0}\left(t, q, \lambda^{-}, \lambda^{+}, \theta, c\right)\right| \leq 1$. Moreover, $M[\widetilde{g}, \widetilde{h}]$ is a $C^{2}$ regular constraint set. Since $M[\widetilde{g}, \widetilde{h}]=P(r) \cap N$, it follows that $\widetilde{P}(r) \cap N$ is a 1-dimensional $C^{2}$ manifold with boundary, and that the boundary of $\widetilde{P}(r) \cap N$ is given by the elements $\left(t, q, \lambda^{-}, \lambda^{+}, \theta, c\right) \in M[\widetilde{g}, \widetilde{h}]$ for which $\left|I^{0}\left(t, q, \lambda^{-}, \lambda^{+}, \theta, c\right)\right|=1$. Therefore, a point in $\widetilde{P}(r) \cap N$ is a boundary point if and only if exactly one of the inequalities in (9)-(14) holds with equality.

CASE 2. Suppose that, for some $j \in J^{0}(r)$, there are at least two households such that $\bar{\lambda}_{j}^{h,-}=0$, or, for some $j \in J_{-}^{1}(r)$, there is at least one household $h \in H_{j}^{0}(r)$ such that $\bar{\lambda}_{j}^{h,-}=0$. For $j \in J^{0}(r) \cup J_{-}^{1}(r)$, define the set $\widetilde{H}_{j}^{0}(r)$ as the set of households in $H_{j}^{0}(r)$ for which $\bar{\lambda}_{j}^{h,-}=0$. Then $\left(\bar{t}, \bar{q}, \bar{\lambda}^{-}, \bar{\lambda}^{+}, \bar{\theta}, \bar{c}\right)$ is a solution to (27)-(39). It follows from Lemma A.4 that $\partial \psi^{r, \widetilde{H}, e} \pitchfork\{0\}$. Counting the number of equations and unknowns it follows that $\left(\psi^{r, \widetilde{H}, e}\right)^{-1}(\{0\})=\emptyset$, contradicting that $\left(\psi^{r, \widetilde{H}, e}\right)^{-1}\left(\bar{t}, \bar{q}, \bar{\lambda}^{-}, \bar{\lambda}^{+}, \bar{\theta}, \bar{c}\right)=0$. Consequently, for $j \in J^{0}(r)$, there is at most one household $h$ such that $\bar{\lambda}_{j}^{h,-}=0$, and, for $j \in J_{-}^{1}(r)$, there is no household $h \in H_{j}^{0}(r)$ such that $\bar{\lambda}_{j}^{h,-}=0$.

We define the function $\widetilde{g}: N \rightarrow \mathbb{R}^{H(S+1)+3 H J+J}$ by associating the left-hand side of equations (40)-(47) to $\widetilde{g}\left(t, q, \lambda^{-}, \lambda^{+}, \theta, c\right)$, and the function $\widetilde{h}: N \rightarrow \mathbb{R}^{\left|R^{0}(r)\right|+H J+2}$ by associating the lefthand side of inequalities (48)-(54) to $\widetilde{h}\left(t, q, \lambda^{-}, \lambda^{+}, \theta, c\right)$. Moreover, it is possible to take $N$ such that (55) and (56) are satisfied for all $\left(t, q, \lambda^{-}, \lambda^{+}, \theta, c\right) \in N$. We show that $M[\widetilde{g}, \widetilde{h}]$ is a regular constraint set. Consider any $\left(t, q, \lambda^{-}, \lambda^{+}, \theta, c\right) \in M[\widetilde{g}, \widetilde{h}]$. It has to be shown that

$$
\left\{\partial \widetilde{g}_{i}\left(t, q, \lambda^{-}, \lambda^{+}, \theta, c\right), i \in I^{1}, \partial \widetilde{h}_{i}\left(t, q, \lambda^{-}, \lambda^{+}, \theta, c\right), i \in I^{0}\left(t, q, \lambda^{-}, \lambda^{+}, \theta, c\right)\right\}
$$

is a set of independent vectors. When $I^{0}\left(t, q, \lambda^{-}, \lambda^{+}, \theta, c\right)=\emptyset$, this is a consequence of Lemma A.5, when $I^{0}\left(t, q, \lambda^{-}, \lambda^{+}, \theta, c\right) \neq \emptyset$, a consequence of Lemma A.6. Suppose $\left|I^{0}\left(t, q, \lambda^{-}, \lambda^{+}, \theta, c\right)\right| \geq 2$ and choose two elements, say $i^{\prime}$ and $i^{\prime \prime}$ from $I^{0}\left(t, q, \lambda^{-}, \lambda^{+}, \theta, c\right)$. It follows from Lemma A.7 that

$$
\left\{\partial \widetilde{g}_{i}\left(t, q, \lambda^{-}, \lambda^{+}, \theta, c\right), i \in I^{1}, \partial \widetilde{h}_{i^{\prime}}\left(t, q, \lambda^{-}, \lambda^{+}, \theta, c\right), \partial \widetilde{h}_{i^{\prime \prime}}\left(t, q, \lambda^{-}, \lambda^{+}, \theta, c\right)\right\}
$$

is a set of independent vectors, which leads to a contradiction as the set contains $H(S+1)+$ $3 H J+J+2$ vectors of dimension $H(S+1)+3 H J+J+1$. Consequently, $\left|I^{0}\left(t, q, \lambda^{-}, \lambda^{+}, \theta, c\right)\right| \leq 1$. Moreover, $M[\widetilde{g}, \widetilde{h}]$ is a $C^{2}$ regular constraint set. Since $M[\widetilde{g}, \widetilde{h}]=P(r) \cap N$, it follows that $\widetilde{P}(r) \cap N$ is a 1-dimensional $C^{2}$ manifold with boundary, and that the boundary of $\widetilde{P}(r) \cap N$ is given by the elements $\left(t, q, \lambda^{-}, \lambda^{+}, \theta, c\right) \in M[\widetilde{g}, \widetilde{h}]$ for which $\left|I^{0}\left(t, q, \lambda^{-}, \lambda^{+}, \theta, c\right)\right|=1$. Therefore, a point in $\widetilde{P}(r) \cap N$ is a boundary point if and only if exactly one of the inequalities in (48)-(54) holds with equality.

Next we show compactness of $\widetilde{P}(r)$, or, by Proposition 4.1, the equivalent result that $P(r)$ is compact. Let $\left(t^{n}, q^{n}\right)$ be a sequence in $P(r)$.

First we show that $\left(t^{n}, q^{n}\right)$ is bounded. Suppose not, then, without loss of generality, $\left\|q^{n}\right\| \rightarrow \infty$. Since $\left(t^{n}, q^{n}\right) \in P(r)$, it holds that $\widetilde{F}\left(t^{n}, q^{n}\right)=0$. Therefore,

$$
\begin{aligned}
0 & =\left(q^{0}-q^{n}\right) \cdot \widetilde{F}\left(t^{n}, q^{n}\right) \\
& =t^{n}\left(q^{0}-q^{n}\right) \cdot G\left(q^{n}\right)+t^{n} \sum_{j=1}^{J}\left(q_{j}^{0}-q_{j}^{n}\right) \frac{\varphi\left(\Lambda_{j}^{-}\left(q^{n}\right)\right)}{1+e^{q_{j}^{n}}}+\left(1-t^{n}\right)\left(q^{0}-q^{n}\right) \cdot\left(q^{0}-q^{n}\right) .
\end{aligned}
$$


We continue by analyzing the tree terms in (57). Notice that $G_{0}\left(q^{n}\right)+q^{n} G\left(q^{n}\right)$ is equal to minus the total transactions costs paid in the economy at prices $q^{n}$, and therefore less than or equal to zero. We find that

$$
\left(q^{0}-q^{n}\right) \cdot G\left(q^{n}\right)=q^{0} \cdot G\left(q^{n}\right)-q^{n} \cdot G\left(q^{n}\right) \geq q^{0} \cdot G\left(q^{n}\right)+G_{0}\left(q^{n}\right) \rightarrow \infty
$$

where the last part follows by Proposition 3.6. The first term in (57) equals $t^{n}$ times an expression that goes to infinity. The second term is bounded below if $q_{j}^{n} \rightarrow+\infty$ and tends to infinity if $q_{j}^{n} \rightarrow-\infty$. Since $\left\|q^{n}\right\| \rightarrow \infty$ implies that $\left(q^{0}-q^{n}\right) \cdot\left(q^{0}-q^{n}\right) \rightarrow \infty$, the third term equals $\left(1-t^{n}\right)$ times an expression that goes to infinity. It follows that the entire expression in (57) goes to infinity, a contradiction to the fact that it is equal to zero. Consequently, the sequence $\left(t^{n}, q^{n}\right)$ is bounded.

Next we show that $P(r)$ is closed. Consider a convergent sequence $\left(t^{n}, q^{n}\right)$ in $P(r)$, with limit $(\bar{t}, \bar{q})$. We prove first that $\bar{q} \in Q$. Suppose not, then $\bar{q} \in \partial Q$. We analyze again the expression in (57). As before, it follows by Proposition 3.6 that the first term equals $t^{n}$ times an expression going to infinity, the second term equals $t^{n}$ times an expression that is bounded, and since $q^{n}$ converges to a point in $\partial Q$, the third term equals $\left(1-t^{n}\right)$ times an expression that converges to a strictly positive number. We conclude that, for $n$ large, the expression in (57) is not equal to zero, giving rise to a contradiction. Consequently, $\bar{q} \in Q$. Since $\widetilde{F}$ is continuous on $[0,1] \times Q$, it follows that $\widetilde{F}(\bar{t}, \bar{q})=0$, so $(\bar{t}, \bar{q}) \in P(r)$.

We conclude that $\widetilde{P}(r)$ is a compact, 1-dimensional $C^{2}$ manifold with boundary. It follows from standard arguments that the set $E^{*}$ can be chosen such that the closure of $E \backslash E^{*}$ has Lebesgue measure zero, see Herings (1997).

Finally, we show that the sets $\widetilde{P}(r)$ are linked together by their boundary points as described in the theorem. Consider a point $\left(\bar{t}, \bar{q}, \bar{\lambda}^{-}, \bar{\lambda}^{+}, \bar{\theta}, \bar{c}\right)$ in the boundary of $\widetilde{P}(r)$.

Suppose $J^{0}(r) \cup J_{-}^{1}(r)=\emptyset$, or $J^{0}(r) \cup J_{-}^{1}(r) \neq \emptyset$ and $\bar{t}<1$. It holds that $\left(\bar{t}, \bar{q}, \bar{\lambda}^{-}, \bar{\lambda}^{+}, \bar{\theta}, \bar{c}\right)$ is a solution to (1)-(14) with exactly one of the inequalities (9)-(14) holding with equality.

If one of the inequalities in (9)-(12) holds with equality, then $\left(\bar{t}, \bar{q}, \bar{\lambda}^{-}, \bar{\lambda}^{+}, \bar{\theta}, \bar{c}\right)$ belongs to $(0,1) \times Q \times \mathbb{R}^{H J} \times \mathbb{R}^{H J} \times \mathbb{R}^{H J} \times \mathbb{R}^{H(1+S)}$. If (9) holds with equality for some $\left(h^{\prime}, j^{\prime}\right) \in R^{0}(r)$, then define $\widetilde{r}$ by $\widetilde{r}_{j^{\prime}}^{h^{\prime}}=-1$, and $\widetilde{r}_{j}^{h}=r_{j}^{h}$ for $(h, j) \neq\left(h^{\prime}, j^{\prime}\right)$. If (10) holds with equality for some $\left(h^{\prime}, j^{\prime}\right) \in R^{0}(r)$, then define $\widetilde{r}$ by $\widetilde{r}_{j^{\prime}}^{h^{\prime}}=+1$, and $\widetilde{r}_{j}^{h}=r_{j}^{h}$ for $(h, j) \neq\left(h^{\prime}, j^{\prime}\right)$. If (11) or (12) holds with equality for some $\left(h^{\prime}, j^{\prime}\right)$, then define $\widetilde{r}$ by $\widetilde{r}_{j^{\prime}}^{h^{\prime}}=0$, and $\widetilde{r}_{j}^{h}=r_{j}^{h}$ for $(h, j) \neq\left(h^{\prime}, j^{\prime}\right)$. Since either $J^{0}(\widetilde{r}) \cup J^{1}(\widetilde{r})=\emptyset$, or $J^{0}(\widetilde{r}) \cup J^{1}(\widetilde{r}) \neq \emptyset$ and $\bar{t}<1$, it is easily verified that $\left(\bar{t}, \bar{q}, \bar{\lambda}^{-}, \bar{\lambda}^{+}, \bar{\theta}, \bar{c}\right)$ is a solution to (1)-(14) when specified for $\widetilde{r}$, with exactly one of the inequalities (9)-(12) holding with equality. Therefore, $\left(\bar{t}, \bar{q}, \bar{\lambda}^{-}, \bar{\lambda}^{+}, \bar{\theta}, \bar{c}\right)$ is a boundary point of $\widetilde{P}(\widetilde{r})$. It is immediate that for any other choice for $\widetilde{r}$, the resulting system of equalities and inequalities does not admit $\left(\bar{t}, \bar{q}, \bar{\lambda}^{-}, \bar{\lambda}^{+}, \bar{\theta}, \bar{c}\right)$ as a solution.

If one of the inequalities in (13)-(14) holds with equality, then $\left(\bar{t}, \bar{q}, \bar{\lambda}^{-}, \bar{\lambda}^{+}, \bar{\theta}, \bar{c}\right)$ belongs to $\{0,1\} \times Q \times \mathbb{R}^{H J} \times \mathbb{R}^{H J} \times \mathbb{R}^{H J} \times \mathbb{R}^{H(1+S)}$. It is immediate that for any $\widetilde{r} \neq r$, the resulting system of equalities and inequalities (1)-(14) does not admit $\left(\bar{t}, \bar{q}, \bar{\lambda}^{-}, \bar{\lambda}^{+}, \bar{\theta}, \bar{c}\right)$ as a solution.

Suppose $J^{0}(r) \cup J_{-}^{1}(r) \neq \emptyset$ and $\bar{t}=1$. It holds that $\left(\bar{t}, \bar{q}, \bar{\lambda}^{-}, \bar{\lambda}^{+}, \bar{\theta}, \bar{c}\right)$ is a solution to (40)-(56) with exactly one of the inequalities (48)-(54) holding with equality. Since $\bar{t}=1$, inequality (54) 
holds with equality. It follows that $\left(\bar{t}, \bar{q}, \bar{\lambda}^{-}, \bar{\lambda}^{+}, \bar{\theta}, \bar{c}\right)$ belongs to $\{1\} \times Q \times \mathbb{R}^{H J} \times \mathbb{R}^{H J} \times \mathbb{R}^{H J} \times$ $\mathbb{R}^{H(1+S)}$. It is immediate that for any $\widetilde{r} \neq r$, the resulting system of equalities and inequalities (1)-(14) does not admit $\left(\bar{t}, \bar{q}, \bar{\lambda}^{-}, \bar{\lambda}^{+}, \bar{\theta}, \bar{c}\right)$ as a solution.

Q.E.D.

Proof of Theorem 3.7:

The properties of $P(r)$ follow immediately from those of $\widetilde{P}(r)$ since the two sets are diffeomorphic by Proposition 4.1 .

Q.E.D.

Proof of THEOREM 3.8:

Consider the set $E^{*}$ of Theorem 3.7. For all $e \in E^{*}$, for all $r \in R$, the set $P(r)$ consists of a finite number of paths and loops. Each path in $P(r)$ has two boundary points. If it has a boundary point in $\{0,1\} \times Q$, then the boundary point does not belong to any $P(\bar{r})$ for $\bar{r} \neq r$. It is then a boundary point of $P$. If a path has a boundary point in $(0,1) \times Q$, then it is a boundary point of exactly one $P(\bar{r})$ with $\bar{r} \neq r$. So it is a boundary point of a path in $P(\bar{r})$. This path has another boundary point, either in $\{0,1\} \times Q$ or in $(0,1) \times Q$. In the former case, we have found a boundary point of $P$. In the latter case, there is exactly one $\widetilde{r}$ such that the boundary point is also a boundary point of an arc in $P(\widetilde{r})$, etc.

Since the cardinality of the set $R$ is finite, and each $P(r)$ consists of finitely many paths and loops, it will either be the case that eventually a path is generated with a boundary point in $\{0,1\} \times Q$, or a path is generated that has been generated before. In the latter case, we have found a piecewise $C^{2}$ loop of $P$. In the former case, the finite chain of paths constitutes a piecewise $C^{2}$ path of $P$ with boundary points belonging to $\{0,1\} \times Q$. It follows that $P$ is a compact 1 dimensional piecewise $C^{2}$ manifold with boundary, where the boundary is given by the intersection of $P$ and $\{0,1\} \times Q$. Notice that the argument above is nothing but a nonlinear version of the door-in door-out principle of Lemke and Howson (1964).

It is easy to see that there is a unique boundary point in $\{0\} \times Q$, since $\widetilde{F}(0, q)=q^{0}-q$, which has $q=q^{0}$ as the unique solution in $\widetilde{F}^{-1}(\{0\})$.

Q.E.D. 


\section{References}

Aiyagari, S.R., and M. Gertler (1991), "Asset Returns with Transaction Costs and Uninsured Individual Risk," Journal of Monetary Economics, 27, 311-331.

Arrow, K.J., and F.H. Hahn (1999), "Notes on Sequence Economies, Transaction Costs and Uncertainty," Journal of Economic Theory, 86, 203-218.

Brown, D.J., P.M. DeMarzo, and B.C. Eaves (1996a), "Computing Equilibria When Asset Markets Are Incomplete," Econometrica, 64, 1-27.

Brown, D.J., P.M. DeMarzo, and B.C. Eaves (1996b), "Computing Zeros of Sections of Vector Bundles Using Homotopies and Relocalization," Mathematics of Operations Research, 21, 26-43.

Constantinides, G.M. (1986), "Capital Market Equilibrium with Transaction Costs," Journal of Political Economy, 94, 842-862.

Debreu, G. (1959), Theory of Value, Yale University Press, New Haven.

Debreu, G. (1970), "Economies with a Finite Set of Equilibria," Econometrica, 38, 387-392.

DeMarzo, P.M., and B.C. Eaves (1996), "Computing Equilibria of GEI by Relocalization on a Grassmann Manifold," Journal of Mathematical Economics, 26, 479-497.

Dubey, P., J. Geanakoplos, and M. Shubik (1997), Default and Efficiency in a General Equilibrium Model with Incomplete Markets, Working Paper, Presented at the European Workshop on General Equilibrium Theory, Louvain-la-Neuve, Belgium, 1-55.

Duffie, D., and M.O. Jackson (1989), "Optimal Innovation of Futures Contracts," Review of Financial Studies, 2, 275-296.

Eaves, B.C., and K. Schmedders (1999), "General Equilibrium Models and Homotopy Methods," Journal of Economic Dynamics and Control, 23, 1249-1279.

Elul, R. (1997), "Financial Innovation, Precautionary Saving and the Risk-Free Rate," Journal of Mathematical Economics, 27, 113-131.

Garcia, C.B. and W.I. Zangwill (1981), Pathways to Solutions, Fixed Points, and Equilibria, Prentice-Hall Series in Computational Mathematics, Prentice-Hall, Englewood Cliffs.

Geanakoplos, J.D. (1990), "An Introduction to General Equilibrium with Incomplete Asset Markets," Journal of Mathematical Economics, 19, 1-38.

Heaton, J., and D.J. Lucas (1996), "Evaluating the Effects of Incomplete Markets on Risk Sharing and Asset Pricing," Journal of Political Economy, 104, 443-487.

Hens, T. (1991), Structure of General Equilibrium Models with Incomplete Markets, Doctoral Dis- 
sertation, University of Bonn.

Herings, P.J.J. (1997), "A Globally and Universally Stable Price Adjustment Process," Journal of Mathematical Economics, 27, 163-193.

Herings, P.J.J., and R. Peeters (2000), "A Differentiable Homotopy to Compute Nash Equilibria of $n$-Person Games," forthcoming in Economic Theory.

Jongen, H.Th., P. Jonker, and F. Twilt (1983), Nonlinear Optimization in $\mathbb{R}^{n}$, I. Morse Theory, Chebyshev Approximation, Methoden und Verfahren der Mathematischen Physik, 29, Peter Lang, Frankfurt.

Laitenberger, M. (1996), "Existence of Financial Market Equilibria with Transaction Costs," Ricerche Economiche, 50, 69-77.

Lemke, C.E., and J.T. Howson, Jr. (1964), "Equilibrium Points of Bimatrix Games," SIAM Journal on Applied Mathematics, 12, 413-423.

Magill, M., and W. Shafer (1991), "Incomplete Markets," in W. Hildenbrand and H. Sonnenschein (eds.), Handbook of Mathematical Economics, Volume IV, North-Holland, Amsterdam, 1523-1614.

Mas-Colell, A. (1985), The Theory of General Economic Equilibrium, A Differentiable Approach, Cambridge University Press, Cambridge.

Préchac, C. (1996), " Existence of Equilibrium in Incomplete Markets with Intermediation Costs," Journal of Mathematical Economics, 25, 373-380.

Rockafellar, R.T. (1970), Convex Analysis, Princeton University Press, Princeton.

Schmedders, K. (1998), "Computing Equilibria in the General Equilibrium Model with Incomplete Asset Markets," Journal of Economic Dynamics and Control, 22, 1375-1401.

Schmedders, K. (1999), "A Homotopy Algorithm and an Index Theorem for the General Equilibrium Model with Incomplete Asset Markets," Journal of Mathematical Economics, 32, 225-241.

Watson, L.T., S.C. Billups, and A.P. Morgan (1987), "HOMPACK: A Suite of Codes for Globally Convergent Homotopy Algorithms," ACM Transactions on Mathematical Software, 13, 281-310.

Yamazaki, A. (1991), "Equilibrium in Economies with Incomplete Markets and Outside Money: Transaction Costs and Existence," Working Paper, 1-34. 


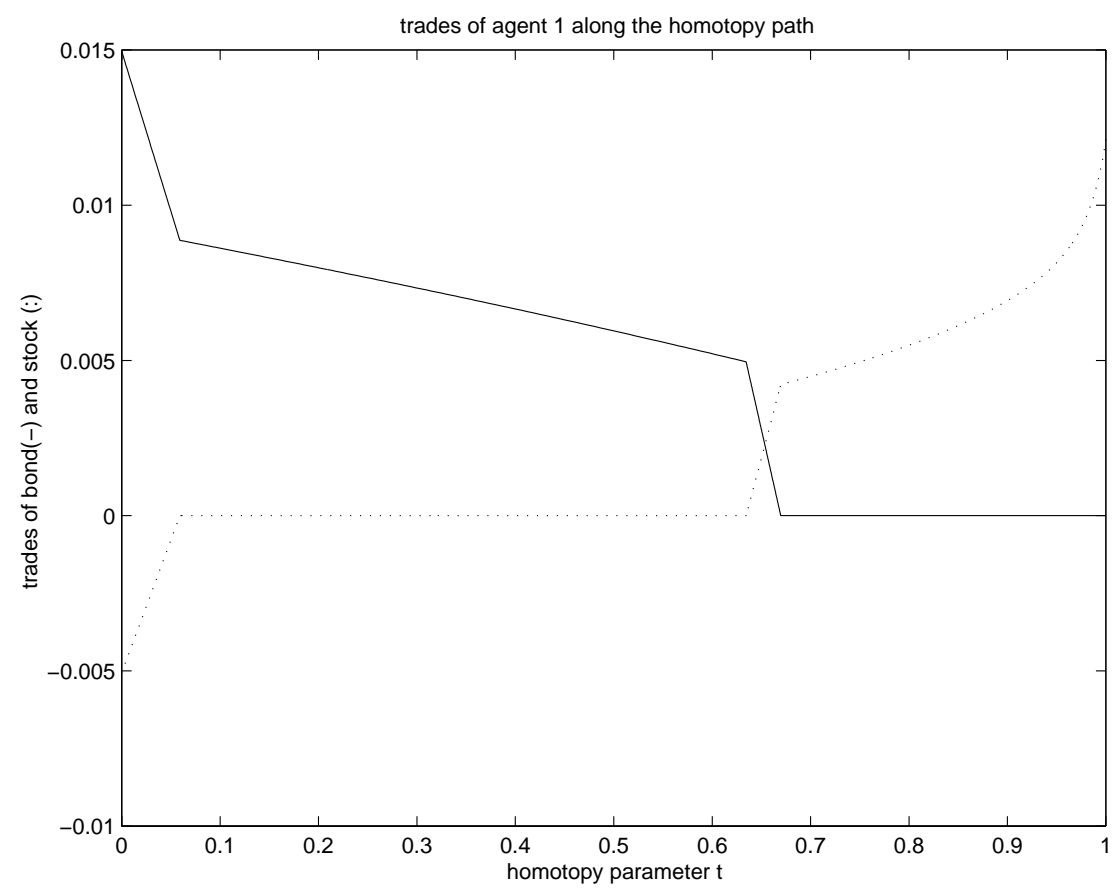

FigURE 2: Asset trades of agent 1 along the homotopy path. 


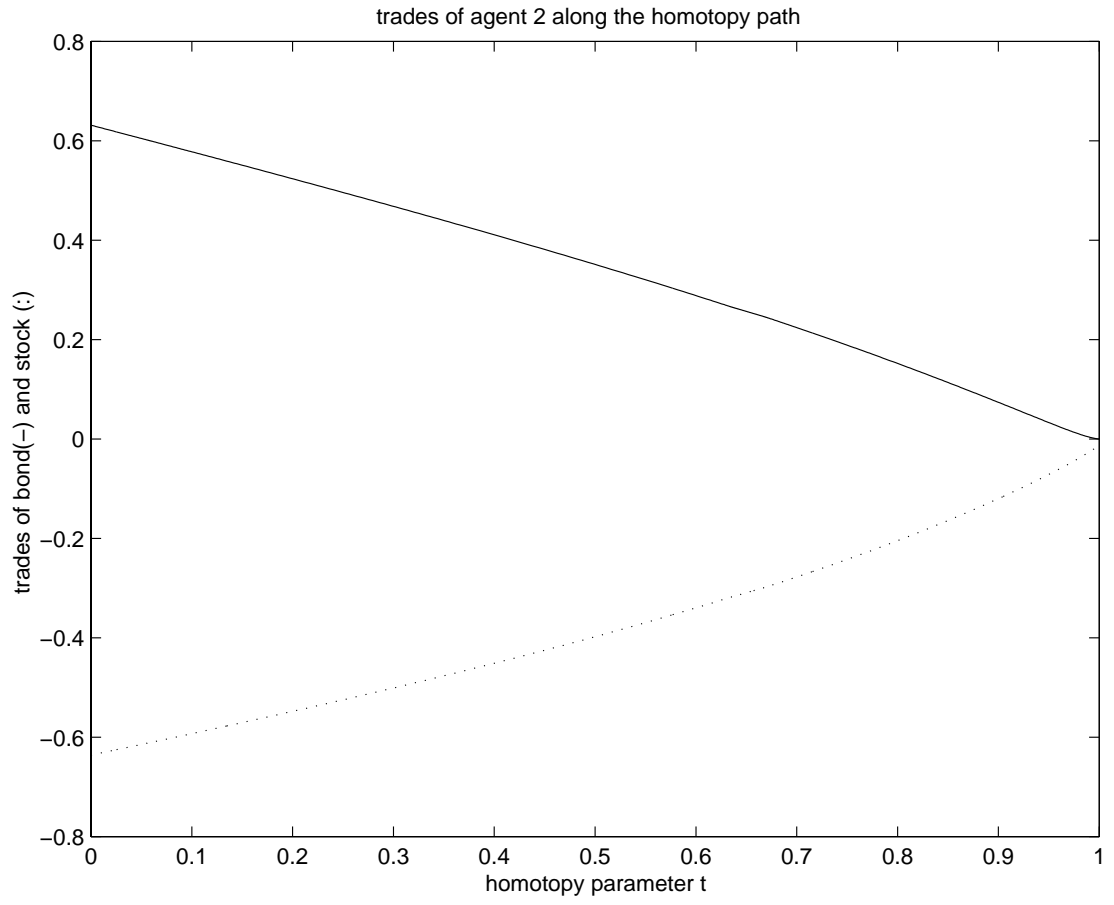

FiguRE 3: Asset trades of agent 2 along the homotopy path. 


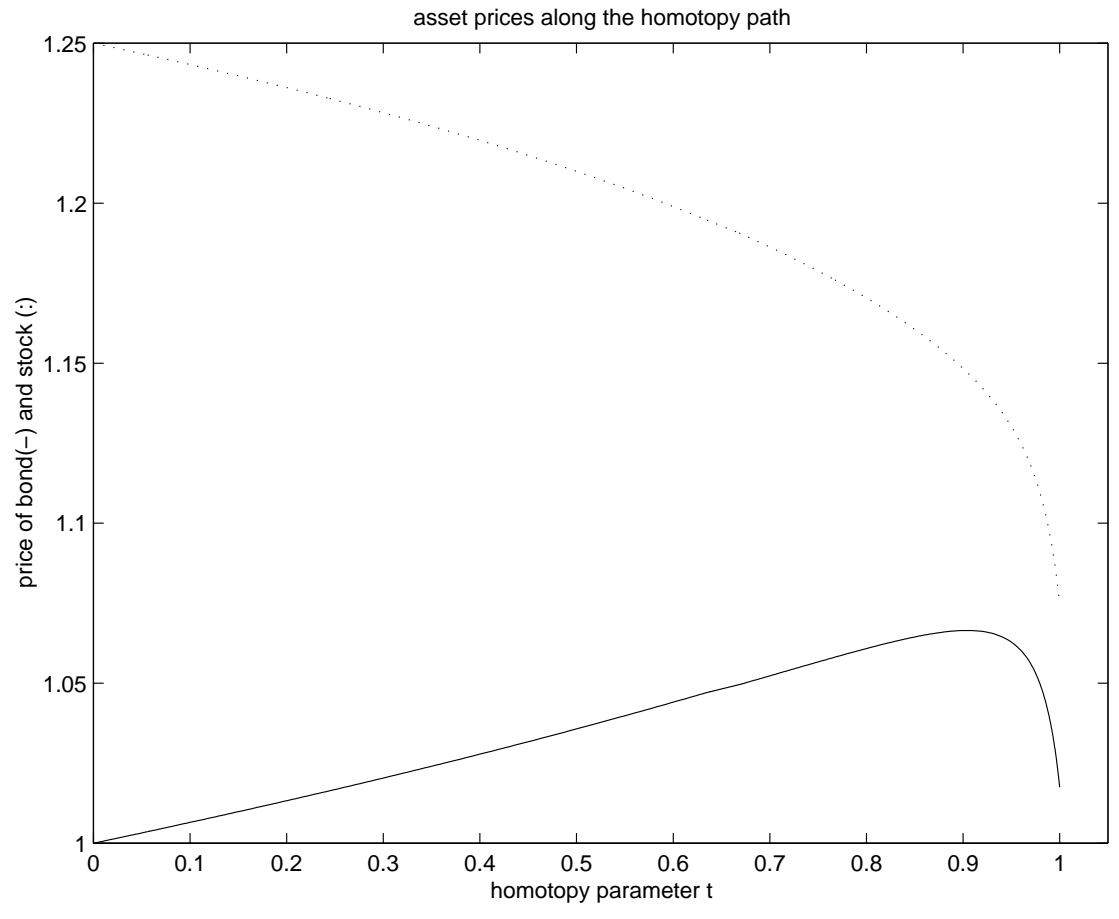

Figure 4: Asset prices along the homotopy path. 


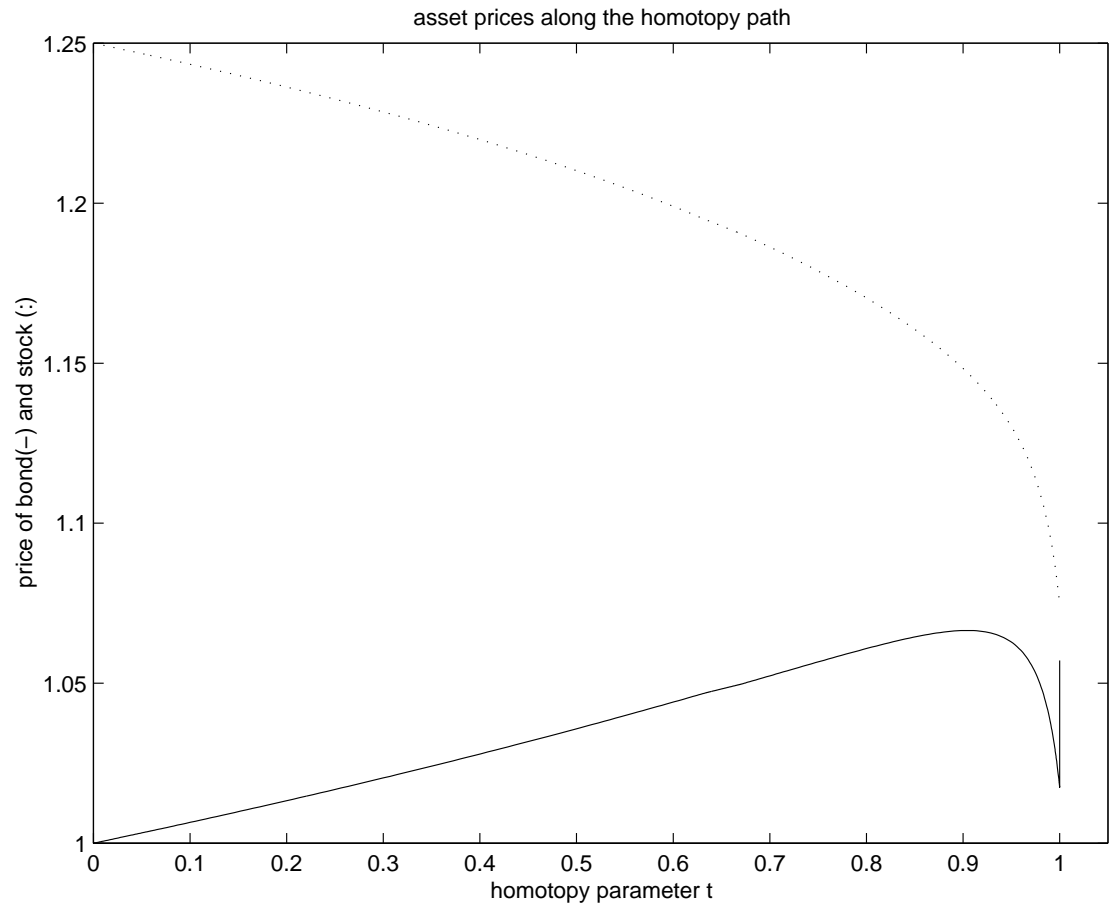

FIgURE 5: Continuum of equilibrium prices. 


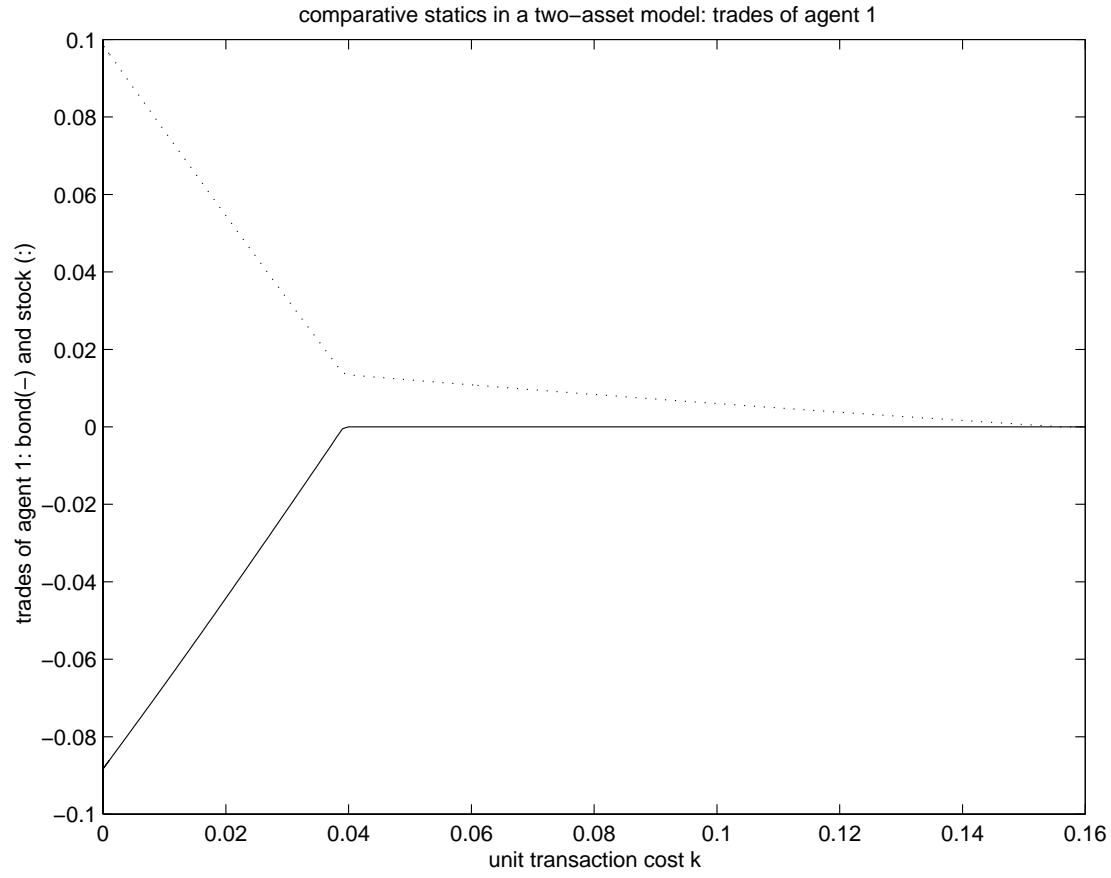

FiguRE 6: Comparative statics: equilibrium trades of agent 1. 


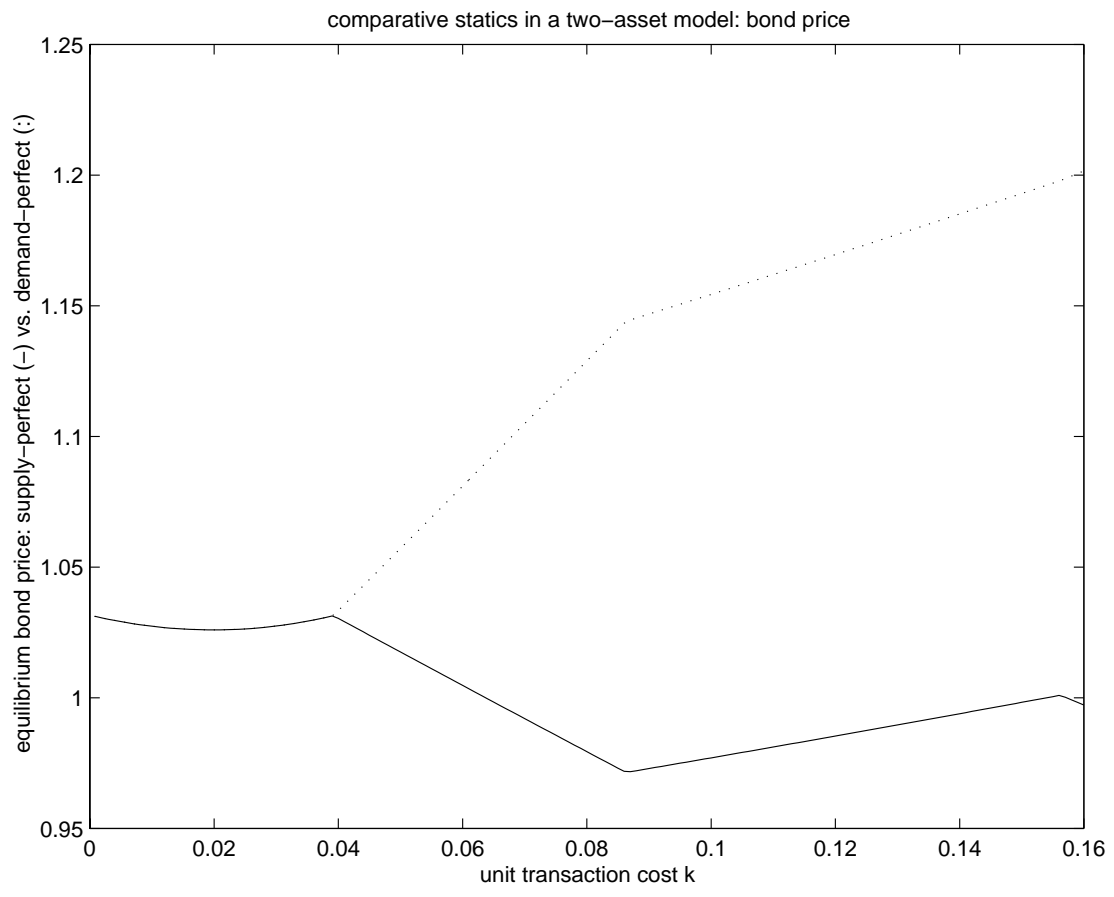

FIGURE 7: Comparative statics: equilibrium bond price. 


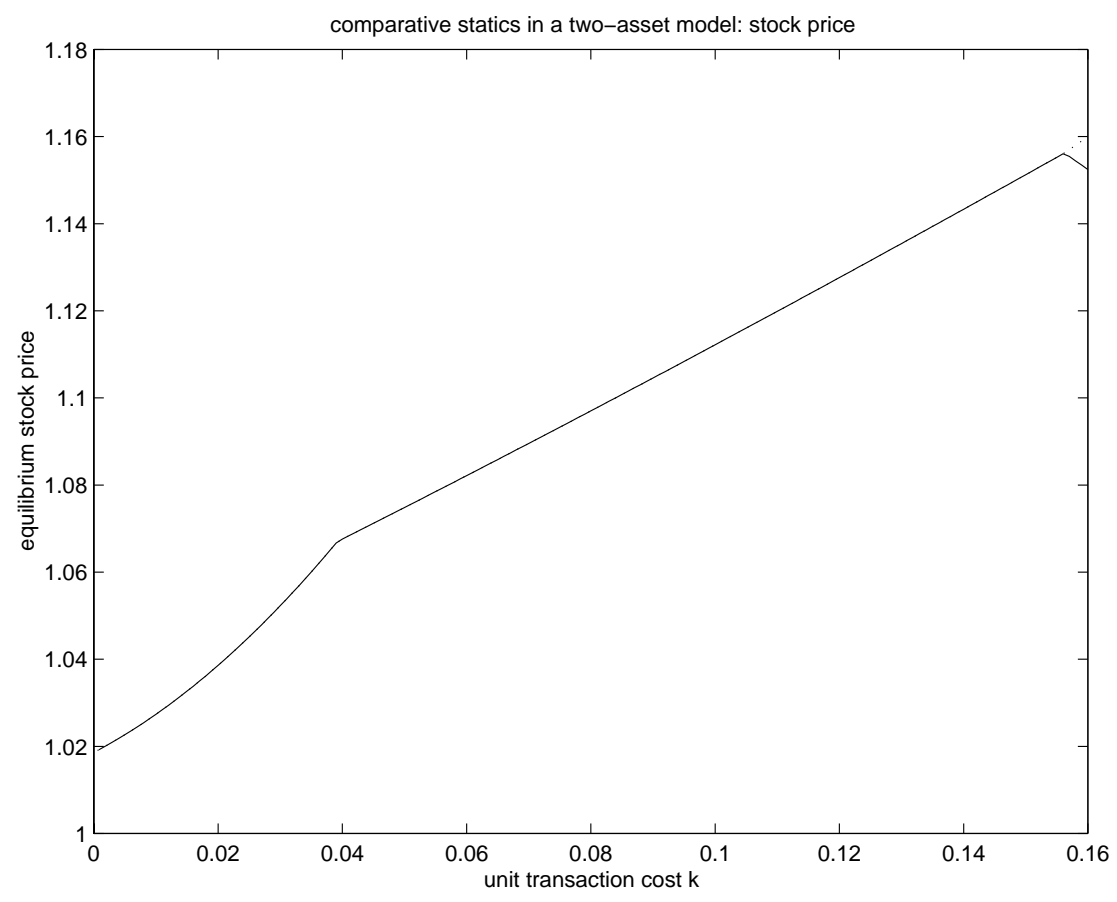

Figure 8: Comparative statics: equilibrium stock price. 


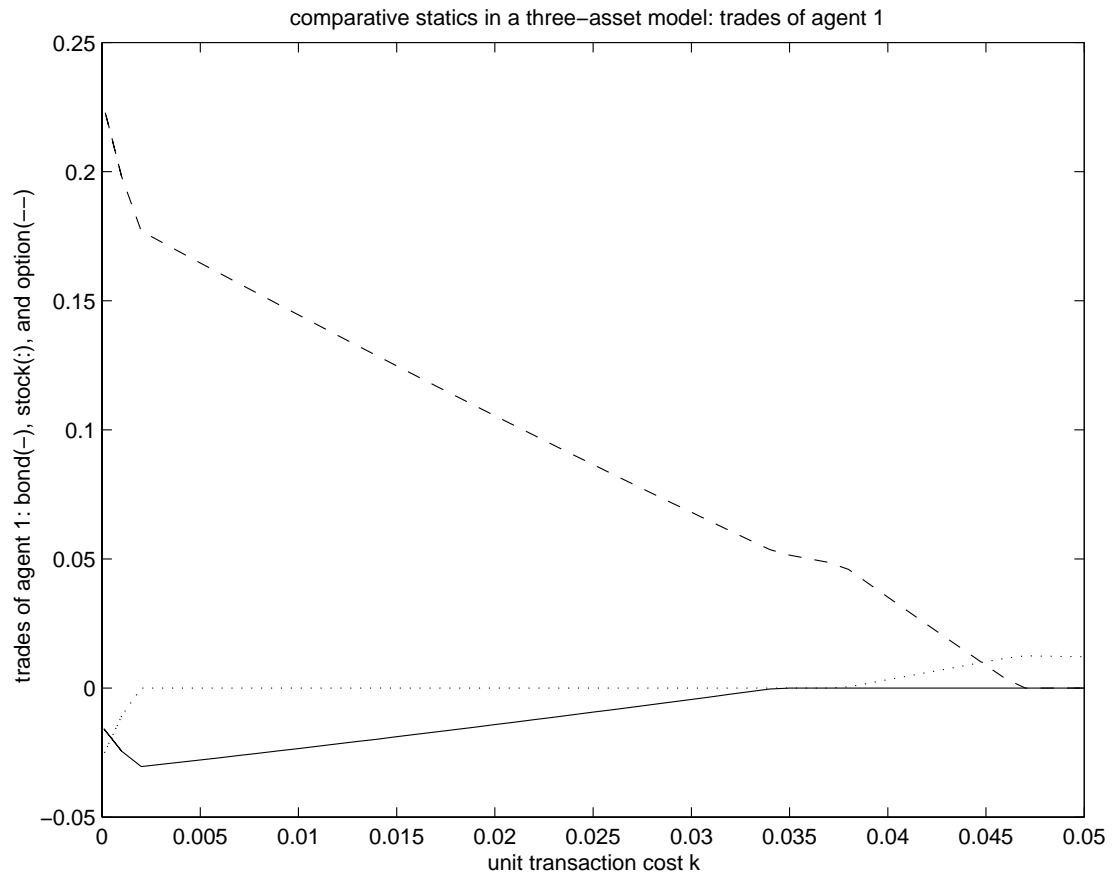

Figure 9: Comparative statics: equilibrium trades of agent 1. 


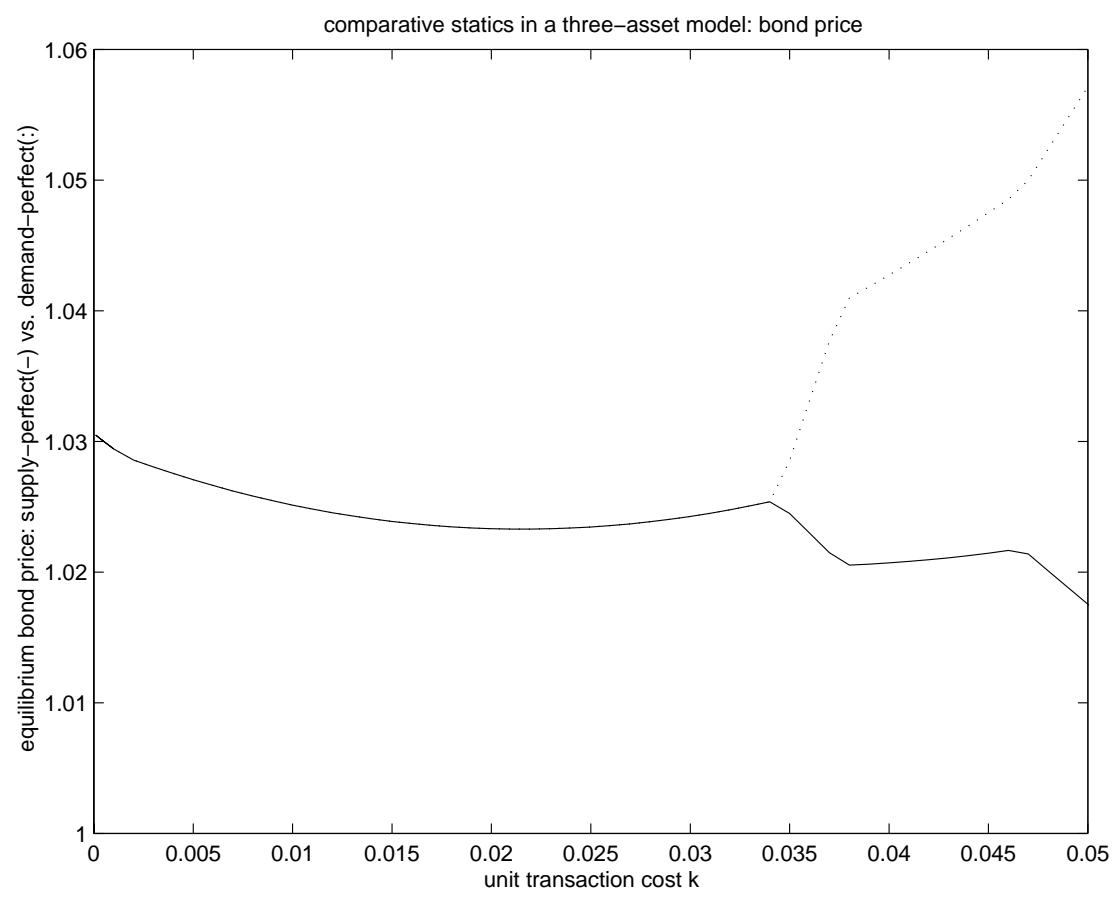

Figure 10: Comparative statics: equilibrium bond price. 


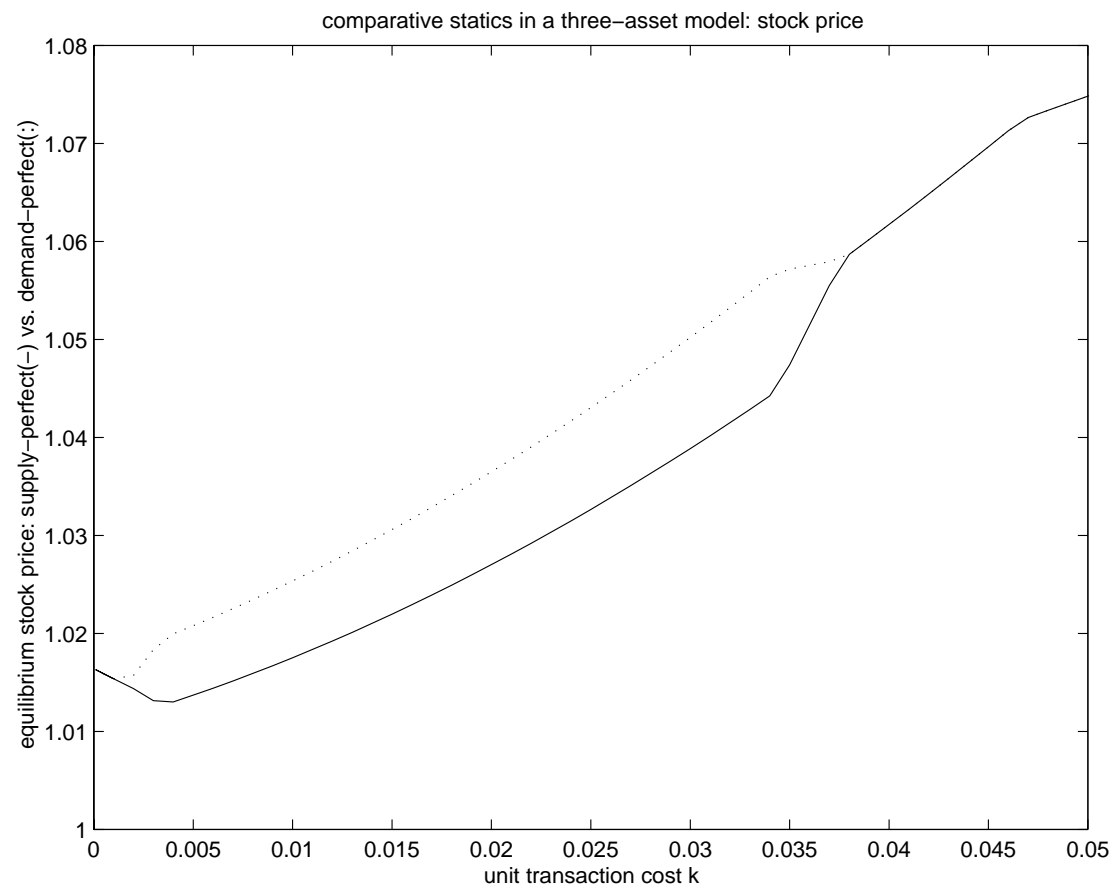

FIGURE 11: Comparative statics: equilibrium stock price. 


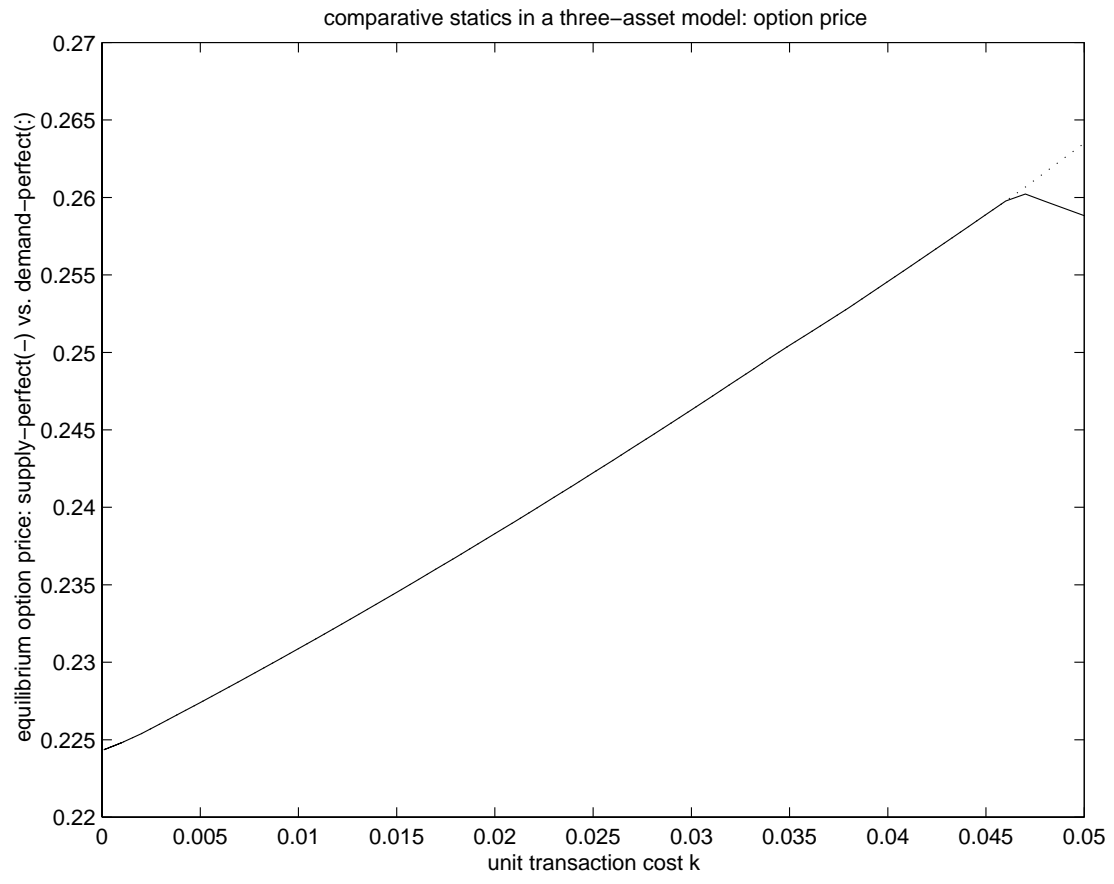

FIgURE 12: Comparative statics: equilibrium option price. 